\title{
Quantum fluctuations around low-dimensional topological defects
}

\author{
Juan Mateos Guilarte ${ }^{* \dagger}$ \\ Departamento de Fisica Fundamental and IUFFyM, Universidad de Salamanca, SPAIN \\ E-mail: guilartedusaL.es
}

\section{A. Alonso Izquierdo}

Departamento de Matematica Aplicada and IUFFyM, Universidad de Salamanca, SPAIN

E-mail: alonsoizausal.es

\section{W. Garcia Fuertes}

Departamento de Fisica, Universidad de Oviedo, SPAIN

E-mail: wifredoduniovi.es

\section{M. de la Torre Mayado}

Departamento de Fisica Fundamental and IUFFyM, Universidad de Salamanca, SPAIN

E-mail: marinadusal.es

\section{J. Senosiain}

Departamento de Matematicas, Universidad de Salamanca, SPAIN

E-mail: lidiazabaldusal.es

In these Lectures a method is described to analyze the effect of quantum fluctuations on topological defect backgrounds up to the one-loop level. The method is based on the spectral heat kernel/zeta function regularization procedure, and it is first applied to various types of kinks arising in several deformed linear and non-linear sigma models with different numbers of scalar fields. In the second part, the same conceptual framework is constructed for the topological solitons of the planar semilocal Abelian Higgs model, built from a doublet of complex scalar fields and one $U(1)$ gauge field.

5th International School on Field Theory and Gravitation,

April 20 - 242009

CuiabÃ a city, Brazil

\footnotetext{
* Speaker.

${ }^{\dagger}$ We are grateful to the chairman and co-chairman of the School, Professors Carlos Pinheiro and Gentil O. Pires, for inviting us to lecture at such a stimulating event. We also appreciate the work of the local organizers Professors Alberto Arruda and Harold Blas, who have succeeded in achieving, together with the main organizers and other collaborators, a remarkable School of Physics. Concerning the material presented in these Lectures we are grateful above all to P. van Nieuwenhuizen for solid, illuminating, and constructive criticism of our work in this field, communicated to us by electronic mail. We thank also e-mail correspondences and/or oral conversations with A. Rebhan, R. Wimmer, M. Bordag, D. Vassilevich and H. Gies on this subject. Finally, we acknowledge the Spanish Ministerio de Educacion y Ciencia and Junta de Castilla y Leon for partial financial support under grants FIS2006-09417, GR224, and SA034A08. J. M. G. thanks the ESF Research Network CASIMIR for providing excellent opportunities for discussion on the Casimir effect and related topics like topological defect fluctuations.
} 


\section{Introduction}

The concept of solitary waves was brought to light in 1834 by the Scottish civil engineer Scott Rusell, chasing a single "wave of translation" on a horse along a channel in Edinburgh. Unlike ordinary dispersive waves, these non-linear waves do not fade away and they preserve their shape, size and speed even they undergo weak perturbations. A major step forward in the conceptual understanding of this counterintuitive phenomenon was the discovery of the Korteweg-de Vries equation in 1895 which admits solutions exhibiting the features of solitary waves. Moreover, there are stronger relatives of solitary waves among the solutions of the KdV equation -the solitonswhich even survive collisions amongst themshelves. During the celebrated sixties of the past century, a powerful technique was invented, the inverse scattering method, which allowed the solution of many "integrable" non-linear partial differential equations in the class of the KdV equations. For a recent review on the concept of soliton associated with all these non-linear equations, see, e.g., Reference [四].

Our theme in these Lectures, however, is the analysis of the quantum descendants of these classical non-linear solitary waves/topological defects in one and two spatial dimensions. In 1961, Skyrme [2] discovered that a certain extension of the non-linear sigma model, the so called Skyrme model, has both 3D dispersive and solitary waves among their solutions. Because the model attempted to describe the low-energy hadron phenomenology, and because solitary waves are formed from a heavy classical lump of energy, the idea is natural: upon quantization, dispersive waves become light mesons -pions- and solitary waves give rise to heavy baryons -protons, neutrons-. This bold idea prompted the task of investigating solitary waves in the quantum domain, mainly performed in [3], [四], and [5] as far as our Lectures are concerned. The main examples were reported and the conceptual framework was extraordinarily well clarified in [6]. We insist that there are many more authors who contributed to developing this research topic. Some of them took another approach, and there are many very good reviews in the Proceedings of several Schools, and even important old and modern books. Most of the pertinent bibliography is collected in Reference $[\mathbb{~}]$. Here, we only cite the papers and reviews with an approach close to ours: the $\hbar /$ weak coupling/semiclassical expansion.

The plan of these Lectures is to describe a method for computing one-loop fluctuations in one-dimensional and two-dimensional topological defects based on the heat kernel/zeta function regularization of ultraviolet divergences. The method began in Reference [ [ $]$ ] with the calculation of the one-loop mass shift to the masses of the paradigmatic sine-Gordon and $\lambda \phi_{2}^{4}$ kink as a test. In the same paper, some of us calculated the one-loop mass corrections to other two-scalar field theoretical models with only one field but with insufficient information about the kink fluctuation spectrum to apply the conventional Dashen-Hasslacher-Neveu approach. In References [Q] and [ए]], the same group generalized the method to provide one-loop mass corrections for two-component topological kinks: i.e., the models addressed have two scalar fields and the kinks considered are such that the two components of the scalar field are not zero for the kink solution. The kink fluctuations of such type of kinks are determined by non-diagonal Schrödinger operators and the only possibility for managing the spectral information needed to compute one-loop mass shifts is the heat kernel expansion.

Prior to our work, at the end of the last century, there had been a renaissance in interest about 
the problem of the quantization of classical lumps. The new impetus came from the subtleties arising in the quantization of supersymmetric kinks. Several groups at Stony Brook/Wien, [ए]], [ㅁ] ], Minnesota [[13], and MIT [14] addressed mixed issues in the problem by studying the impact made by using different types of boundary conditions - PBC, Dirichlet, Robin-, regularization methods -energy cutoff, mode number cutoff, high-derivatives-, and/or performed phase shift analyses, in connection with possible modifications due to the quantum effects of the central charge of the SUSY algebra. There are several valuable reviews, of different character and scope, in the literature on these developments: e.g., [ए5], [ए]], and [ए]]].

The stimulus to our work on the quantization of solitons came, however, from the discovery of kinks in theories with two scalar fields living on a infinite line. In this type of model, there are often solitary wave solutions such that the two scalar fields are space-dependent for the kink profiles, see

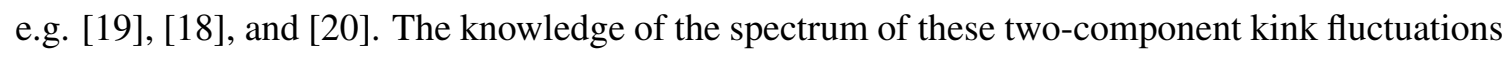
is insufficient to profit from any type of Dashen-Hasslacher-Neveu approach. The only possibility is to use the spectral zeta function obtained from the heat kernel asymptotic (high-temperature) expansion. This framework was precisely chosen in the SUSY kink problem in Reference []ㅐ].

Kinks are one-dimensional topological defects, but extremely interesting two-dimensional extended structures were discovered by Abrikosov in Type II superconductors [D2]. The phenomenological Ginzburg-Landau theory allowed the existence of magnetic flux lines when applied to this type of superconducting materials. Relativistic cousins of Abrikosov strings exist in the Abelian Higgs model and were proposed by Nielsen and Olesen in 1973, see [22] ], as plausible candidates as the basic objects in the early string theory approach to hadron physics. More recently Achucarro and Vachaspati have discovered even more complex two-dimensional topological defects in the so called semilocal Abelian Higgs model, the bosonic sector of electro-weak theory when the weak (Weinberg, mixing) angle is $\frac{\pi}{2}$ [[24]. This model enjoys a symmetry group that is the direct product of two groups: $\mathbb{S U}(2) \otimes \mathbb{U}(1)$. The non-Abelian group $\mathbb{S U}(2)$ engenders a global symmetry whereas the other symmetry generated by the $\mathbb{U}(1)$ factor is local (or gauge). Henceforth, Achucarro and Vachaspati christened the topological solitons of this system as semilocal strings. Given the important rôle that these models play in our present understanding of the Standard model, it is convenient to address the problem of studying the quantum behavior of these two-dimensional solitons, sometimes referred to as ANO vortices or semilocal vortices because the vector (gauge) field of these solutions is purely vorticial (rotational). This task was successfully accomplished in the $\mathscr{N}=2$ SUSY Higgs model independently by Vassilevich, [25], and Rebhan, van Nieuwenhuizen, and Wimmer, [2]].

In the bosonic setting, however, without fermions to cancel a good deal of the bosonic fluctuations, the problem is much more difficult. A good step forward towards this goal was given in Reference [27] in which the authors calculate the energy of the fermionic ANO vortex fluctuations. We profited from our experience with multi-component kinks to compute the one-loop mass shifts of ANO vortices with a quantum of magnetic flux in a purely bosonic setting in [28]. To this end we used the heat kernel/zeta function regularization method, jumping painfully from one to two dimensions. In [2Q] our calculations were extended to superposed vortices up to four quanta of magnetic flux and we attacked the problem of computing one-loop mass shifts to the topological solitons of the generalized Abelian Higgs model in Reference [B]]. We summarized all this material in the Proceedings of QFEXT05 and QFEXT07 published in [30] and [32]. 
One might think that this is a very narrow and highly focused subject. This way of thinking is not completely true, for two reasons. First, knowledge of quantum field theories with topological sectors other than vacuum sectors is not fully settled down, at least at the level of perturbation theory around the ground state. Second, study of the quantum fluctuations around topological defects is a problem in the kinship of very important physical phenomena, such as the cosmological constant problem and the Casimir effect. Vacuum fluctuations (loop graphs) give rise to a non-zero constant term in the Lagrangian of the Standard Model. Coupling of this Lagrangian to gravity means that the constant term is a cosmological constant induced by the quantum fluctuations of the particle fields of an order of magnitude greater than the experimental value of $\sim 10^{60}$. The Casimir effect is an even closer physical phenomenon. Vacuum fluctuations also play a central rôle. Here, the idea is to sum the effect of the vacuum fluctuations in the presence of some set up -parallel plates, cylinders, spheres- measured with respect to the vacuum. The outcome is the appearance of physical forces on the plates emerging from the vacuum.

Our goal in this report is to present an analysis of the quantum corrections to the mass of topological defects developed in different one-dimensional and two-dimensional systems in the set of References cited above in a manner as unified as possible. The contribution is divided into two separate parts. In the first, we deal with one-dimensional relativistic scalar fields. We explain the problem, the method of solution chosen, and the derivation of a compact formula for one-loop kink mass shifts in a multi-parametric family of deformed linear $\mathbb{O}(N)$-sigma models. The $\lambda \phi^{4}$ model is a member of this family for only one scalar field: $N=1$. Our approach is tested in this prototypical case, and detailed computations are offered. We also describe the results achieved in another member of this family with $N=2$ scalar fields having degenerate families of topological kinks. The first part ends with an analysis of the kink one-loop mass shifts of the topological kinks in the massive non-linear $\mathbb{S}^{2}$-sigma model studied in Reference [33]. Again the model is embedded in the family of linear $\mathbb{O}(N)$-sigma models, taking the formal $\lambda \rightarrow \infty$ in the case of $N=3$ scalar fields, a process that ends with a non-linear field theory. Following [34], we compute the mass shift using the Cahill-Comtet-Glauber formula, see [5].

Part two is devoted to understanding the quantum fluctuations of semilocal strings and NielsenOlesen vortices. The action of the semilocal Abelian Higgs model is considered when the spacetime is the $(2+1)$-dimensional Minkowski $\mathbb{R}^{2,1}$. The mix of local and global symmetries, the Higgs mechanism in the 't Hooft renormalizable gauge, and the Feynman rules are discussed. We then go on to study the very rich moduli space of topological soliton solutions, all of them having vorticial vector fields. Numerical solutions of the first-order field equations arising at the critical point between Type I and Type II superconductivity are calculated in the case of circular symmetry. These planar solitons become strings seen from $(3+1)$ dimensions. The next task is an analysis of the semilocal self-dual vortex fluctuations and the subsequent vortex Casimir and mass renormalization energies. These ultraviolet divergent quantities are regularized via the spectral zeta function of the second-order fluctuation operators as in kink cases. Unlike kink cases, the pertinent differentials are $6 \times 6$ - Matrix second-order PD operators. In the background gauge there are fluctuations corresponding to the Higgs field, a real Higgs ghost field, a complex massless scalar field, and the two polarizations of the massive vector field. There are also fluctuations of the Faddeev-Popov ghost field that restore unitarity by compensation of the real Higgs ghost field fluctuations. The heat kernel expansion allows us the calculation of the one-loop vortex mass 
shift in terms of Seeley coefficients and incomplete Euler Gamma functions. Finally, we provide numerical results that suggest the breakdown of the classical degeneracy of the semi-local vortices in favour of the embedded Nielsen-Olesen vortices.

\section{Scalar field models in a line}

In this first part we report the procedure of computing the one-loop mass shifts for onedimensional topological defects developed in [[8], [Q] , and [미]. Ultraviolet divergences are regularized using heat kernel/zeta function methods - comprehensive reviews of these techniques are [B]], [B6], [B]]- and the models considered belong to (1+1)-dimensional scalar field theory. We shall always deal with topological kinks, but in a particular model of $N=2$ scalar fields we must struggle with the problem of studying fluctuations of a degenerate-in-energy continuous family of kinks.

\subsection{Deformed linear $\mathbb{O}(N)$-sigma models}

We shall focus on a multi-parametric family of deformed linear $\mathbb{O}(N)$-sigma models. The target (isospin, internal, $\cdots$ ) space is $\mathbb{R}^{N}$. Let $\chi_{a}, a=1,2, \cdots, N$, denote the coordinates of a point in $\mathbb{R}^{N}$. The (multi-component) scalar fields are maps from the $(1+1)$-dimensional Minkowski space to the target space: $\chi_{a}\left(y^{\mu}\right) \in \operatorname{Maps}\left(\mathbb{R}^{1,1}, \mathbb{R}^{N}\right)$. Here $y_{\mu}, \quad \mu=0,1$, denote the coordinates of a point in the Minkowski space-time $\mathbb{R}^{1,1}$. We shall use the following conventions for the metric and volume element:

$$
\begin{aligned}
& y^{\mu} y_{\mu}=y_{0}^{2}-y_{1}^{2}=g^{\mu v} y_{\mu} y_{v} \quad, \quad g^{\mu v}=g_{\mu v}=\operatorname{diag}[1,-1] \\
& d y^{2}=d y_{0} d y_{1} \quad, \quad \frac{\partial \chi_{a}}{\partial y^{\mu}} \cdot \frac{\partial \chi_{a}}{\partial y_{\mu}}=\frac{\partial \chi_{a}}{\partial y_{0}} \frac{\partial \chi_{a}}{\partial y_{0}}-\frac{\partial \chi_{a}}{\partial y_{1}} \frac{\partial \chi_{a}}{\partial y_{1}}
\end{aligned}
$$

The action governing the dynamics of the deformed linear $\mathbb{O}(N)$-sigma model is:

$$
\begin{aligned}
S\left[\chi_{1}, \chi_{2}, \cdots, \chi_{N}\right]= & \frac{1}{2} \int d y^{2}\left\{\sum_{a=1}^{N} \frac{\partial \chi_{a}}{\partial y^{\mu}} \cdot \frac{\partial \chi_{a}}{\partial y_{\mu}}-\frac{\lambda}{2}\left(\sum_{a=1}^{N} \chi_{a} \chi_{a}-\frac{m^{2}}{\lambda}\right)^{2}\right. \\
- & \left.\sum_{a} \sum_{b} \lambda_{a b} \chi_{a}^{2} \chi_{b}^{2}-\sum_{a=1}^{N} m_{a}^{2} \chi_{a} \chi_{a}\right\}
\end{aligned}
$$

We choose a system of units where the speed of light is $c=1$, but we keep $\hbar$ explicit because we shall work in the framework of $\hbar$-expansion. In this system of units the dimension of $\hbar$ is mass $\times$ length, $[\hbar]=M L$, whereas the dimension of the fields and parameters are:

$$
\left[\chi_{a}\right]=M^{\frac{1}{2}} L^{\frac{1}{2}} \quad, \quad[\lambda]=\left[\lambda_{a b}\right]=M^{-1} L^{-3} \quad, \quad[m]=\left[m_{a}\right]=L^{-1} \quad .
$$

Defining non-dimensional coordinates, fields, and coupling constants

$$
x^{\mu}=\frac{m}{\sqrt{2}} y^{\mu} \quad, \quad \phi_{a}\left(x^{\mu}\right)=\frac{\sqrt{\lambda}}{m} \chi_{a}\left(y^{\mu}\right) \quad, \quad \sigma_{a b}=\frac{\lambda_{a b}}{\lambda} \quad, \quad \sigma_{a}^{2}=\frac{m_{a}^{2}}{m^{2}}
$$


the action reads:

$$
\begin{aligned}
& S\left[\phi_{1}, \phi_{2}, \cdots, \phi_{N}\right]=\frac{m^{2}}{\lambda} \int d x^{2}\left\{\frac{1}{2} \sum_{a=1}^{N} \frac{\partial \phi_{a}}{\partial x^{\mu}} \cdot \frac{\partial \phi_{a}}{\partial x_{\mu}}-V\left(\phi_{a} ; \sigma_{a b}, \sigma_{a}^{2}\right)\right\}
\end{aligned}
$$

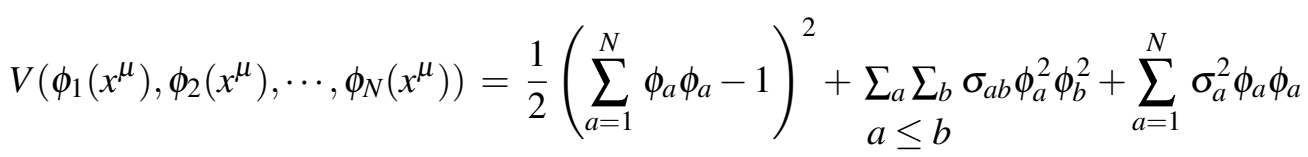

Besides the usual $m$ parameter, which sets the length scale of the system, and the $\lambda$, which sets the strength of the isotropic quartic couplings, there are $\frac{N(N+1)}{2}$ non-isotropic quartic couplings $\sigma_{a b}$ and $N \sigma_{a}^{2}$ parameters giving possible quadratic anisotropies ${ }^{1}$. The rationale behind the choice of this family of models is as follows: the set of parameters $\sigma_{a b}=\sigma_{a}^{2}=0, \forall a, b$ corresponds to the linear $\mathbb{O}(N)$-sigma model. In this case, there are $N-1$ Goldstone bosons, owing to the spontaneous symmetry breaking of the global $\mathbb{O}(N)$ symmetry. Goldstone bosons do not exist in $(1+1)$ dimensions, see [38]. Even if we were to start from the linear $\mathbb{O}(N)$-sigma model, the $(1+1)$-dimensional infrared asymptotics of these massless fields would generate anisotropic quartic and/or quadratic terms, such that no global $\mathbb{O}(N)$ symmetry (or any of its continuous subgroups) would remain. Note that no cubic or linear terms in the fields are allowed because the discrete subgroup $\mathbb{Z}_{2}^{N}$ of $\mathbb{O}(N)$ generated by the internal reflections $\phi_{a} \rightarrow-\phi_{a}, \forall a$, would be explicitly broken.

\subsection{Vacuum fluctuations}

In the parameter range

$$
1>\sigma_{a}^{2}>-\infty, \quad \sigma_{a b}>\max \left(\frac{1-\sigma_{a}^{2}}{1-\sigma_{b}^{2}}\left(1+2 \sigma_{b b}\right), \frac{1-\sigma_{b}^{2}}{1-\sigma_{a}^{2}}\left(1+2 \sigma_{a a}\right)\right)>0, a \neq b, \quad 1+2 \sigma_{a a}>0
$$

the constant configurations

$$
\left(\phi_{1}^{V^{(a)}}=0, \cdots, \phi_{a}^{V^{(a)}}= \pm \sqrt{\frac{1-\sigma_{a}^{2}}{1+2 \sigma_{a a}}}, \cdots, \phi_{N}^{V^{(a)}}=0\right), \quad a=1,2, \cdots, N
$$

are stable solutions of the time-independent and static field equations

$$
\frac{\partial V}{\partial \phi_{a}}=2 \phi_{a}\left[\sum_{b=1}^{N} \phi_{b} \phi_{b}+\sum_{a \leq b} \sigma_{a b} \phi_{b}^{2}+\sigma_{a}^{2}-1\right]=0
$$

These non-zero constant solutions are thus the $2 N$ classical minima of the system. In the quantum domain only the absolute minima

$$
V\left(\phi_{c}^{V^{(c)}}\right)=\frac{\sigma_{c}^{2}\left(2-\sigma_{c}^{2}\right)+2 \sigma_{c c}}{2\left(1+2 \sigma_{c c}\right)} \quad, \quad S^{(0)}\left[\phi_{a}^{V^{(c)}}\right]=\frac{m^{2}}{\lambda} \int d^{2} x V\left(0, \cdots, \pm \sqrt{\frac{1-\sigma_{c}^{2}}{1+2 \sigma_{c c}}}, \cdots, 0\right)
$$

are the true vacua. Tunnel effects triggered by bounces turn the relative minima $V\left(\phi_{a}^{V^{(a)}}\right)>V\left(\phi_{c}^{V^{(c)}}\right)$ into false vacua [40]. The ground states are built from at least one pair of minima (more than a pair of absolute minima could exist) and the $\mathbb{Z}_{2}^{N}$ symmetry is broken spontaneously (at least) to $\mathbb{Z}_{2}^{N-1}$.

\footnotetext{
${ }^{1} \mathrm{~A}$ warning: despite the notation, the possibility that some $\sigma_{a}^{2}$ might be negative will not be ruled out.
} 
Let us denote space-time coordinates in the form $x_{0}=t, x_{1}=x$ and let us consider small fluctuations $\phi_{a}(t, x)=\phi_{a}^{V^{(c)}}+\eta_{a}(t, x)$ around a true vacuum. The action at the quadratic order is

$$
S^{(2)}\left[\phi_{a}^{V^{(c)}} ; \eta_{1}, \cdots, \eta_{N}\right]=\frac{m^{2}}{2 \lambda} \int d x^{2} \sum_{a=1}^{N}\left[\frac{\partial \eta_{a}}{\partial t} \frac{\partial \eta_{a}}{\partial t}-\eta_{a}\left(-\frac{\partial^{2}}{\partial x^{2}}+\mu_{a}^{2}\right) \eta_{a}\right]+\mathscr{O}\left(\eta^{3}\right)
$$

where

$$
\mu_{a}^{2}=\left.\frac{\partial^{2} V}{\partial \phi_{a}^{2}}\right|_{V^{(c)}}=2\left(\frac{1-\sigma_{c}^{2}}{1+2 \sigma_{c c}}\left(1+\sigma_{a c}\right)-\left(1-\sigma_{a}^{2}\right)\right), a \neq c \quad, \quad \mu_{c}^{2}=\left.\frac{\partial^{2} V}{\partial \phi_{c}^{2}}\right|_{V^{(c)}}=4\left(1-\sigma_{c}^{2}\right)
$$

are the particle masses.

The normal modes of these system with a infinite number of degrees of freedom are determined in terms of the eigenfunctions of the differential operator:

$$
K_{0}=\left(\begin{array}{cccc}
-\frac{d^{2}}{d x^{2}}+\mu_{1}^{2} & \ldots & \ldots & 0 \\
0 & -\frac{d^{2}}{d x^{2}}+\mu_{2}^{2} & \ldots & 0 \\
\vdots & \vdots & \ddots & \vdots \\
0 & \ldots & \ldots & -\frac{d^{2}}{d x^{2}}+\mu_{N}^{2}
\end{array}\right) \quad, \quad f_{n}^{a}(x)=\frac{1}{\sqrt{l}} e^{i k_{n} x}
$$

To avoid problems with the continuous spectrum, we choose a 1D "box" of very large but finite length $l=\frac{m L}{\sqrt{2}}$, and we impose periodic boundary conditions: $f^{a}(x+l)=f^{a}(x) . K_{0}$ therefore acts on the Hilbert space $L^{2}=\bigoplus_{a=1}^{N} L_{a}^{2}\left(\mathbb{S}^{1}\right)$ and the eigenvalues

$$
K_{0} f_{n}^{a}(x)=\omega_{a}^{2}\left(k_{n}\right) f_{n}^{a}(x) \quad, \quad \omega_{a}^{2}\left(k_{n}\right)=k_{n}^{2}+\mu_{a}^{2} \quad, \quad k_{n}=\frac{2 \pi}{l} n \quad, \quad n \in \mathbb{Z}
$$

are obtained from wave numbers labeled by the integers.

Classically the system is tantamount to a infinite numerable set of uncoupled oscillators with frequencies given by the eigenvalues of $K_{0}$ that become quantum oscillators upon canonical quantization. The free quantum Hamilton

$$
\hat{H}^{(2)}=\frac{\hbar m}{\sqrt{2}} \sum_{a=1}^{N} \sum_{n \in \mathbb{Z}} \omega_{a}\left(k_{n}\right)\left(\hat{b}_{a}^{\dagger}\left(k_{n}\right) \hat{b}_{a}\left(k_{n}\right)+\frac{1}{2}\right)
$$

is given in terms of the creation and annihilation operators, $\left[\hat{b}_{a}^{\dagger}\left(k_{n}\right), \hat{b}_{c}\left(k_{m}\right)\right]=\delta_{a c} \delta_{m n}$ : the quantum disguise of the Fourier coefficients. Note that $\hat{H}^{(2)}$ is proportional to $\hbar$. In general, the operator $\hat{H}^{(2 j)}$ coming from the $2-j$ th-order fluctuation term in the expansion of the classical action is proportional to $\hbar^{j}$. Therefore, result (2.3]) is obtained in the first-order (one-loop) of the $\hbar$-expansion (loop expansion).

The vacuum state, annihilated by all the destruction operators, is a coherent state, and is an eigen-state of the field operator:

$$
\hat{b}_{a}\left(k_{n}\right)|V ; 0\rangle=0, \forall a, \forall k_{n} \quad, \quad \hat{\phi}_{a}|V ; 0\rangle=0, a \neq c, \quad \hat{\phi}_{c}|V ; 0\rangle=\sqrt{\frac{1-\sigma_{c}^{2}}{1+2 \sigma_{c c}}}|V ; 0\rangle
$$

It is clearly a ground state of the quantum system with energy:

$$
\left\langle 0 ; V\left|\hat{H}^{(2)}\right| V ; 0\right\rangle=\frac{\hbar m}{2 \sqrt{2}} \operatorname{Tr}_{L^{2}} K_{0}^{\frac{1}{2}} \quad .
$$




\subsubsection{Spectral zeta function regularization, the $K_{0}$-heat equation kernel, and the $K_{0}$-heat trace}

We usually measure the energy of any state in QFT with respect to the vacuum or ground state, or, equivalently, we set $\hat{H}^{(2)}|V ; 0\rangle=\frac{\hbar m}{2 \sqrt{2}} \operatorname{Tr}_{L^{2}} K_{0}^{\frac{1}{2}}|V ; 0\rangle$ as the zero-energy level. Important physical phenomena such as the Casimir effect or the cosmological constant problem have taught us that we must be cautious about using this calibration. In particular, in our system there are other topological sectors and it is convenient not to set the energy of the ground state in the vacuum sector to zero a priori in order to allow a comparison with the energy of the ground state in the kink sector.

The problem is that $\frac{\hbar m}{2 \sqrt{2}} \operatorname{Tr}_{L^{2}} K_{0}^{\frac{1}{2}}$ is a divergent quantity that, one way or another must be regularized. Several regularization methods have been proposed in the literature: cut-offs in the energy

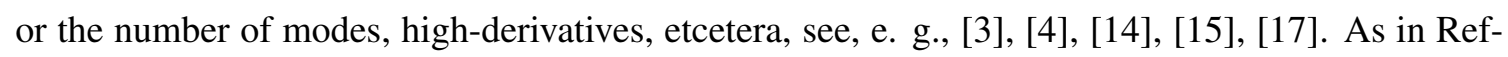
erence [ [2]], however, we shall regularize the vacuum energy using the zeta regularization method. Instead of computing the $L^{2}$-trace of the square root of the $K_{0}$ operator, we calculate the spectral zeta function -the $-s$-complex power of the $K_{0}$ operator:

$$
\zeta_{K_{0}}(s)=\operatorname{Tr}_{L^{2}} K_{0}^{-s}=\sum_{a=1}^{N} \sum_{n=-\infty}^{\infty} \frac{1}{\left(\frac{4 \pi^{2}}{l^{2}} n^{2}+\mu_{a}^{2}\right)^{s}}=\sum_{a=1}^{N} E\left(s, \mu_{a}^{2} \mid \frac{4 \pi^{2}}{l^{2}}\right), \quad s \in \mathbb{C} \quad .
$$

The series in (2.4) are convergent only if $\operatorname{Re} s>\frac{1}{2}$ although conventionally they are analytically continued to the whole $s$-complex plane to find the Epstein zeta functions, all of which are meromorphic functions of $s$, see [B] $]$. The central idea of the zeta function regularization method is to assign to the divergent vacuum energy the finite value

$$
\left\langle 0 ; V\left|\left(\hat{H}^{(2)}\right)^{-s}\right| V ; 0\right\rangle=2^{s-1} \hbar \mu\left(\frac{\mu^{2}}{m^{2}}\right)^{s} \zeta_{K_{0}}(s)
$$

at a regular point $s \in \mathbb{C}$ of $\zeta_{K_{0}}(s) . \mu$ is a parameter of dimension $L^{-1}$ necessary to keep track of the right dimensions.

The analysis of the associated $K_{0}$-heat equation kernel

$$
\left(\frac{\partial}{\partial \beta}+K_{0}\right) K_{K_{0}}(x, y ; \beta)=0 \quad, \quad K_{K_{0}}(x, y ; 0)=\delta(x-y)
$$

will help us to unveil the structure of $\zeta_{K_{0}}(s)$ as a meromorphic function. Here $\beta=\frac{\hbar m}{k_{B} T}$ is a nondimensional inverse temperature because the dimension of the Boltzmann constant in our system of units is $\left[k_{B}\right]=M L$. In terms of the eigenfunctions and eigenvalues of $K_{0}$, one can express the heat kernel in the form:

$$
\operatorname{tr} K_{K_{0}}(x, y ; \beta)=\frac{1}{l} \sum_{a=1}^{N} e^{-\beta \mu_{a}^{2}} \sum_{n \in \mathbb{Z}} e^{\frac{2 \pi}{l} i n(x-y)} e^{-\beta \frac{4 \pi^{2}}{l^{2}} n^{2}}=\frac{1}{l} \Theta\left[\begin{array}{l}
0 \\
0
\end{array}\right]\left(\frac{x-y}{l} \mid i \frac{4 \pi}{l^{2}} \beta\right) \sum_{a=1}^{N} e^{-\beta \mu_{a}^{2}}
$$

The notation used for the Jacobi Theta function, see e.g. [30], is:

$$
\Theta\left[\begin{array}{l}
a \\
b
\end{array}\right](z \mid \tau)=\sum_{n \in \mathbb{Z}} e^{\left.2 \pi i(n+a)(z+b)+\frac{(n+a)^{2}}{2} \tau\right)} \quad ; \quad a, b=0, \frac{1}{2}, \quad z \in \mathbb{C} \quad, \quad \tau \in \mathbb{C}, \operatorname{Im} \tau>0
$$


The "modular" transformation $\tau=i \frac{4 \pi}{l^{2}} \beta \rightarrow-\frac{1}{\tau}=i \frac{l^{2}}{4 \pi \beta}$ allows us to write the heat kernel in the form:

$$
\operatorname{tr} K_{K_{0}}(x, y ; \beta)=\frac{e^{-\frac{(x-y)^{2}}{4 \beta}}}{\sqrt{4 \pi \beta}} \cdot \Theta\left[\begin{array}{l}
0 \\
0
\end{array}\right]\left(-i \frac{l(x-y)}{\beta} \mid i \frac{l^{2}}{4 \pi \beta}\right) \cdot \sum_{a=1}^{N} e^{-\beta \mu_{a}^{2}}
$$

because the Jacobi Theta function is a modular form of weight $\frac{1}{2}$ (alternatively, this equivalence could be derived from the Poisson summation formula). There are thus two ways of writing the $K_{0}$-heat trace (related by the modular transformation):

$$
\begin{gathered}
\operatorname{Tr}_{L^{2}} e^{-\beta K_{0}}=\int_{-\frac{l}{2}}^{\frac{l}{2}} d x \operatorname{tr} K_{K_{0}}(x, x ; \beta)=\Theta\left[\begin{array}{l}
0 \\
0
\end{array}\right]\left(0 \mid i \frac{4 \pi}{l^{2}} \beta\right) \cdot \sum_{a=1}^{N} e^{-\beta \mu_{a}^{2}}=\sum_{a=1}^{N} e^{-\beta \mu_{a}^{2}} e^{-\beta \frac{4 \pi^{2}}{l^{2}} n^{2}} \\
\operatorname{Tr}_{L^{2}} e^{-\beta K_{0}}=\frac{l}{\sqrt{4 \pi \beta}} \cdot \Theta\left[\begin{array}{l}
0 \\
0
\end{array}\right]\left(0 \mid i \frac{l^{2}}{4 \pi \beta}\right) \cdot \sum_{a=1}^{N} e^{-\beta \mu_{a}^{2}}=\frac{l}{\sqrt{4 \pi \beta}} \sum_{a=1}^{N} \sum_{n=-\infty}^{\infty} e^{-\mu_{a}^{2} \beta} e^{-\frac{l^{2}}{4 \beta} n^{2}}
\end{gathered}
$$

The Mellin transform of the first form gives the sum of Epstein functions:

$$
\zeta_{K_{0}}(s)=\frac{1}{\Gamma(s)} \int_{0}^{\infty} d \beta \beta^{s-1} \operatorname{Tr}_{L^{2}} e^{-\beta K_{0}} \sum_{a=1}^{N} E\left(s, \mu_{a}^{2} \mid \frac{4 \pi^{2}}{l^{2}}\right)=\sum_{a=1}^{N} E\left(s, \mu_{a}^{2} \mid \frac{4 \pi^{2}}{l^{2}}\right)
$$

However, Mellin's transform of the Poisson inverted version

$$
\begin{aligned}
\zeta_{K_{0}}(s) & =\frac{1}{\Gamma(s)} \cdot \int_{0}^{\infty} d \beta \beta^{s-1} \sum_{a=1}^{N} e^{-\mu_{a}^{2} \beta}\left(\frac{l}{\sqrt{4 \pi}} \beta^{-\frac{1}{2}} \sum_{n=-\infty}^{\infty} e^{-\frac{l^{2}}{\beta} n^{2}}\right) \\
& =\frac{l}{\sqrt{4 \pi} \Gamma(s)} \cdot \sum_{a=1}^{N}\left(\frac{\Gamma\left(s-\frac{1}{2}\right)}{\mu_{a}^{2 s-1}}+2 \sum_{n \in \mathbb{Z} /\{0\}}\left(\frac{n l}{\mu_{a}}\right)^{s-1 / 2} K_{1 / 2-s}\left(2 \mu_{a} n l\right)\right)
\end{aligned}
$$

identifies the spectral zeta function as a series of modified Bessel functions of the second type, see [B6]. Moreover, formula (2.5]) shows that there are poles of $\zeta_{K_{0}}(s)$ only at the poles of the Euler Gamma function $\Gamma\left(s-\frac{1}{2}\right)$,

$$
s=\frac{1}{2},-\frac{1}{2},-\frac{3}{2},-\frac{5}{2},-\frac{7}{2}, \cdots,-\frac{2 j+1}{2}, \cdots, \quad j \in \mathbb{Z}^{+} \quad
$$

because $K_{1 / 2-s}\left(2 \mu_{a} l n\right)$ are transcendental entire functions, i.e., holomorphic functions of $s$ in $\mathbb{C} / \infty$ with an essential singularity at $s=\infty$. The behavior of the heat trace at high-temperature is determined by the asymptotic formula up to exponentially small terms:

$$
\Theta\left[\begin{array}{l}
0 \\
0
\end{array}\right]\left(0 \mid i \frac{l^{2}}{4 \pi \beta}\right)=\sum_{n \in \mathbb{Z}} e^{-\frac{l^{2}}{4 \beta} n^{2}} \cong_{\beta \rightarrow 0} 1+\mathscr{O}\left(e^{-\frac{c}{\beta}}\right),
$$

which also characterizes the behavior of $\operatorname{Tr}_{L^{2}} e^{-\beta K_{0}}$ for very large $l$. 


\subsection{Kink fluctuations}

\subsubsection{Topological kinks}

Because of the ground- state structure of the deformed linear $\mathbb{O}(N)$-sigma model, there are other static solutions that are not homogeneous. These classical lumps are "one-component topological kinks" (TK1) We shall refer to this type of kinks in this way because: 1) They are topological. Their profiles connect one ground state with another when plotted from $x=-\infty$ to $x=\infty$. 2) They have only one component of the iso-vector field different from zero.

In order to find their profile, one simply looks for solutions of the field equations such that all the field components except the one accommodating the absolute minima are zero: $\phi_{a}^{K^{(c)}}=0, \forall a \neq$ $c$. Under this assumption the classical energy can be written in the Bogomolny form:

$E=\frac{m^{3}}{\sqrt{2} \lambda}\left[\int d x \frac{1}{2}\left(\frac{d \phi_{c}}{d x} \mp \sqrt{2\left(V\left(\phi_{c}\right)-V\left(\phi_{c}^{V^{(c)}}\right)\right)}\right)^{2} \pm \int_{-\phi_{c}^{V^{(c)}}}^{\phi_{c}^{V^{(c)}}} d \phi_{c} \sqrt{2\left(V\left(\phi_{c}\right)-V\left(\phi_{c}^{V^{(c)}}\right)\right)}\right]$.

Therefore, the solutions

$$
\phi_{c}^{K^{(c)}}(x)= \pm \phi_{c}^{V^{(c)}} \tanh \left[\frac{\mu_{c}}{2}\left(x-x_{0}\right)\right]
$$

of the first-order equations

$$
\frac{d \phi_{c}}{d x}= \pm \sqrt{2\left(V\left(\phi_{c}\right)-V\left(\phi_{c}^{V^{(c)}}\right)\right)}
$$

are absolute minima of the energy that solve the static second-order field equations. TK1 kinks are thus space-dependent solutions that interpolate between the two ground states, reached by the kink in different components of the boundary of the spatial line at infinity. They have finite energy,

$$
E\left[\phi_{c}^{K^{(c)}}\right]=2 \frac{m^{3}}{\sqrt{2} \lambda} \int_{-\infty}^{\infty} d x\left[V\left(\phi_{c}^{K^{(c)}}\right)-V\left(\phi_{c}^{V^{(c)}}\right)\right]=\frac{4}{3} \frac{1}{\sqrt{1-\sigma_{c}^{2}}} \frac{m^{3}}{\sqrt{2} \lambda}
$$

and their energy density is spatially distributed. Despite these features these classical lumps are stable because of topological reasons: they belong to topological sectors disconnected from the vacuum sectors in the configuration space.

\subsubsection{Small kink fluctuations}

Our goal is to study these sectors in the quantum domain. We start from the fact that the quantum descendants of TK1 kinks are the ground states in the topological sectors. Small fluctuations around TK1 kinks,

$$
\phi_{a}(t, x)=\phi_{a}^{K^{(c)}}(x)+\eta_{a}(t, x)
$$

are governed by the quadratic action

$$
S^{(2)}\left[\phi_{a}^{K^{(c)}}(x) ; \eta_{1}, \cdots, \eta_{N}\right]=\frac{m^{2}}{2 \lambda} \int d x^{2} \sum_{a=1}^{N}\left[\frac{\partial \eta_{a}}{\partial t} \frac{\partial \eta_{a}}{\partial t}-\eta_{a} K \eta_{a}\right]+\mathscr{O}\left(\eta^{3}\right)
$$


where the second-order fluctuation operator is a diagonal matrix of Pösch-Teller Schrödinger operators :

$$
K=\left(\begin{array}{ccccc}
-\frac{d^{2}}{d x^{2}}+\mu_{1}^{2}-c_{1 c} \cdot \operatorname{sech}^{2}\left(\frac{\mu_{c}}{2} x\right) & \ldots & \ldots & \ldots & 0 \\
\vdots & \ddots & \vdots & \vdots & \vdots \\
0 & \ldots & -\frac{d^{2}}{d x^{2}}+\mu_{c}^{2}-\frac{3}{2} \mu_{c}^{2} \cdot \operatorname{sech}^{2}\left[\frac{\mu_{c}}{2} x\right] \ldots & 0 \\
\vdots & \vdots & \vdots & \ddots & \vdots \\
0 & \ldots & \ldots & \ldots-\frac{d^{2}}{d x^{2}}+\mu_{N}^{2}-c_{N c} \cdot \operatorname{sech}^{2}\left[\frac{\mu_{c}}{2} x\right]
\end{array}\right) .
$$

The bottom of the wells with respect to the thresholds $\mu_{a}^{2}$ are respectively: $-\frac{3}{2} \mu_{c}^{2}$ and $-c_{a c}=$ $-2\left(1+\sigma_{a c}\right)\left(\phi_{c}^{V^{(c)}}\right)^{2}$.

Like the vacuum fluctuations, the normal modes of kink fluctuations are obtained from the eigenfunctions of the $K$ operator: $K_{a a} f_{n}^{a}(x)=\varepsilon_{a}^{2}(n) f_{n}^{a}(x)$. We impose periodic boundary conditions $f^{a}(x+l)=f^{a}(x)$ to escape the problems of the continuous spectrum, and $K$ also acts on: $L^{2}=$ $\bigoplus_{a=1}^{N} L_{a}^{2}\left(\mathbb{S}^{1}\right)$

The spectrum is slightly different for the operator acting on fluctuations along the TK1 kink orbit:

$$
K_{c c}=-\frac{d^{2}}{d x^{2}}+\mu_{c}^{2}-\frac{3}{2} \mu_{c}^{2} \cdot \operatorname{sech}^{2}\left[\frac{\mu_{c}}{2} x\right]
$$

which is summarized in the next Table.

\begin{tabular}{l|c}
\hline Eigenvalues & Eigenfunctions \\
\hline$\varepsilon_{c}^{2}(0)=0$ & $f_{0}^{c}(x)=\operatorname{sech}^{2}\left[\frac{\mu_{c}}{2} x\right]$ \\
$\varepsilon_{c}^{2}(3)=\frac{3}{4} \mu_{c}^{2}$ & $f_{3}^{c}(x)=\sinh \left[\frac{\mu_{c}}{2} x\right] \operatorname{sech}^{2}\left[\frac{\mu_{c}}{2} x\right]$ \\
$\varepsilon_{c}^{2}(k)=k^{2}+\mu_{c}^{2}$ & $f_{k}^{c}(x)=e^{i k x}\left(3 \tanh ^{2}\left[\frac{\mu_{c}}{2} x\right]-1-\frac{6}{\mu_{c}} i k \tanh \left[\frac{\mu_{c}}{2} x\right]-\frac{4}{\mu_{c}^{2}} k^{2}\right)$ \\
\hline
\end{tabular}

Table 1: Spectrum for the Hessian operator acting on fluctuations along the TK1 kink orbit

There are two bound states, one of zero eigenvalue due to the breaking of spatial translation invariance by the kink, and scattering states with thresholds at $\varepsilon_{c}^{2}(0)=\mu_{c}^{2}$. From the scattering eigenfunctions one reads the phase shifts:

$$
f_{k}^{c}(x) \stackrel{x \rightarrow \pm \infty}{\cong} e^{i\left(k x \pm \frac{1}{2} \delta_{c}(k)\right)} \quad \Rightarrow \quad \delta_{c}(k)=-2 \arctan \frac{3 \mu_{c} k}{\mu_{c}^{2}-2 k^{2}}
$$

The periodic boundary conditions select the even ground states and force the momenta to satisfy a transcendent equation:

$$
k l+\delta_{c}(k)=2 \pi n, \quad, \quad k_{n}-\frac{2 \pi}{l} n=\frac{2}{l} \cdot \arctan \frac{3 \mu_{c} k_{n}}{\mu_{c}^{2}-2 k_{n}^{2}} \quad, \quad n \in \mathbb{Z} \quad .
$$

Although the solutions form an infinite discrete set, they can only be identified graphically. The physicist's loophole is to work with very large $l$ such that the information about the continuous 
spectrum is codified in the spectral density:

$$
\rho_{K_{c c}}(k)=\frac{1}{2 \pi}\left(l+\frac{d \delta_{c}(k)}{d k}\right)=-2 \mu_{c}\left(\frac{1}{k^{2}+\mu_{c}^{2}}+\frac{2}{4 k^{2}+\mu_{c}^{2}}\right) \quad .
$$

The operators acting in the orthogonal directions to the orbit, $a \neq c$, are of the type:

$$
K_{a a}=-\frac{d^{2}}{d x^{2}}+\mu_{a}^{2}-c_{a c} \cdot \operatorname{sech}^{2}\left[\frac{\mu_{c}}{2} x\right] \quad, \quad c_{a c}=2\left(1-\sigma_{c}^{2}\right) \frac{1+\sigma_{a c}}{1+2 \sigma_{c c}},
$$

again of Pösch-Teller type, but a with non-null reflection coefficient in general. To describe the

\begin{tabular}{|c|c|}
\hline Eigenvalues & Eigenfunctions \\
\hline $\begin{aligned} \varepsilon_{a}^{2}(j) & =\left(\mu_{a}^{2}-\frac{\mu_{c}^{2}\left(A-\left(j+\frac{1}{2}\right)\right)^{2}}{4}\right) \\
\varepsilon_{a}^{2}(k) & =k^{2}+\mu_{a}^{2}\end{aligned}$ & 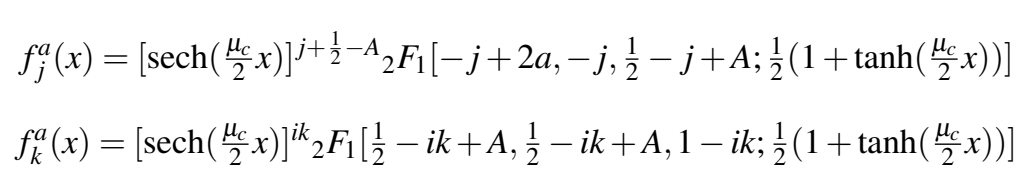 \\
\hline
\end{tabular}
spectrum we define the parameter $A=\sqrt{\frac{1+\sigma_{a c}}{1+2 \sigma_{c c}}+\frac{1}{4}}$.

Table 2: Spectrum for the Hessian operator acting on orthogonal fluctuations to the TK1 kink orbit

The integers $j=0,1,2, \cdots ; I\left[A-\frac{1}{2}\right]$ label the eigenvalues and eigenfunctions of the integer part of the $A+\frac{1}{2}$ bound states. There are also scattering eigenfunctions. Both bound states and scattering eigenfunctions are Gauss hypergeometric functions [42] times some power of the hyperbolic secant.

From this information, we obtain the reflection and transmission coefficients, as well as the phase shifts, the transcendent equation for momenta complying with periodic boundary conditions (PBC), and the spectral densities:

$$
\begin{gathered}
T_{a}(k)=\frac{\Gamma\left(\frac{1}{2}-i k+A\right) \Gamma\left(\frac{1}{2}-i k-A\right)}{\Gamma(1-i k) \Gamma(-i k)} \quad, \quad R_{a}(k)=\frac{T_{a}(k) \Gamma(1-i k) \Gamma(i k)}{\Gamma\left(\frac{1}{2}-A\right) \Gamma\left(\frac{1}{2}+A\right)} \\
\delta_{a}(k)=\delta_{a}^{+}(k)+\delta_{a}^{-}(k) \quad ; \quad \delta_{a}^{ \pm}(k)=\frac{1}{4} \arctan \left(\frac{\operatorname{Im}\left(T_{a}(k) \pm R_{a}(k)\right)}{\operatorname{Re}\left(T_{a}(k) \pm R_{a}(k)\right.}\right) \\
k_{n} l+\delta_{a}\left(k_{n}\right)=2 \pi n \quad, \quad \rho_{K_{a a}}(k)=\frac{1}{2 \pi}\left(l+\frac{d \delta_{a}}{d k}(k)\right)
\end{gathered}
$$

\subsubsection{Spectral kink zeta function regularization}

Let us assume, temporarily, that all the eigenvalues orthogonal to the TK1 kink orbit are positive: $\varepsilon_{a}(j)>0, \forall a$. This means that the TK1 kink is isolated in the configuration space and stable. We expand the small kink fluctuations in a basis in $L^{2}$ formed by the eigenfunctions of $K$ :

$$
\begin{aligned}
\eta_{a}(t, x) & =\sqrt{\frac{\lambda \hbar}{m l}}\left\{\sum_{j=0}^{N_{b}(a)} \frac{1}{\sqrt{2 \varepsilon_{a}(j)}}\left(B_{a}(j)^{*} e^{-i \varepsilon_{a}(j) t}+B_{a}(j) e^{i \varepsilon_{a}(j) t}\right) f_{j}^{a}(x)\right. \\
& \left.+\sum_{k_{n}} \frac{1}{\sqrt{2 \varepsilon_{a}\left(k_{n}\right)}}\left(B_{a}\left(k_{n}\right)^{*} e^{-i \varepsilon_{a}\left(k_{n}\right) t} f_{k_{n}}^{* a}(x)+B_{a}\left(k_{n}\right) e^{i \varepsilon_{a}\left(k_{n}^{2}\right) t} f_{k_{n}}^{a}(x)\right)\right\}
\end{aligned}
$$


We denote here the number of even bound states in the direction of component $a$ as $N_{b}(a)$ and remark that the zero-mode eigenfunction in the direction of the kink orbit $c$ does not enter this formula because zero modes only contribute at higher orders in the loop expansion.

Promoting the coefficients of the expansion to the quantum operators

$$
\left[\hat{B}_{a}^{\dagger}\left(k_{n}\right), \hat{B}_{c}\left(k_{m}\right)\right]=\delta_{a c} \delta_{m n} \quad, \quad\left[\hat{B}_{a}^{\dagger}(j), \hat{B}_{c}(l)\right]=\delta_{a c} \delta_{j l}
$$

one obtains the free Hamiltonian in the kink sector, assuming ortho-normality and completeness of the chosen basis:

$$
\hat{H}^{(2)}=\frac{\hbar m}{\sqrt{2}} \sum_{a=1}^{N}\left[\sum_{j=1}^{N_{b_{+}}(a)} \varepsilon_{a}(j)\left(\hat{B}_{a}^{\dagger}(j) \hat{B}_{a}(j)+\frac{1}{2}\right)+\sum_{k_{n}} \varepsilon_{a}\left(k_{n}\right)\left(\hat{B}_{a}^{\dagger}\left(k_{n}\right) \hat{B}_{a}\left(k_{n}\right)+\frac{1}{2}\right)\right]
$$

The ground state in this sector (no kink fluctuation at all) is also a coherent state

$$
\left.\hat{B}_{a}\left(k_{n}\right)|K ; 0\rangle=0, \forall a, \forall k_{n} ; \quad \hat{\phi}_{a}|K ; 0\rangle=0, a \neq c, \quad \hat{\phi}_{c}|K ; 0\rangle= \pm \phi_{c}^{V^{(c)}} \tanh \left[\frac{\mu_{c}}{2}\left(x-x_{0}\right)\right] K ; 0\right\rangle \quad .
$$

The kink ground-state energy is divergent

$$
\left\langle 0 ; K\left|\hat{H}^{(2)}\right| K ; 0\right\rangle=\frac{\hbar m}{2 \sqrt{2}} \operatorname{Tr}_{L^{2}} K^{\frac{1}{2}}
$$

but we shall regularize it by means of the zeta function prescription: let us take the value of

$$
\left\langle 0 ; K\left|\left(\hat{H}^{(2)}\right)^{-s}\right| K ; 0\right\rangle=2^{s-1} \hbar \mu\left(\frac{\mu^{2}}{m^{2}}\right)^{s} \operatorname{Tr}_{L^{2}} K^{-s}=2^{s-1} \hbar \mu\left(\frac{\mu^{2}}{m^{2}}\right)^{s} \zeta_{K}(s)
$$

at a regular point $s \in \mathbb{C}$ of $\zeta_{K}(s)$. The problem is that, since the wave numbers $k_{n}$ are determined by a transcendent equation, there is no way of writing the $\operatorname{Tr}$ as any manageable series. We shall rely on the less rigorous (physicists) formula:

$$
\zeta_{K}(s)=\operatorname{Tr}_{L^{2}} K^{-s}=\sum_{a=1}^{N}\left(\sum_{j=1}^{N_{b}(a)} \frac{1}{\varepsilon_{a}^{s}(j)}+\int_{-\infty}^{\infty} d k \rho_{K_{a a}}(k) \frac{1}{\left(k^{2}+\mu_{a}^{2}\right)^{\frac{s}{2}}}\right)
$$

where in the $l=\infty$ limit all the bound states $N_{b}(a)=N_{b_{+}}(a)+N_{b_{-}}(a)$ must be accounted for and the "sum" over the continuous spectra must be weighted with the appropriate spectral density.

As in the vacuum sector, there is the $K$-heat equation kernel

$$
\left(\frac{\partial}{\partial \beta}+K\right) K_{K}(x, y ; \beta)=0 \quad, \quad K_{K}(x, y ; 0)=\delta(x-y)
$$

solved for very large $l$ by:

$$
\operatorname{tr} K_{K}(x, y ; \beta)=\sum_{a=1}^{N}\left[\sum_{j=1}^{N_{b}(a)}\left(f_{j}^{a}(x)\right)^{*} f_{j}^{a}(y) e^{-\beta \varepsilon_{a}(j)}+\frac{1}{2 \pi} \int_{-\infty}^{\infty} \frac{d k}{N_{a}(k)}\left(f_{k}^{a}(x)\right)^{*} f_{k}^{a}(y) e^{-\beta\left(k^{2}+\mu_{a}^{2}\right)}\right],
$$

where $N_{a}(k)$ is a normalization factor that depends on the wave number. The heat trace reads:

$$
\operatorname{Tr}_{L^{2}} e^{-\beta K}=\int_{-\frac{l}{2}}^{\frac{l}{2}} d x \operatorname{tr} K_{K}(x, x ; \beta)=\sum_{a=1}^{N}\left[\sum_{j=1}^{N_{b}(a)} e^{-\beta \varepsilon_{a}(j)}+\int_{\infty}^{\infty} d k \rho_{K_{a a}}(k) e^{-\beta\left(k^{2}+\mu_{a}^{2}\right)}\right]
$$

which can be used to determine the kink spectral zeta function via the Mellin transform:

$$
\zeta_{K}(s)=\frac{1}{\Gamma(s)} \int_{0}^{\infty} d \beta \operatorname{Tr}_{L^{2}} e^{-\beta K}
$$




\subsubsection{High-temperature expansion of the $K$-heat kernel and truncated zeta functions}

We have met two levels of information concerning the spectra of small fluctuations. The spectrum of vacuum fluctuations is fully known and it is possible to analytically determine the zeta function regularization of the sum over eigenfrequencies in terms of (famous) meromorphic functions. This possibility fails dealing with fluctuations around stable and isolated TK1 kinks, but knowledge of the scattering data, bound state eigenvalues, phase shifts and spectral densities, allows us to obtain formulas for the spectral zeta function in the form of integrals (rather than series).

The situation is less favourable in the following three cases:

1. There exist one or several zero modes but no negative eigenvalues in orthogonal directions to the TK1 orbit: $\varepsilon_{a}(0)=0, \quad a \neq c$. The TK1 kink is stable but degenerated in energy with a $k$-parametric family of $k$-component topological kinks (TKk), also stable. $k$ is the number of non-translational zero modes.

2. There exist one or several negative modes but no zero eigenvalues in directions orthogonal to the TK1 orbit: $\varepsilon_{a}(0)<0, \quad a \neq c$. The TK1 kink is unstable and decay to some stable and isolated two-component topological kink (TK2).

3. There exist one or several zero modes and one or several negative eigenvalues in directions orthogonal to the TK1 orbit. Isolated or degenerated families of TKk stable kinks arise.

The outcome is the same in these three situations when multi-component stable kinks arise: $K$ is a non-diagonal matrix Schrödinger operator and the spectrum is unknown.

To cope with the problem of multi-component topological kinks we write the $K$-heat equation in the form

$$
\left(\frac{\partial}{\partial \beta}+K_{0}-U(x)\right) K_{K}(x, y ; \beta)=0
$$

and look for a solution based on the $K_{0}$-heat kernel:

$$
K_{K}(x, y ; \beta)=C_{K}(x, y ; \beta) K_{K_{0}}(x, y ; \beta) \quad,
$$

where the density $C_{K}(x, y ; \beta)$ satisfies the infinite temperature condition $C_{K}(x, y ; 0)=\mathbb{I}_{N \times N}$ and the transfer equation:

$$
\left(\frac{\partial}{\partial \beta}+\frac{x-y}{\beta} \frac{\partial}{\partial x}-\frac{\partial^{2}}{\partial x^{2}}\right) C_{K_{a b}}(x, y ; \beta)=\sum_{c=1}^{N} U_{a c}(x) C_{K_{c b}}(x, y ; \beta)+\left(\mu_{b}^{2}-\mu_{a}^{2}\right) C_{K_{a b}}(x, y ; \beta)
$$

Then, we seek a power series solution $C_{K_{a b}}(x, y ; \beta)=\sum_{n=0}^{\infty} c_{n}^{a b}(x, y) \beta^{n}$ of (2.6), which is tantamount to the recurrence relations

$$
n c_{n}^{a b}(x, y)+(x-y) \frac{\partial c_{n}^{a b}}{\partial x}(x, y)=\frac{\partial^{2} c_{n-1}^{a b}}{\partial x^{2}}(x, y)+\sum_{c=1}^{N} U_{a c}(x) c_{n-1}^{c b}(x, y)+\left(\mu_{b}^{2}-\mu_{a}^{2}\right) c_{n-1}^{a b}(x, y)
$$


In fact, only the densities at coincident points $x=y$ on the line are needed. We introduce the notation ${ }^{(k)} C_{n}^{a b}(x)=\lim _{x \rightarrow y} \frac{\partial^{k} c_{n}^{a b}}{\partial x^{k}}(x, y)$ to write the recurrence relations between these densities and their derivatives in the abbreviated form

$$
{ }^{(k)} C_{n}^{a b}(x)=\frac{1}{n}\left\{{ }^{(k+2)} C_{n-1}^{a b}(x)+\sum_{c=1}^{N} U_{a c}(x){ }^{(k)} C_{n-1}^{c b}(x)+\left(\mu_{b}^{2}-\mu_{a}^{2}\right)^{(k)} C_{n-1}^{a b}(x)\right\},
$$

to be solved starting from: ${ }^{(k)} C_{0}^{a b}(x)=\delta^{k 0} \delta_{a b}$. The $n$-th density is determined in terms of the $(n-1)$-order coefficients, their second derivatives, and the potential matrix elements $U_{a b}(x)$.

The solution of these recurrence relations is achieved by using a symbolic program run in Mathematica. We list the lower densities up to the third order:

$$
\begin{aligned}
c_{1}^{a b}(x) & =U_{a b}(x) \quad, \quad U_{a b}^{(k)}(x)=\frac{d^{k} U_{a b}}{d x^{k}}(x) \\
c_{2}^{a b}(x) & =\frac{1}{6} U_{a b}^{(2)}(x)+\frac{1}{2} U_{a b}^{2}(x)+\frac{1}{2}\left(\mu_{b}^{2}-\mu_{a}^{2}\right) U_{a b}(x) \\
c_{3}^{a b}(x) & =\frac{1}{60} U_{a b}^{(4)}(x)+\frac{1}{12}\left(\left(U^{(2)} U+U U^{(2)}\right)_{a b}(x)+\left(U^{(1)} U^{(1)}\right)_{a b}(x)\right)+\frac{1}{6} U_{a b}^{3}(x)+ \\
& +\frac{1}{12}\left(\mu_{b}^{2}-\mu_{a}^{2}\right)\left(U_{a b}^{(2)}(x)+2 U_{a b}^{2}(x)\right)+\frac{1}{6}\left(\sum_{c=1}^{N}\left(\mu_{b}^{2}-\mu_{c}^{2}\right) U_{a c}(x) U_{c b}(x)+\left(\mu_{b}^{2}-\mu_{a}^{2}\right) U_{a b}(x)\right) .
\end{aligned}
$$

There is an interesting point about these densities: the diagonal components are the infinite conserved charges of some matrix $\mathrm{KdV}$ equation, see [4] ] Consider the family of differential operators

$$
K(\beta)=-\frac{\partial^{2}}{\partial x^{2}}+\operatorname{diag}\left(\mu_{1}^{2}, \cdots, \mu_{N}^{2}\right)-U(x, \beta),
$$

where the family of potentials solve the matrix $\mathrm{KdV}$ equation:

$$
\frac{\partial U}{\partial \beta}+3\left(U \frac{\partial U}{\partial x}+\frac{\partial U}{\partial x} U\right)+\frac{\partial^{3} U}{\partial x^{3}}=0
$$

The $\beta$-evolution of $K(\beta)$ can be expressed in the Lax pair form

$$
\frac{\partial K}{\partial \beta}+[K, M]=0 \quad, \quad M(\beta)=4 \frac{\partial^{3}}{\partial x^{3}}-3\left(U \frac{\partial}{\partial x}+\frac{\partial}{\partial x} U\right)+B(\beta)
$$

such that it is iso-spectral. The diagonal densities codify the spectrum of $K$. Ergo, $c_{n}^{a a}(x, x) \neq f(\beta)$.

Integration of the densities gives the asymptotic expansion of the $K$-heat trace:

$\operatorname{Tr}_{L^{2}} e^{-\beta K}=\operatorname{tr} \int_{-\infty}^{\infty} d x K_{K}(x, x ; \beta)=\sum_{a=1}^{N} \frac{e^{-\beta \mu_{a}^{2}}}{\sqrt{4 \pi}} \sum_{n=0}^{\infty} c_{n}^{a a}[K] \beta^{n-\frac{1}{2}} \quad, \quad c_{n}^{a a}[K]=\lim _{l \rightarrow \infty} \int_{-\frac{l}{2}}^{\frac{l}{2}} d x c_{n}^{a a}(x)$

\subsubsection{Truncated zeta function}

Finally, we split the Mellin transform

$$
\begin{aligned}
\zeta_{K}^{*}(s) & =\frac{1}{\Gamma(s)} \int_{0}^{\infty} d \beta \beta^{s-1}\left(\operatorname{Tr}_{L^{2}} e^{-\beta K}-n_{0}\right)=\frac{1}{\Gamma(s)} \int_{0}^{b} d \beta \beta^{s-1}\left(\operatorname{Tr}_{L^{2}} e^{-\beta K}-n_{0}\right)+B_{K}(s ; b) \\
& =\frac{1}{\Gamma(s)} \int_{0}^{b} d \beta \beta^{s-1}\left(\sum_{a=1}^{N} \frac{e^{-\beta \mu_{a}^{2}}}{\sqrt{4 \pi}} \sum_{n=0}^{\infty} c_{n}^{a a}[K] \beta^{n-\frac{1}{2}}-n_{0}\right)+\frac{1}{\Gamma(s)} \int_{b}^{\infty} d \beta \beta^{s-1}\left(\operatorname{Tr}_{L^{2}} e^{-\beta K}-n_{0}\right)
\end{aligned}
$$


into meromorphic and entire parts by choosing an upper limit in the $\beta$-integration to be optimized in each particular problem. The number of zero modes, $n_{0}$, must be subtracted. Truncation of the number of terms kept in the meromorphic part

$$
\zeta_{K}^{*}\left(s ; b, N_{0}\right)=\frac{1}{\sqrt{4 \pi} \Gamma(s)}\left(\sum_{a=1}^{N} \sum_{n=0}^{N_{0}} c_{n}^{a a}[K] \frac{\gamma\left[n+s-1 / 2 ; b \mu_{a}^{2}\right]}{\left(b \mu_{a}^{2}\right)^{s+n-1 / 2}}-\frac{n_{0}}{b^{s} s}\right)
$$

provides us with a practical formula for the spectral zeta function to be used in our computations in terms of the incomplete Euler Gamma functions: $\gamma[z ; c]$. The two free parameters $b$ and $N_{0}$ are correlated: the larger $b$, the greater the $N_{0}$ must be chosen to achieve good approximations.

The subtraction of zero modes that we have performed is a very tricky affair. We split the improper integral into two parts:

$$
I\left[n_{0}\right]=I_{1}\left[n_{0}\right]+I_{2}\left[n_{0}\right]=\lim _{\varepsilon \rightarrow 0} \frac{n_{0}}{\Gamma(s)}\left[\int_{0}^{b} d \beta \beta^{s-1} e^{-\varepsilon \beta}+\int_{b}^{\infty} d \beta \beta^{s-1} e^{-\varepsilon \beta}\right] .
$$

We neglect $I_{2}\left[n_{0}\right]$ and regularize the divergent integral $I_{1}\left[n_{0}\right]$ for $\operatorname{Re} s \leq 0$ by assigning to it the value of the analytic continuation of $I^{R}\left[n_{0}\right]=\frac{n_{0}}{b^{s} s \Gamma(s)}$, valid for $\operatorname{Re} s>0$, to the whole complex $s$-plane.

\subsection{One-loop kink mass shift formula}

We shall now perform the renormalizations needed to tame the ultraviolet divergences, part of which we have already regularized using the zeta function regularization method.

\subsubsection{Kink Casimir energy: zero-point renormalization}

The first renormalization consists of subtracting the vacuum zero-point energy. By analogy, we shall call this quantity the kink Casimir energy. In the Casimir effect, the vacuum fluctuations are subtracted from the zero-point energy around some geometrical set-up, e.g., two infinite impenetrable plates.

The regularized kink Casimir energy is:

$$
\triangle E_{K}^{C}(s)=\triangle E_{K}(s)-\triangle E_{K_{0}}(s)=2^{s-1} \hbar\left(\frac{\mu^{2}}{m^{2}}\right)^{s} \mu\left(\zeta_{K}(s)-\zeta_{K_{0}}(s)\right)
$$

The proper kink Casimir energy is the value of this quantity at the physical point $s=-\frac{1}{2}$ :

$$
\triangle E_{K}^{C}=\lim _{s \rightarrow-\frac{1}{2}} \triangle E_{K}^{C}(s)=\frac{\hbar m}{2 \sqrt{2}}\left(\zeta_{K}\left(-\frac{1}{2}\right)-\zeta_{K_{0}}\left(-\frac{1}{2}\right)\right)
$$

Because $s=-\frac{1}{2}$ is a pole of both $\zeta_{K}(s)$ and $\zeta_{K_{0}}(s)$, with different residua in $(1+1)$-dimensional models, the kink Casimir energy is still a divergent quantity.

\subsubsection{Kink energy engendered by mass renormalization}

There are other ultraviolet divergences due to one-loop graphs that are controlled by the normal-ordered of the quantum Hamiltonian. The contraction of two fields at the same point in Minkowski space is the sum of a normal ordered product, all the creation operators placed to the 
left of the annihilation operators, and a divergent integral that in the normalization 1D "box" becomes a familiar divergent series:

$$
\hat{\phi}_{a}^{2}\left(x^{\mu}\right)=: \hat{\phi}_{a}^{2}\left(x^{\mu}\right):+\delta \mu_{a}^{2} \quad, \quad \delta \mu_{a}^{2}=\int \frac{d k}{4 \pi} \frac{1}{\sqrt{k^{2}+\mu_{a}^{2}}}=\frac{1}{2 l} \sum_{n \in \mathbb{Z}} \frac{1}{\left(\frac{4 \pi^{2}}{l^{2}} n^{2}+\mu_{a}^{2}\right)^{1 / 2}}
$$

The quantum Hamiltonian

$$
\hat{\mathscr{H}}\left(\hat{\phi}_{1}\left(x^{\mu}\right), \cdots, \hat{\phi_{N}}\left(x^{\mu}\right)\right)=\sum_{a=1}^{N} \frac{1}{2}\left(\frac{\partial \hat{\phi}_{a}}{\partial t} \frac{\partial \hat{\phi}_{a}}{\partial t}+\frac{\partial \hat{\phi}_{a}}{\partial x} \frac{\partial \hat{\phi}_{a}}{\partial x}\right)+\hat{V}\left(\hat{\phi}_{1}\left(x^{\mu}\right), \cdots, \hat{\phi_{N}}\left(x^{\mu}\right)\right)
$$

is normal-ordered by applying Wick's theorem:

$$
: \hat{\mathscr{H}}:=\hat{\mathscr{H}}+:\left(1-\exp \left[-\hbar \sum_{a=1}^{N} \widehat{\delta \mu_{a}^{2}} \frac{\delta^{2}}{\delta \phi_{a}^{2}}\right] V\right):=\hat{\mathscr{H}}+\hbar \sum_{a=1}^{N} \delta \mu_{a}^{2}: \widehat{\frac{\delta^{2} V}{\delta \phi_{a}^{2}}}:+\mathscr{O}\left(\hbar^{2}\right)
$$

a process that is equivalent, at one-loop order, to adding quadratic counter-terms to the Hamiltonian. Regularizing the divergent coefficients of these counter-terms by means of the spectral zeta function of $K_{0}$

$$
\delta \mu_{a}^{2}(s)=-\frac{1}{l} \frac{\Gamma(s+1)}{\Gamma(s)} \sum_{a=1}^{N} \zeta_{K_{0 a a}}(s+1)
$$

we obtain at a regular point of $\zeta_{K_{0}}(s+1)$ the regularized contribution to the kink energy due to the mass renormalization counter-terms measured with respect to the mass renormalization vacuum energy:

$$
\begin{aligned}
\triangle E_{K}^{R}(s) & =\hbar m \sum_{a=1}^{N} \delta \mu_{a}^{2} \int d x\left(\left\langle 0 ; K\left|: \frac{\widehat{\delta^{2} V}}{\delta \phi_{a}^{2}}:\right| K ; 0\right\rangle-\left\langle 0 ; V\left|: \frac{\widehat{\delta^{2} V}}{\delta \phi_{a}^{2}}:\right| V ; 0\right\rangle\right) \\
& =-\lim _{l \rightarrow \infty} \frac{\hbar m}{2 l}\left(\frac{2 \mu^{2}}{m^{2}}\right)^{s+1 / 2} \frac{\Gamma(s+1)}{\Gamma(s)} \sum_{a=1}^{N} \zeta_{K_{0 a a}}(s+1) \int_{-\frac{l}{2}}^{\frac{l}{2}} d x U_{a a}(x)
\end{aligned}
$$

The expression in the second line of the formula is derived from the fact that normal-ordered products of field operators acting on coherent states select the ordinary product of the field configuration characterizing the state, see [四]. Note also that we have regularized the divergent graphs using $\zeta_{K_{0}}(s+1)$ instead of $\zeta_{K_{0}}(s)$. The reason for this is the convenience of comparing the residua of $\triangle E_{K}^{C}(s)$ and $\triangle E_{K}^{R}(s)$ at the same pole: $s=-\frac{1}{2}$.

Finally, the one-loop mass shift formula is found by seeking the physical value of the $s$ parameter:

$$
\triangle M_{K}=\lim _{s \rightarrow-1 / 2}\left(\triangle E_{K}^{C}(s)+\triangle E_{K}^{R}(s)\right)
$$

\section{$2.5 \mathrm{~N}=1$ : the $\lambda\left(\phi^{4}\right)_{2}$ model}

We briefly describe the standard $\lambda\left(\phi^{4}\right)_{2}$ model. There is only one real scalar field and no deformation parameters. The action is:

$$
S[\phi]=\frac{m^{2}}{2 \lambda} \int d^{2} x\left\{\frac{\partial \phi}{\partial x^{\mu}} \frac{\partial \phi}{\partial x_{\mu}}-\left(\phi^{2}(t, x)-1\right)^{2}\right\} \quad, \quad \sigma_{11}=\sigma_{1}^{2}=0
$$


The very well known vacua and kinks of this model, as well as the differential operators governing the small vacuum and kink fluctuations are written below:

$$
\phi^{V}= \pm 1 \quad, \quad K_{0}=-\frac{d^{2}}{d x^{2}}+4 \quad ; \quad \phi^{K}(x)= \pm \tanh \left(x-x_{0}\right) \quad, \quad K=-\frac{d^{2}}{d x^{2}}+4-\frac{6}{\cosh ^{2} x}
$$
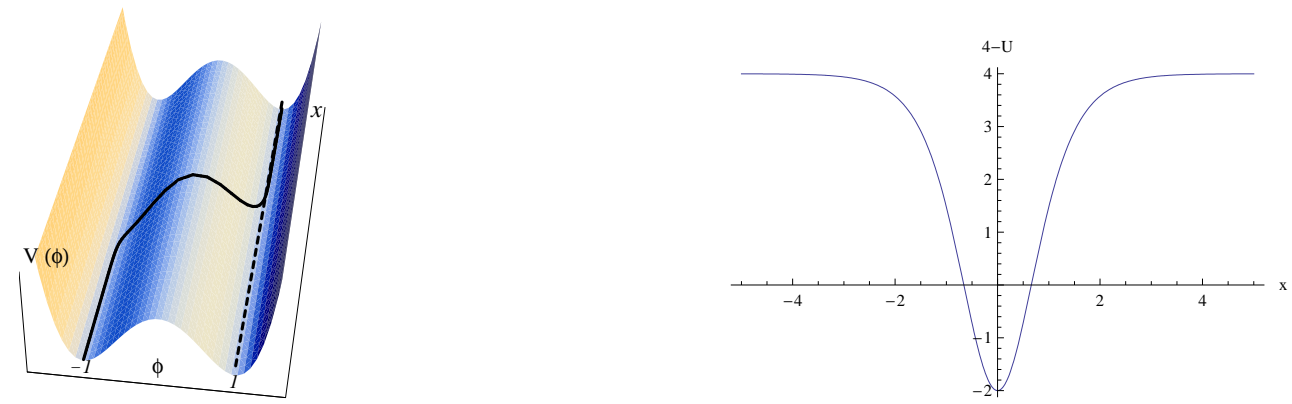

Figure 1: (left) 3D plot of $V$ as a function of both $\phi$ and $x$. The kink is also shown as a black line crossing from one vacuum valley to the other. (right) Graphic of the potential well $4-U(x)$ in the Schrödinger operator $K$.

\subsubsection{Kink spectral heat and zeta functions}

Because the spectrum of $K$ is fully known, both the kink heat function and all the Seeley coefficients can be computed exactly ${ }^{2}$ :

$$
\begin{aligned}
\operatorname{Tr} e^{-\beta K} & =\frac{e^{-4 \beta}}{\sqrt{4 \pi}}\left(\frac{l}{\sqrt{\beta}}+\sqrt{4 \pi}\left(e^{\beta} \operatorname{Erf}[\sqrt{\beta}]+e^{4 \beta} \operatorname{Erf}[2 \sqrt{\beta}]\right)\right) \\
& =\frac{e^{-4 \beta}}{\sqrt{4 \pi}} \sum_{n=0}^{\infty} c_{n}(K) \beta^{n-1 / 2} \quad ; \quad c_{0}(K)=l, \quad c_{n}(K)=\frac{2^{n+1}\left(1+2^{2 n-1}\right)}{(2 n-1) ! !} \quad, \quad n \geq 1
\end{aligned}
$$

where $\operatorname{Erf}[z]$ is the error function. The exact kink zeta function and the truncated theta functions, with the zero mode subtracted, are respectively:

$$
\begin{aligned}
\zeta_{K}^{*}(s) & =\frac{1}{\Gamma(s)} \int_{0}^{\infty} d \beta \beta^{s-1}\left(\operatorname{Tr} e^{-\beta K}-1\right) \\
& =\frac{1}{\sqrt{4 \pi} \Gamma(s)}\left[\frac{l}{4^{s}} \Gamma\left(s-\frac{1}{2}\right)+\left(\frac{2}{3^{s+\frac{1}{2}}} 2_{1}\left[\frac{1}{2}, \frac{1}{2}+s, \frac{3}{2} ;-\frac{1}{3}\right]-\frac{1}{4^{s} s}\right) \Gamma\left(s+\frac{1}{2}\right)\right] \\
\zeta_{K}^{*}\left(s ; b, N_{0}\right) & =\frac{1}{\Gamma(s)} \int_{0}^{b} d \beta \beta^{s-1}\left(\frac{e^{-4 \beta}}{\sqrt{4 \pi}} \sum_{n=0}^{N_{0}} c_{n}(K) \beta^{n-1 / 2}-1\right) \\
& =\frac{1}{\sqrt{4 \pi} \Gamma(s)}\left(l \frac{\gamma\left[s-\frac{1}{2} ; 4 b\right]}{(4 b)^{s-\frac{1}{2}}}+\sum_{n=1}^{N_{0}} \frac{2^{n+1}\left(1+2^{2 n-1}\right)}{(2 n-1) ! !} \frac{\gamma\left[s+n-\frac{1}{2} ; 4 b\right]}{(4 b)^{s+n-\frac{1}{2}}}-\frac{1}{b^{s} s}\right)(2
\end{aligned}
$$

$\gamma[z ; c]$ are incomplete Euler Gamma functions and ${ }_{2} F_{1}[a, b, c ; z]$ are Gauss hypergeometric functions. We follow the notation of [42].

\footnotetext{
${ }^{2}$ In []] , these coefficients are obtained by integration of the densities solving the recurrence relations.
} 


\subsubsection{One-loop $\lambda \phi^{4}$-kink mass shift}

The limit $s \rightarrow-\frac{1}{2}$ is very delicate: it is a pole of $\zeta_{K}(s)$. To take this limit properly we look at the regularized formulae for $s=-\frac{1}{2}+\varepsilon$ and then allow $\varepsilon$ to go to zero:

$$
\begin{aligned}
\Delta E_{K}^{C} & =\frac{\hbar m}{2 \sqrt{2 \pi}} \lim _{\varepsilon \rightarrow 0}\left(\frac{2 \mu^{2}}{m^{2}}\right)^{\varepsilon} \frac{\Gamma(\varepsilon)}{\Gamma\left(-\frac{1}{2}+\varepsilon\right)}\left[\frac{2}{3^{\varepsilon}}{ }_{2} F_{1}\left[\frac{1}{2}, \varepsilon, \frac{3}{2},-\frac{1}{3}\right]-\frac{1}{\left(-\frac{1}{2}+\varepsilon\right) 4^{-\frac{1}{2}+\varepsilon}}\right] \\
& =\frac{\hbar m}{2 \sqrt{2} \pi} \lim _{\varepsilon \rightarrow 0}\left[-\frac{3}{\varepsilon}+2+\ln \frac{3}{4}-3 \ln \frac{2 \mu^{2}}{m^{2}}-{ }_{2} F_{1}^{(0,1,0,0)}\left[\frac{1}{2}, 0, \frac{3}{2},-\frac{1}{3}\right]+o(\varepsilon)\right] \\
& =-\frac{\hbar m}{2 \sqrt{2} \pi} \lim _{\varepsilon \rightarrow 0}\left[\frac{3}{\varepsilon}+3 \ln \frac{2 \mu^{2}}{m^{2}}-\frac{\pi}{\sqrt{3}}\right]
\end{aligned}
$$

where ${ }_{2} F_{1}^{(0,1,0,0)}[a, b, c ; z]$ is the derivative of the hypergeometric function with respect to the second argument. The same strategy is used with $\triangle E_{K}^{R}(s)$ to find:

$$
\begin{aligned}
\Delta E_{K}^{R} & =-\frac{3 \hbar m}{\sqrt{2 \pi}} \lim _{\varepsilon \rightarrow 0}\left(\frac{2 \mu^{2}}{m^{2}}\right)^{\varepsilon} \frac{4^{-\varepsilon} \Gamma(\varepsilon)}{\Gamma\left(-\frac{1}{2}+\varepsilon\right)} \\
& =\frac{3 \hbar m}{2 \sqrt{2} \pi} \lim _{\varepsilon \rightarrow 0}\left[\frac{1}{\varepsilon}+\ln \frac{2 \mu^{2}}{m^{2}}-\ln 4+\left(\psi(1)-\psi\left(-\frac{1}{2}\right)\right)+o(\varepsilon)\right] \\
& =\frac{\hbar m}{2 \sqrt{2} \pi} \lim _{\varepsilon \rightarrow 0}\left[\frac{3}{\varepsilon}+3 \ln \frac{2 \mu^{2}}{m^{2}}-2(2+1)\right],
\end{aligned}
$$

where $\psi(z)$ is the digamma function: i. e., the logarithmic derivative of the Euler Gamma Function. Therefore, the divergences at the pole cancel exactly and we are left with the finite answer:

$$
\triangle M_{K}=\lim _{s \rightarrow-1 / 2}\left(\triangle E_{K}^{C}(s)+\triangle E_{K}^{R}(s)\right)=\left(\frac{1}{2 \sqrt{6}}-\frac{3}{\pi \sqrt{2}}\right) \hbar m=-0.471113 \hbar m
$$

in perfect agreement with the Dashen-Hasslacher-Neveu result in [B]]. We remark that the cancelations above and those implicit in ([2.8) set finite renormalizations, such as the large mass subtraction scheme, by imposing the condition that tadpoles vanish, see Reference [R]].

From the sign of the correction we learn a qualitative fact about the global effect of the scalar boson fluctuations on the kink. The kink energy density is the square of the derivative with respect to $x$ of the kink profile, whereas the kink energy is the area enclosed by this curve. The decrease in kink energy due to kink fluctuations is tantamount to a small decrease in the area. Therefore, the net effect is equivalent to a force from the right and another from the left exerted by the scalar bosons on the kink in such a way that the kink profile shrinks slightly. Exactly as in the ideal Casimir effect with two plates of infinite area.

As a test to prove the quality of the high-temperature approximation, we now write the result for $N_{0}=10$ and $b=1$. See [ [ $]$ ] and [ [ $]$ for full details:

$$
\triangle M_{K} \cong-\left(0.19947+\sum_{n=2}^{10} c_{n}(K) \frac{\gamma[n-1,4]}{8 \pi \sqrt{2} 4^{n-1}}\right) \hbar m=-0.471371 \hbar m
$$

The answer departs from the right result in the four-decimal figure; a reasonably acceptable approximation. There are two elements, however, in formula (2.10) to play with: namely, the length 
$b$ of the integration interval in the Mellin transform and the number $N_{0}$ of terms in the series remaining. Figure 2 shows that these two variables are not independent. For a given value of $N_{0}$, there is an optimum choice of $b$ after which the approximate result (it comes from an asymptotic series) rapidly departs from the exact result. There is no point in enlarging the integration interval. Conversely, taking $b$ longer forces us to increase $N_{0}$ to achieve an acceptable approximation. These remarks are particularly important for light-mass (less than one) particles. The reason is that in such cases we need $b$ to be large because, if not, too much would be neglected in the entire parts.
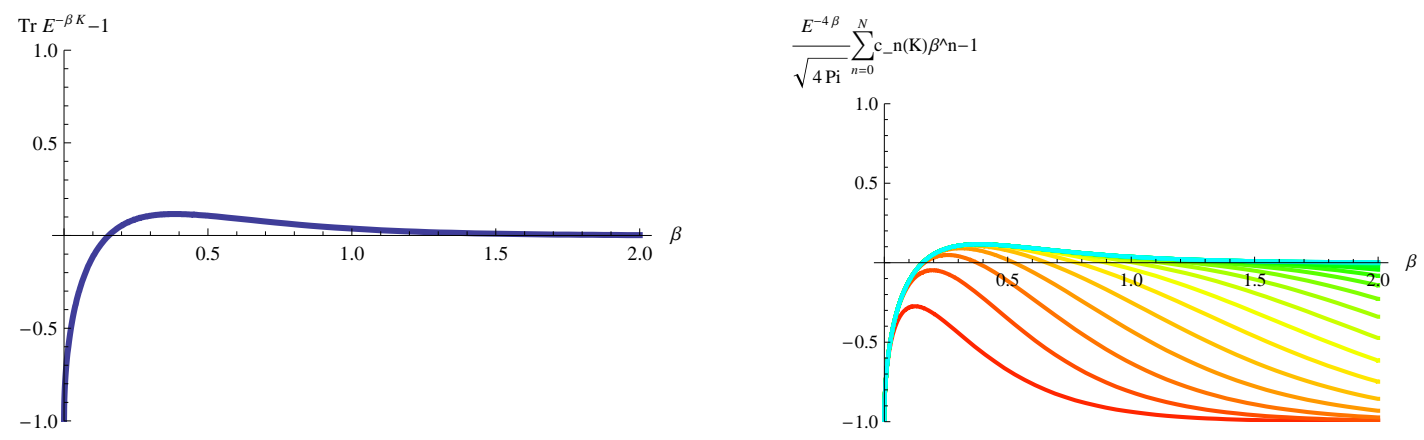

Figure 2: (left) Plot of the exact heat trace as a function of $\beta$ with the zero mode subtracted. (right) Graphics of the approximated formula, keeping $N_{0}=2, N_{0}=3, \cdots, N_{0}=11$ terms.

\subsection{N=2: the BNRT model}

Next, we address a model with two real scalar fields. This field theoretical system can be seen as the bosonic sector of an $\mathscr{N}=1$ supersymmetric Wess-Zumino model of two chiral super-fields dimensionally reduced to $(1+1)$-dimensions (plus a reality condition in the fields), see [ए耳]. Interactions are derived from the simplest polynomial holomorphic super-potential accommodating the second super-field. In fact, the super-potential was discovered independently by Bazeia, Nascimento, Ribeiro, and Toledo (BNRT) [18] directly in the $(1+1)$-dimensional field theoretical model that we shall describe.

The following choice of parameters in the general linear $\mathbb{O}(2)$-sigma model

$$
\sigma_{1}^{2}=0 \quad, \quad \sigma_{2}^{2}=1-\frac{\sigma}{2} \quad, \quad \sigma_{11}=\frac{3}{2} \quad, \quad \sigma_{22}=\frac{\sigma^{2}-1}{2} \quad, \quad 1+\sigma_{12}=2 \sigma(\sigma+1)
$$

leads to the one-parametric family of actions:

$$
\begin{aligned}
S\left[\phi_{1}, \phi_{2}\right] & =\frac{m^{2}}{\lambda} \int d^{2} x\left\{\frac{1}{2}\left(\frac{\partial \phi_{1}}{\partial x^{\mu}} \frac{\partial \phi_{1}}{\partial x_{\mu}}+\frac{\partial \phi_{1}}{\partial x^{\mu}} \frac{\partial \phi_{1}}{\partial x_{\mu}}\right)-\frac{1}{2}\left(\phi_{1}^{2}(t, x)+\phi_{2}^{2}(t, x)-1\right)^{2}\right. \\
& \left.-\frac{3}{2} \phi_{1}^{4}+\frac{1-\sigma^{2}}{2} \phi_{2}^{4}+(1-2 \sigma(\sigma+1)) \phi_{1}^{2} \phi_{2}^{2}+\frac{\sigma-2}{2} \phi_{2}^{2}\right\} .
\end{aligned}
$$

Quartic and quadratic anisotropies are induced by the positive real parameter $\sigma \in \mathbb{R}^{+}$.

\subsubsection{Vacua and TK1 topological kinks}

The four classical minima are degenerated in energy for any $\sigma \in \mathbb{R}^{+}$. Henceforth, all the classical minima are ground states, and there are "two points" in the vacuum moduli space. 
1. Vertical vacua:

$$
\phi_{1}^{V^{(2)}}=0, \phi_{2}^{V^{(2)}}= \pm \frac{1}{\sqrt{2 \sigma}} \quad, \quad V\left(0, \pm \frac{1}{\sqrt{2 \sigma}}\right)=\frac{3}{8} \quad,
$$

such that the particle masses are

$$
\mu_{1}^{2}=2\left(\frac{1-\sigma_{2}^{2}}{1+2 \sigma_{22}}\left(1+\sigma_{12}\right)-\left(1-\sigma_{1}^{2}\right)\right)=2 \sigma \quad, \quad \mu_{2}^{2}=4\left(1-\sigma_{2}^{2}\right)=2 \sigma \quad .
$$

2. Horizontal vacua

$$
\phi_{1}^{V^{(1)}}= \pm \frac{1}{2}, \phi_{2}^{V^{(1)}}=0 \quad, \quad V\left( \pm \frac{1}{2}, 0\right)=\frac{3}{8}
$$

such that the particle masses are

$$
\mu_{1}^{2}=4\left(1-\sigma_{1}^{2}\right)=4 \quad, \quad \mu_{2}^{2}=2\left(\frac{1-\sigma_{1}^{2}}{1+2 \sigma_{11}}\left(1+\sigma_{12}\right)-\left(1-\sigma_{2}^{2}\right)\right)=\sigma^{2}
$$

Therefore, there are also two kinds of one-component topological kinks, see Figure 5.

1. "Vertical" topological kinks:

$$
\phi_{1}^{K}(x)=0 \quad, \quad \phi_{2}^{K}(x)= \pm \frac{1}{\sqrt{2 \sigma}} \tanh \left[\sqrt{\frac{\sigma}{2}}\left(x-x_{0}\right)\right]
$$

The vacuum and kink fluctuation operators are:

$$
K_{0}=\left(\begin{array}{cc}
-\frac{d^{2}}{d x^{2}}+2 \sigma & 0 \\
0 & -\frac{d^{2}}{d x^{2}}+2 \sigma
\end{array}\right) \quad, \quad K=\left(\begin{array}{cc}
-\frac{d^{2}}{d x^{2}}+2 \sigma-\frac{2(1+\sigma)}{\cosh ^{2}\left[\sqrt{\frac{\sigma}{2} x}\right]} & 0 \\
0 & -\frac{d^{2}}{d x^{2}}+2 \sigma-\frac{3 \sigma}{\cosh ^{2}\left[\sqrt{\left.\frac{\sigma}{2} x\right]}\right.}
\end{array}\right) .
$$

2. "Horizontal" topological kinks:

$$
\phi_{1}^{K}(x)= \pm \frac{1}{2} \tanh \left(x-x_{0}\right) \quad, \quad \phi_{2}^{K}(x)=0 \quad .
$$

The vacuum and kink fluctuation operators are:

$$
K_{0}=\left(\begin{array}{cc}
-\frac{d^{2}}{d x^{2}}+4 & 0 \\
0 & -\frac{d^{2}}{d x^{2}}+\sigma^{2}
\end{array}\right) \quad, \quad K=\left(\begin{array}{cc}
-\frac{d^{2}}{d x^{2}}+4-\frac{6}{\cosh ^{2} x} & 0 \\
0 & -\frac{d^{2}}{d x^{2}}+\sigma^{2}-\frac{\sigma(\sigma+1)}{\cosh ^{2} x}
\end{array}\right) .
$$

\subsubsection{Fluctuation spectrum of horizontal TK1 kinks}

We shall not discuss the vertical sector here; the main results can be found in [ए]]. And it is more instructive to explain the horizontal sector. In the kink orbit direction, we meet the Schrödinger operator of the $\lambda \phi^{4}$-kink. There is no need of re-compute again the effect of the fluctuations parallel to the orbit; we simply use the results of the previous model.

Fluctuations orthogonal to the orbit, however, are governed by the Schrödinger operator shown above. We shall focus for a while on the case in which $\sigma=J \in \mathbb{Z}^{+}$is a positive integer. The reason 
for this is that in this discrete set of models transparent (reflection coefficient equal to zero) PöschTeller potentials arise:

$$
K_{22}=-\frac{d^{2}}{d x^{2}}+J^{2}-\frac{J(J+1)}{\cosh ^{2} x}
$$

The previously defined parameter $A$ determining the number of bound states becomes $A=J+\frac{1}{2}$, and the threshold of the continuous spectrum is: $4 \frac{\mu_{a}^{2}}{\mu_{c}^{2}}=J^{2}$. The spectrum of $K_{22}$ is summarized as follows:

1. The eigenvalues and eigenfunctions of the discrete spectrum are:

$$
\begin{aligned}
& \varepsilon_{2}^{2}(j)=(2 J-j) j \quad, \quad j=0,1,2, \cdots, J \\
& f_{0}^{2}(x)=\frac{1}{\cosh ^{J} x} \quad, \quad f_{j}^{2}(x)=\Pi_{r=0}^{j-1}\left(-\frac{d}{d x}+(J-r) \tanh x\right) \frac{1}{\cosh ^{J-j} x} \quad, \quad j \geq 1
\end{aligned}
$$

There are $J$ bound states with eigenvalues below the threshold starting from a zero mode. The highest eigenvalue bound state sits immediately at the threshold of the continuous spectrum. These eigenfunctions are termed half-bound states and they always accompany zero reflection coefficients. In the remarkable array of numbers that follows we have collected the bound state and half-bound state eigenvalues up to $J=10$.

$$
\begin{array}{cccccccccc}
J=1 & J=2 & J=3 & J=4 & J=5 & J=6 & J=7 & J=8 & J=9 & J=10 \\
0 & 0 & 0 & 0 & 0 & 0 & 0 & 0 & 0 & 0 \\
1 & 3 & 5 & 7 & 9 & 11 & 13 & 15 & 17 & 19 \\
& 4 & 8 & 12 & 16 & 20 & 24 & 28 & 32 & 36 \\
& & 9 & 15 & 21 & 27 & 33 & 39 & 45 & 51 \\
& & & 16 & 24 & 32 & 40 & 48 & 56 & 64 \\
& & & & 25 & 35 & 45 & 55 & 65 & 75 \\
& & & & & 36 & 48 & 60 & 72 & 84 \\
& & & & & & 49 & 63 & 77 & 91 \\
& & & & & & & 64 & 80 & 96 \\
& & & & & & & 81 & 99 \\
& & & & & & & & & 100
\end{array}
$$

2. The scattering states are also known and are listed below together with the pase shifts and spectral densities.

$$
\begin{aligned}
& \varepsilon_{2}^{2}(k)=k^{2}+J^{2} \quad, \quad f_{k}^{2}(x)=\Pi_{p=1}^{J}\left(-\frac{d}{d x}+p \tanh x\right) e^{i k x} \\
& \delta_{2}(k)=2 \arctan \left[\frac{\operatorname{Im}\left(\Pi_{p=1}^{J}(p-i k)\right)}{\operatorname{Re}\left(\Pi_{p=1}^{J}(p-i k)\right)}\right] \quad, \quad \rho_{2}(k)=\frac{l}{2 \pi}-\frac{1}{\pi} \sum_{p=1}^{J} \frac{p}{p^{2}+k^{2}}
\end{aligned}
$$

From this information, one derives the exact heat function: 


$$
\begin{aligned}
\operatorname{Tr}_{L^{2}} e^{-\beta K} & =\sum_{j=0}^{J} e^{-\beta(2 J-j)}+\frac{l}{2 \pi} \int_{-\infty}^{\infty} d k e^{-\beta\left(k^{2}+J^{2}\right)}\left[1-\frac{2}{l} \sum_{p=1}^{J} \frac{p}{p^{2}+k^{2}}\right] \\
& =\frac{e^{-\beta N^{2}}}{\sqrt{4 \pi}}\left(\frac{l}{\sqrt{\beta}}+\sqrt{4 \pi} \sum_{p=1}^{J} e^{\beta p^{2}} \operatorname{Erf}[p \sqrt{\beta}]\right)
\end{aligned}
$$

as a sum of error functions. The coefficients of the heat function expansion are also easily calculated:

$$
\begin{aligned}
\operatorname{Tr}_{L^{2}} e^{-\beta K} & =\frac{e^{-\beta J^{2}}}{\sqrt{4 \pi}} \sum_{n=0}^{\infty} c_{n}(K) \beta^{n-\frac{1}{2}} \quad, \quad c_{0}(K)=l \\
c_{n}(K) & =\frac{2^{n+1}}{(2 n-1) ! !} \cdot \sum_{p=1}^{J} p^{2 n-1}=\frac{2^{n+1}}{(2 n-1) ! !}\left(J^{2 n-1}+\frac{B_{2 n}(J)-B_{2 n}}{2 n}\right) \quad, \quad n \geq 1
\end{aligned}
$$

They can be expressed in terms of Bernouilli numbers $B_{2 n}$ and polynomials $B_{2 n}(J)$.

These systems can be useful as patterns for other systems where no analytical information about the spectrum is available and there is no hope of finding the exact heat function. The coefficients of the heat function must be similar for potentials with similar thresholds and areas (number of bound states). Thus, it is important to know the behavior of the heat kernel coefficients in these "integrable" cases. Considering $n$ as a continuous variable $u$, the function

$$
f_{N}(u)=\frac{2^{u+1}}{(2 u-1) ! !} \sum_{p=0}^{J} p^{2 u-1}
$$

is plotted in Figure 3 for three values of $J$. The peaks, as they should be, correspond to integer values $u=n$, and the coefficients pass to zero when $n=12$ for $J=2, n=20$, if $J=3, n=30$ for $J=4$, etcetera. These are the orders at which the double factorial in the denominator dominates the numerator, ensuring the convergence of the series despite the fact that the coefficients grow really large before these orders for $J \geq 4$.
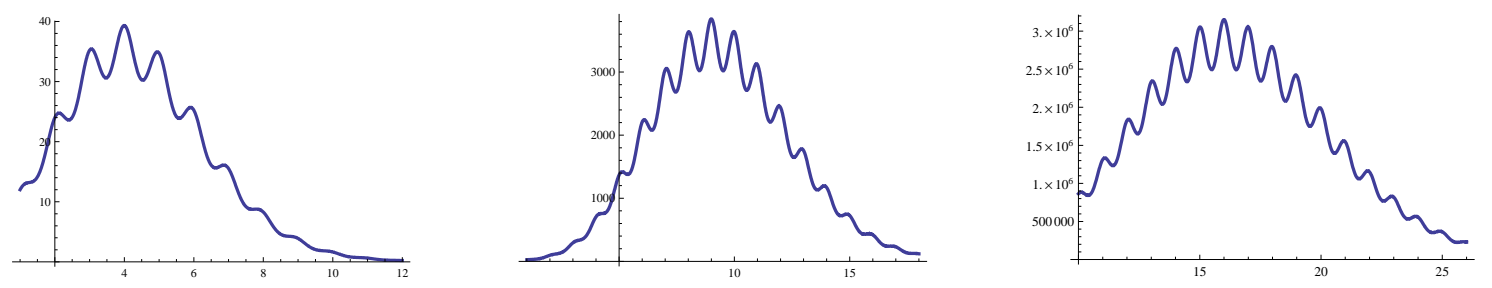

Figure 3: Plots of the function $f_{J}(u)$ : (left) $J=2$ (middle) $J=3$ (right) $J=4$.

\subsubsection{Cancelation of divergences: one-loop mass shifts of horizontal TK1 kinks}

The consequences of the previous analyses can be seen in the magnitude of the one-loop mass shifts. The spectral zeta function

$$
\begin{aligned}
\zeta_{K}^{*}(s) & =\frac{1}{\Gamma(s)} \int_{0}^{\infty} d \beta \beta^{s-1}\left(\operatorname{Tr}_{L^{2}} e^{-\beta K}-1\right) \\
& =\frac{1}{\sqrt{\pi}}\left[\frac{l}{2} \frac{\Gamma\left(s-\frac{1}{2}\right)}{J^{2 s-1} \Gamma(s)}+\left(\sum_{j=1}^{J-1} \frac{2 j}{\left(J^{2}-j^{2}\right)^{s+\frac{1}{2}}} \cdot{ }_{2} F_{1}\left[\frac{1}{2}, \frac{1}{2}+s, \frac{3}{2} ; \frac{-j^{2}}{J^{2}-j^{2}}\right]-\frac{1}{J^{2 s} s}\right) \frac{\Gamma\left(s+\frac{1}{2}\right)}{\Gamma(s)}\right]
\end{aligned}
$$


is essentially a sum of Gauss hypergeometric functions. For instance, in the case $J=3$

$$
\begin{aligned}
\zeta_{K}^{*}(s) & =\frac{1}{\sqrt{\pi}}\left[\frac{l}{2} \frac{\Gamma\left(s-\frac{1}{2}\right)}{3^{2 s-1} \Gamma(s)}\right. \\
& \left.+\left(\frac{2}{8^{s+\frac{1}{2}}} \cdot{ }_{2} F_{1}\left[\frac{1}{2}, \frac{1}{2}+s, \frac{3}{2} ;-\frac{1}{8}\right]+\frac{4}{5^{s+\frac{1}{2}}} \cdot{ }_{2} F_{1}\left[\frac{1}{2}, \frac{1}{2}+s, \frac{3}{2} ;-\frac{4}{5}\right]-\frac{1}{9^{s} s}\right) \frac{\Gamma\left(s+\frac{1}{2}\right)}{\Gamma(s)}\right]
\end{aligned}
$$

one can envisage the general behavior from the array of bound-state energies. The ingredients are the bound-state eigenvalues, the difference in the eigenvalues with respect to the thresholds, and the thresholds themselves (in the subtraction of the zero modes). It is amazing how cleanly the maths capture these fine physical data!

The kink Casimir energy in the direction orthogonal to the orbit for generic $J$ is:

$$
\begin{aligned}
\triangle E_{K}^{C} & =\lim _{s \rightarrow-\frac{1}{2}} 2^{s-1} \hbar\left(\frac{\mu^{2}}{m^{2}}\right)^{s} \mu\left(\zeta_{K_{22}}^{*}(s)-\zeta_{K_{022}}(s)\right) \\
& =\frac{\hbar m}{2 \sqrt{2}} \lim _{\varepsilon \rightarrow 0}\left(\frac{2 \mu^{2}}{m^{2}}\right)^{\varepsilon}\left(\zeta_{K_{22}}^{*}\left(-\frac{1}{2}+\varepsilon\right)-\zeta_{K_{022}}\left(-\frac{1}{2}+\varepsilon\right)\right) \\
& =\frac{\hbar m}{2 \sqrt{2 \pi}} \lim _{\varepsilon \rightarrow 0}\left(\frac{2 \mu^{2}}{m^{2}}\right)^{\varepsilon} \frac{\Gamma(\varepsilon)}{\Gamma\left(-\frac{1}{2}+\varepsilon\right)}\left(\sum_{j=1}^{J-1} \frac{2 j}{\left(J^{2}-j^{2}\right)^{\varepsilon}} \cdot{ }_{2} F_{1}\left[\frac{1}{2}, \varepsilon, \frac{3}{2} ; \frac{-j^{2}}{J^{2}-j^{2}}\right]-\frac{J}{J^{2 \varepsilon}\left(-\frac{1}{2}+\varepsilon\right.}\right) .
\end{aligned}
$$

Simili modo, the kink energy due to the mass renormalization counter-terms of the second particle reads:

$$
\begin{aligned}
\triangle E_{K_{22}}^{R} & =-\frac{\hbar m}{\sqrt{2}} \lim _{l \rightarrow \infty} \frac{1}{2 l} \lim _{s \rightarrow-\frac{1}{2}}\left(\frac{\mu^{2}}{m^{2}}\right)^{s+\frac{1}{2}} \frac{l}{\sqrt{4 \pi}} \cdot \frac{\Gamma\left(s+\frac{1}{2}\right)}{J^{2 s+1} \Gamma(s)} \cdot \int_{-\frac{l}{2}}^{\frac{l}{2}} d x \frac{J(J+1)}{\cosh ^{2} \mathrm{x}} \\
& =-\frac{\hbar m}{2 \sqrt{2 \pi}} \lim _{\varepsilon \rightarrow 0}\left(\frac{2 \mu^{2}}{m^{2}}\right)^{\varepsilon} \frac{J(J+1)}{J^{2 \varepsilon}} \cdot \frac{\Gamma(\varepsilon)}{\Gamma\left(-\frac{1}{2}+\varepsilon\right)} .
\end{aligned}
$$

For instance applying these formulae to the $J=3$ case, we find:

$$
\begin{aligned}
\triangle E_{K_{22}}^{C} & =-\frac{\hbar m}{\sqrt{2} \pi}\left(\lim _{\varepsilon \rightarrow 0} \frac{3}{\varepsilon}+3 \log \frac{2 \mu^{2}}{m^{2}}+\frac{1}{2}\left({ }_{2} F_{1}^{(0,1,0,0)}\left[\frac{1}{2}, 0, \frac{3}{2},-\frac{1}{8}\right]-\log \frac{8}{4}\right)+\right. \\
& \left.+{ }_{2} F_{1}^{(0,1,0,0)}\left[\frac{1}{2}, 0, \frac{3}{2},-\frac{4}{5}\right]-\log \frac{5}{4}-3-\frac{3}{2} \log \frac{9}{4}\right) \\
\triangle E_{K_{22}}^{R} & =\frac{3 \hbar m}{\sqrt{2} \pi}\left(\lim \frac{1}{\varepsilon}+\log \frac{2 \mu^{2}}{m^{2}}-\log \frac{9}{4}-2\right) .
\end{aligned}
$$

The divergent terms, as well as the terms depending on the auxiliary parameter $\mu$ cancel each other exactly, to give:

$$
\triangle M_{K_{22}}=-\frac{\hbar m}{\sqrt{2} \pi}\left[\frac{1}{2}\left({ }_{2} F_{1}^{(0,1,0,0)}\left[\frac{1}{2}, 0, \frac{3}{2},-\frac{1}{8}\right]+\log \frac{9}{8}\right)+{ }_{2} F_{1}^{(0,1,0,0)}\left[\frac{1}{2}, 0, \frac{3}{2},-\frac{4}{5}\right]+\log \frac{9}{5}+3\right] .
$$

Using this result and the analogous one for $J=4$, we obtain the exact answer for the $J=3$, and $J=4$ systems:

$$
\triangle M_{K}^{J=3}=-(0.47113+0.766861) \hbar m \quad, \quad \triangle M_{K}^{J=4}=-(0.47113+1.11725) \hbar m
$$




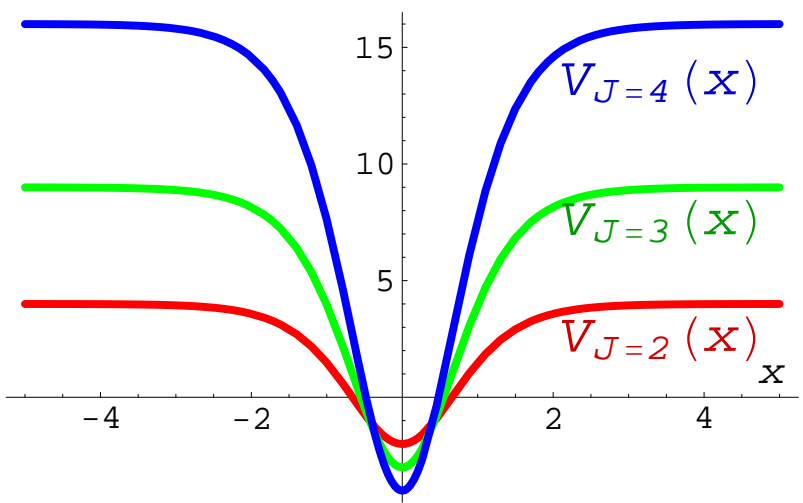

Figure 4: Potential wells for $J=2, J=3$, and $J=4$ plotted together.

The higher the threshold and the broader the area, see Figure 4, the more negative is the correction, because the attraction of the well on the scattered and bound mesons is more intense. The high-temperature asymptotic formula also provides very good approximations to these exact results. We find:

$$
\triangle M_{K}^{J=3}=-(0.47137+0.76675) \hbar m \quad, \quad \triangle M_{K}^{J=4}=-(0.47137+1.11723) \hbar m,
$$

taking $N_{0}=20$ coefficients for $J=3$ and $N_{0}=40$ coefficients if $J=4$ in the asymptotic formulae of $\triangle M_{K_{22}}^{J=3}$ and $\triangle M_{K_{22}}^{J=4}$. The (optimal) truncations in the series have been suggested by the plots in Figure 3. For the same reason, we consider $N_{0}=10$ in the truncated series of $\triangle M_{K_{11}^{J=2}}$.

Even if $\sigma$ is not an integer the one-loop mass shift is still known. The spectrum is more complex but the main differences passing through $J$ are the lack of the (half) bound state, sitting at the threshold, one more bound state and a different spectral density. Because the vacuum half-bound state, (the constant function) is not compensated by a kink half-bound state it must be accounted for in the vacuum spectral function. The half-bound state weight is $\frac{1}{2}$ (hence the name) owing to the one-dimensional Levinson theorem [43], [44]. The jump in the number of bound states miraculously conspires with the change in spectral density to produce a quantum correction that is a continuous function of $\sigma$. Interested readers can find full details about this problem in [ए]]].

\subsubsection{TK2 topological kinks}

The zero-mode fluctuation orthogonal to the kink orbit is a sign of the existence of other topological kinks degenerated in energy with the TK1 kink, which must have the two components of the field different from zero, thus being TK2 kinks. Fortunately, a fair knowledge of the main features and the structure of the TK2 kinks in this model is available. The reason is that the potential energy density can be written in terms of a polynomial "superpotential":

$$
W\left(\phi_{1}, \phi_{2}\right)=2\left(\frac{1}{3} \phi_{1}^{3}-\frac{1}{4} \phi_{1}+\frac{\sigma}{2} \phi_{1} \phi_{2}^{2}\right) \quad, \quad V\left(\phi_{1}, \phi_{2}\right)-\frac{3}{8}=\frac{1}{2} \sum_{a=1}^{2} \frac{\partial W}{\partial \phi_{a}} \cdot \frac{\partial W}{\partial \phi_{a}} \quad .
$$

The energy, arranged à la Bogomolny,

$$
E=\frac{4 m^{3}}{\lambda \sqrt{2}} \int d x \frac{1}{2}\left[\sum_{a=1}^{2}\left(\frac{d \phi_{a}}{d x} \mp \frac{\partial W}{\partial \phi_{a}}\right)\left(\frac{d \phi_{a}}{d x} \mp \frac{\partial W}{\partial \phi_{a}}\right)\right] \pm \frac{4 m^{3}}{\lambda \sqrt{2}} \cdot\left\{W\left(\phi_{a}(+\infty)\right)-W\left(\phi_{a}(-\infty)\right)\right\}
$$


shows that the solutions of the first-order ODE system

$$
\frac{d \phi_{a}}{d x}= \pm \frac{\partial W}{\partial \phi_{a}} \equiv\left\{\begin{array}{l}
\frac{d \phi_{1}}{d x}=(-1)^{\alpha}\left(2 \phi_{1}^{2}+\sigma \phi_{2}^{2}-\frac{1}{2}\right) \\
\frac{d \phi_{2}}{d x}=(-1)^{\beta} 2 \sigma \phi_{1} \phi_{2}
\end{array} \quad, \quad \alpha, \beta=0,1\right.
$$

are absolute minima of the energy.

In [प्व], the kink orbits solving this system of equations were identified for the first time. We found the flow lines of grad $W$ between the top and the bottom of $W$, the kink orbits, in [20] simply by taking the quotient of the first equation by the second in (2.14) to find:

$$
(-1)^{\alpha} \frac{d \phi_{1}}{2 \phi_{1}^{2}+\sigma \phi_{2}^{2}-\frac{1}{2}}+(-1)^{\beta} \frac{d \phi_{2}}{2 \sigma \phi_{1} \phi_{2}}=0
$$

Integration of this first-order equation is achieved using the integrating factor $\left|\phi_{2}\right|^{-\frac{2}{\sigma}}$. The kink orbits are:

$$
\phi_{1}^{2}+\frac{\sigma}{2(1-\sigma)} \phi_{2}^{2}=\frac{1}{4}+\frac{c}{2 \sigma}\left|\phi_{2}\right|^{\frac{2}{\sigma}} \quad, \sigma \neq 1, \quad c \in\left(-\infty, c^{s}=\frac{1}{4} \frac{\sigma}{1-\sigma}(2 \sigma)^{\frac{1+\sigma}{\sigma}}\right)
$$

Note that the integration constant $c$ must be lower than the critical integration function $c^{s}$. The reason for this is easy to understand just by looking at Figure 5. The $c^{s}$ kink orbit joins one horizontal vacuum with the other two vertical vacua. Beyond that value the orbits escape to infinity giving infinite energy to the associated static-field theoretical solutions.
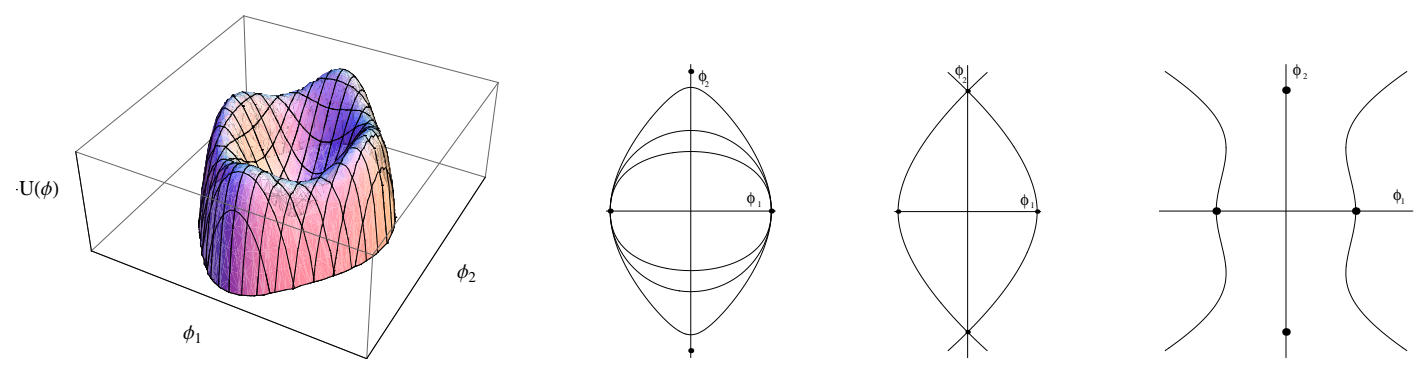

Figure 5: 3D graph of $-V\left(\phi_{1}, \phi_{2}\right)$ as a function of $\phi_{1}$ and $\phi_{2}$ (left) Flow-lines: in the ranges $c \in\left(-\infty, c^{s}\right)$ (middle left), $c=c^{s}$ (middle right), and $c \in\left(c^{s}, \infty\right)$ (right)

The energy of all these TK2 kink orbits is the same:

$$
E\left(\phi_{1}^{K}, \phi_{2}^{K}\right)=\frac{4 m^{3}}{\lambda \sqrt{2}}\left|W\left( \pm \frac{2}{2}, 0\right)-W\left(\mp \frac{1}{2}, 0\right)\right|=\frac{4 m^{3}}{3 \lambda \sqrt{2}}
$$

The zero-energy fluctuations orthogonal to the TK1 kink orbit obeys this fact: there is no cost in energy fluctuating from one kink in this family to another.

For generic $\sigma$, it is not possible go further than this: i.e., knowledge of the TK2 kink orbits and their energy. There are two exceptions, however. If $c=-\infty$, we again find the TK1 kink profile:

$$
\phi_{1}^{1 \mathrm{~K}}(x)=(-1)^{\alpha} \frac{1}{2} \tanh \left(x-x_{0}\right) \quad, \quad \phi_{2}^{1 \mathrm{~K}}(x)=0 \quad, \quad \alpha=0,1 \quad .
$$


For $c=0$, the profiles of the corresponding TK2 kinks are also known analytically:

$\phi_{1}^{2 \mathrm{~K}}(x)=(-1)^{\alpha_{1}} \frac{1}{2} \tanh \left[2(1-\sigma)\left(x-x_{0}\right)\right] \quad, \quad \phi_{2}^{2 \mathrm{~K}}(x)=(-1)^{\alpha_{2}} \sqrt{\frac{1-\sigma}{\sigma}} \operatorname{sech}\left[2(1-\sigma)\left(x-x_{0}\right)\right] \quad$,

where $\alpha_{1}, \alpha_{2}=0,1$. The two kink orbits are half-ellipses.

The generic form of the kink profiles can be inferred from the $\sigma=\frac{1}{2}$ case. Equations (2.14) are separable in parabolic coordinates if $\sigma=\frac{1}{2}$, and we can give the analytic expressions of the kink profiles [[20]:

$$
\begin{aligned}
& \phi_{1}^{2 \mathrm{~K}(d)}\left[x ; x_{0}, d\right]=(-1)^{\alpha_{1}}\left(\frac{1}{2} \frac{\sinh \left(\left(x-x_{0}\right)\right)}{\cosh \left(\left(x-x_{0}\right)\right)+d^{2}}\right) \\
& \phi_{2}^{2 \mathrm{~K}(d)}\left[x ; x_{0}, d\right]=(-1)^{\alpha_{2}}\left(\frac{d}{\sqrt{d^{2}+\cosh \left(\left(x-x_{0}\right)\right)}}\right) \quad, \quad d= \pm \sqrt{\frac{1}{\sqrt{1-4 c}}}
\end{aligned}
$$

There are two integration constants that are the parameters of this TK2 family with a clear physical meaning: $x_{0}$ sets the kink center as in TK1 kinks. The parameter $d$, related to the kink orbit label $c$, can be loosely interpreted as the relative coordinate between two basic kinks.
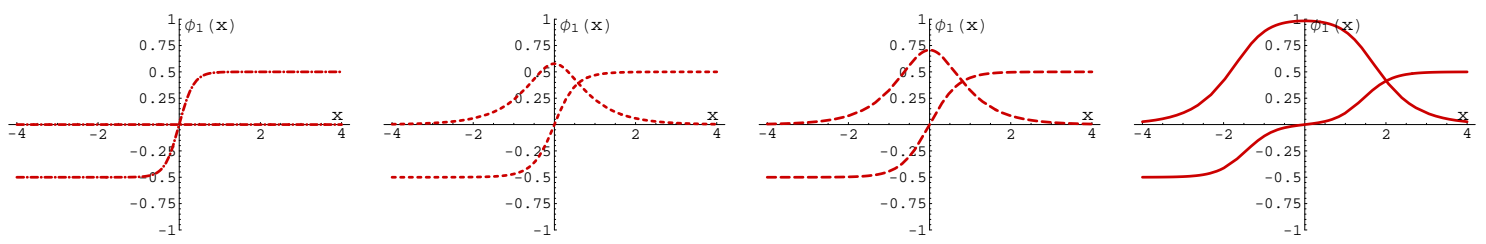

Figure 6: Kink profiles corresponding to: $(a) d=0,(b) d=\sqrt{0.5},(c) d=1$ and $(d) d=\sqrt{30}$.

In Figure 6, the kink profiles for four values of $d$ are shown. If $d=0$ only one component is kink-shaped and non-null. For $d>0$, all the profiles are two-component; one of the components is kink-shaped and the other bell-shaped up to $d=1$. If $d>1$ one component has the shape of two kinks and the other one tends to show two lumps. This Figure is correlated with Figure 7,
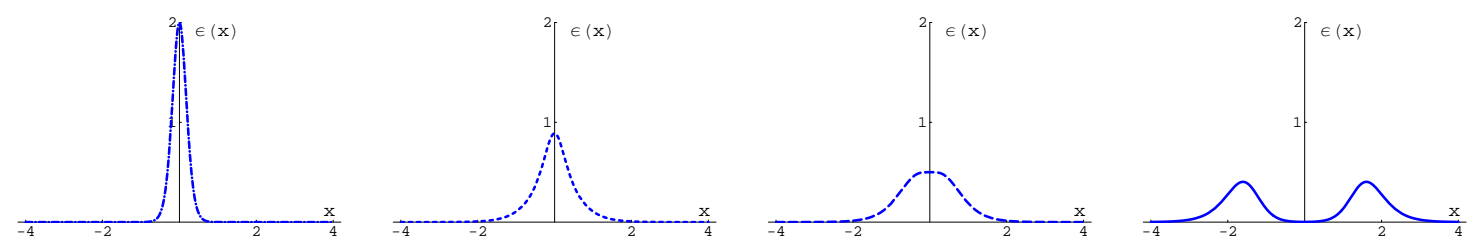

Figure 7: Energy density $\mathscr{E}^{K}[x ; 0, d]$ for $(a) d=0,(b) d=\sqrt{0.5},(c) d=1$ and $(d) d=\sqrt{30}$.

where the energy density is plotted for the same values of $d$. It is possible to prove analytically that the energy density as a function of $x$ has only one maximum if $d \leq 1$ and two maxima if $d>1$. Thus, for $d>1$ this parameter is a relative coordinate between the two peaks, although the distance between maxima is a transcendent function of $d$. The meaning of the peaks is also clear: these values of $d$ correspond to orbits close to the critical orbit where two TK2 kinks joining the horizontal vacuum with different vertical vacua live. These are the basic kinks. All this is explained in detail in Reference [ए]]. 
For generic values of $\sigma$, one must rely on numerical integration methods to find the profiles of the kink solutions. We do this by solving the first-order equations by standard numerical methods with the "initial" conditions:

$$
\phi_{1}(0)=0 \quad, \quad \frac{\sigma}{2(1-\sigma)} \phi_{2}^{2}(0)-\frac{c}{2 \sigma}\left|\phi_{2}(0)\right|^{\frac{2}{\sigma}}=\frac{1}{4}
$$

The reasons for this choice are two fold: (1) For any kink solution, $\phi_{1}(x)$ always has a zero. Translational invariance allows us to set the zero at $x=0$; (2) To ensure that we will find a numerical kink solution, we fix $\phi_{2}(0)$ on a kink orbit for a given value of $\sigma$ and arbitrary choices of $c$.

The numerical method provides us with a succession of points of the kink solution generated by an interpolation polynomial. The plots of the numerical results show that the behavior derived analytically for the kink profiles when $\sigma=\frac{1}{2}$ is generic. For any value of $\sigma$, the kink profiles are composed of two kinks. The parameter $c$ giving the orbit is related to the separation between basic kinks. In some range of $c$, the two basic kinks completely melt into a composite kink. The precise value of $c$ at which this happens depends on the value of $\sigma . \sigma=\frac{1}{2}$ is singled out, because in this case $c=0$ is the value where two kinks fuse into a composite kink or, viceversa, a composite kink splits into two basic kinks.

\subsubsection{TK2 kink small fluctuations}

We consider now small fluctuations of TK2 kinks: $\phi_{a}(x)=\bar{\phi}_{a}(x ; c)+\eta_{a}(x)$, where we denote the TK2 kink profiles in the form $\phi_{a}^{\mathrm{TK} 2(\mathrm{c})}(x)=\bar{\phi}_{a}(x ; c)$. The second-order kink fluctuation operator is a non-diagonal Schrödinger operator:

$$
\begin{aligned}
K(c) & =\left(\begin{array}{cc}
-\frac{d^{2}}{d x^{2}}+4-U_{11}(x ; c) & U_{12}(x ; c) \\
U_{21}(x ; c) & -\frac{d^{2}}{d x^{2}}+4-U_{22}(x ; c)
\end{array}\right) \\
U_{11}(x ; c) & =-\left(24 \bar{\phi}_{1}^{2}(x ; c)+4 \sigma(\sigma+1) \bar{\phi}_{2}^{2}(x ; c)-6\right) \\
U_{12}(x ; c) & =U_{21}(x ; c)=-8 \sigma(\sigma+1) \bar{\phi}_{1}(x ; c) \bar{\phi}_{2}(x ; c) \\
U_{22}(x ; c) & =-\left(4 \sigma(\sigma+1) \bar{\phi}_{1}^{2}(x ; c)+6 \sigma^{2} \bar{\phi}_{2}^{2}(x ; c)-\sigma(\sigma+1)\right)
\end{aligned}
$$

In Figure 8 the potential wells (or barriers) in the diagonal components of $K$ are shown for the integrable case $\sigma=\frac{1}{2}$ for several values of $c$. The correlation with the kink profiles is clear. Therefore, the non-integrable cases have similar wells because the kink profiles obtained numerically are similar to the analytic ones.

The one-loop mass shifts can be computed using the high-temperature formula. We use the first-order equations to express field derivatives as functions of the fields (avoiding problems with the discreteness of space required in numerical methods) and write the Seeley densities. Then, we plug the numerically generated kink profiles into these densities and perform numerical integration over the real line, to finally find the coefficients that enter the formula.

The results are shown in the following Tables for three values of $\sigma: 1.5,2$, and 2.5, and represented in Figure 9. 

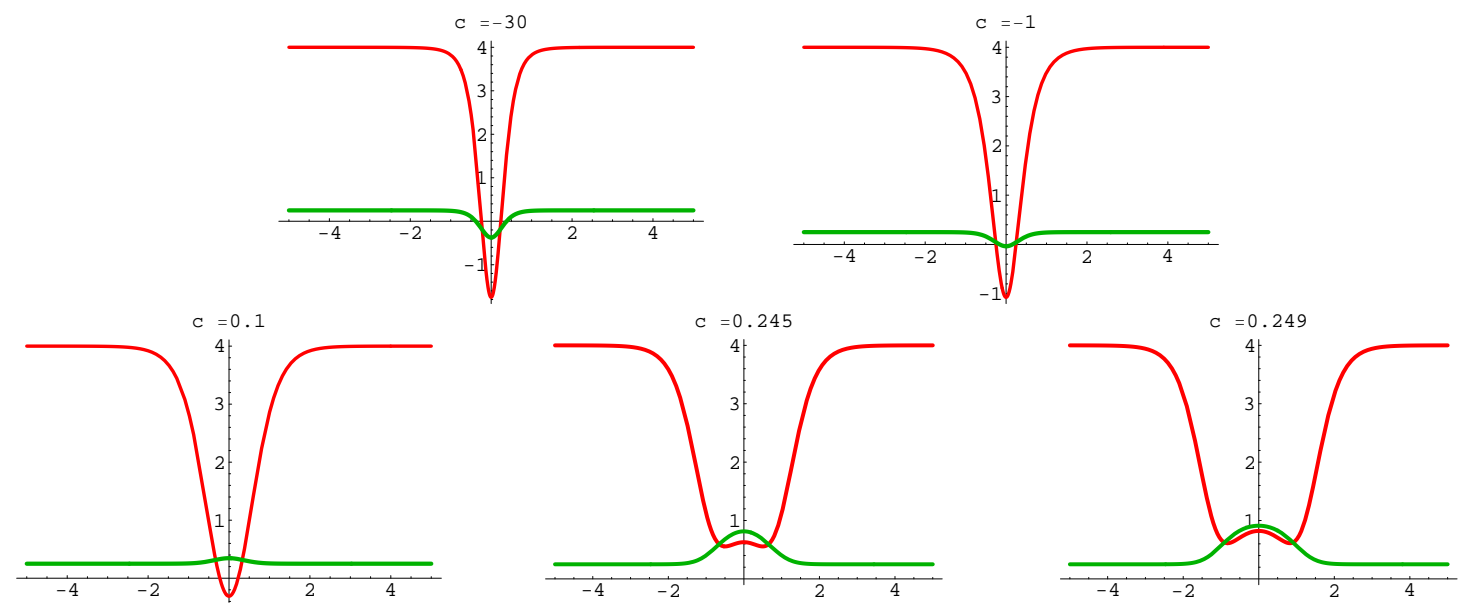

Figure 8: Diagonal components $-U_{11}(x)$ (red) and $-U_{22}(x)$ (green) of the $\sigma=\frac{1}{2}$ potential for $c=-30, c=$ $-1, c=0.1, c=0.245$ and $c=0.249$.

\begin{tabular}{cc}
$\sigma=1.5$ & \\
\hline$c$ & $\Delta M$ \\
\hline-30 & -1.16009 \\
-27.5 & -1.16017 \\
-25 & -1.16128 \\
-22.5 & -1.16042 \\
-20 & -1.16061 \\
-17.5 & -1.16088 \\
-15 & -1.16128 \\
-12.5 & -1.16193 \\
-10 & -1.16313 \\
-7.5 & -1.16597 \\
-5 & -1.18205 \\
-4.6801886 & -1.24345 \\
-4.68018860186678332 & -1.25103 \\
\hline
\end{tabular}

\begin{tabular}{cc}
$\sigma=2.0$ & \\
\hline$c$ & $\Delta M$ \\
\hline-30 & -1.33281 \\
-27.5 & -1.33281 \\
-25 & -1.33281 \\
-22.5 & -1.33281 \\
-20 & -1.33281 \\
-17.5 & -1.33281 \\
-15 & -1.33281 \\
-12.5 & -1.33281 \\
-10 & -1.33281 \\
-7.5 & -1.33281 \\
-5 & -1.33280 \\
-4.001 & -1.33280 \\
-4.00001 & -1.33280 \\
\hline
\end{tabular}

\begin{tabular}{cc}
$\sigma=2.5$ & \\
\hline$c$ & $\Delta M$ \\
\hline-30 & -1.52784 \\
-27.5 & -1.52782 \\
-25 & -1.52780 \\
-22.5 & -1.52778 \\
-20 & -1.52774 \\
-17.5 & -1.52769 \\
-15 & -1.52760 \\
-12.5 & -1.52744 \\
-10 & -1.52711 \\
-7.5 & -1.52626 \\
-5 & -1.52285 \\
-4 & -1.52168 \\
-3.97 & -1.52915 \\
-3.96594571 & -1.55402 \\
-3.96594570565808127 & -1.56127 \\
\hline
\end{tabular}

Table 3: Quantum Mass Corrections to the TK2 family in the BNRT model with $\sigma=1.5,2$, and 2.5

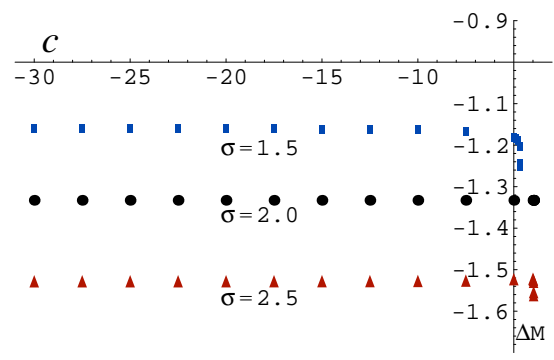

Figure 9: The one-loop mass correction in the cases $\sigma=1.5, \sigma=2.0$, and $\sigma=2.5$

In the $\sigma=1.5$ and $\sigma=2.5$ cases, the pattern is similar. The one-loop correction is equal in the zone where the two basic kinks are fused into a single kink and the degeneracy is not broken at oneloop level. For values of $c$ in the zone where the two basic kinks are split, the one-loop corrections become more and more negative. The classical degeneracy is broken by quantum fluctuations that press the two basic kinks apart from each other. This conclusion could be reached by arguing qualitatively from Figure 8. For small $c$, the potential wells both in the parallel and orthogonal directions to the kink orbit are attractive. After $c=1$ the orthogonal wells start to become weakly 
repulsive. For larger $c$, the parallel wells develop two peaks whereas the orthogonal wells become strongly repulsive. This is the explanation of the repulsion between the two basic kinks and the reason why the less energetic TK2 kink corresponds to maximum separation of the basic kinks, even though the classical energy is independent of the distance between centers. The case $\sigma=2$ is special. The classical degeneracy does not disappear because a simple change of variables shows that this case is equivalent to two independent $\lambda \phi^{4}$ models, such that the one-loop correction is twice the correction of the $\lambda \phi^{4}$ kink.

\section{7 $\mathrm{N}=3$ : the massive non-linear $\mathbb{S}^{2}$-sigma model}

The last scalar field theoretical model that we shall discuss is the massive non-linear $\mathbb{S}^{2}$-sigma model studied in Reference [B3]. This is the formal $\lambda \rightarrow \infty$ limit of the deformed linear $\mathbb{O}(3)$-sigma model, a limit that is only meaningful if the fields satisfy the constraint

$$
\chi_{1}^{2}(t, x)+\chi_{2}^{2}(t, x)+\chi_{3}^{3}(t, x)=R^{2} \quad, \quad R^{2}=\frac{m^{2}}{\lambda}
$$

such that the target space is a $\mathbb{S}^{2}$-sphere of radius $R$. The action becomes

$$
S\left[\chi_{1}, \chi_{2}, \chi_{3}\right]=\frac{1}{2} \int d x^{2}\left\{\sum_{a=1}^{3} \frac{\partial \chi_{a}}{\partial x_{\mu}} \cdot \frac{\partial \chi_{a}}{\partial x^{\mu}}-\alpha_{1}^{2} \chi_{1}^{2}(t, x)-\alpha_{2}^{2} \chi_{2}^{2}(t, x)-\alpha_{3}^{2} \chi_{3}^{2}(t, x)\right\},
$$

where $\alpha_{1}^{2}=2 \sigma_{1}^{2}, \alpha_{2}^{2}=2 \sigma_{2}^{2}, \alpha_{3}^{2}=2 \sigma_{3}^{2}$.

Despite appearances this is a highly non-linear system due to the constraint between the fields. This statement is made evident by solving $\chi_{3}$ in terms of $\chi_{1}$ and $\chi_{2}$. The action becomes:

$$
S=\frac{1}{2} \int d t d x\left\{\partial_{\mu} \chi_{1} \partial^{\mu} \chi_{1}+\partial_{\mu} \chi_{2} \partial^{\mu} \chi_{2}+\frac{\left(\chi_{1} \partial_{\mu} \chi_{1}+\chi_{2} \partial_{\mu} \chi_{2}\right)^{2}}{R^{2}-\chi_{1}^{2}-\chi_{2}^{2}}-\chi_{1}^{2}(t, x)-\sigma^{2} \cdot \chi_{2}^{2}(t, x)\right\}
$$

The vacua are the North and South poles: $\chi_{3}= \pm 1$. The parameter $0<\sigma^{2}=\frac{\sigma_{2}^{2}-\sigma_{3}^{2}}{\sigma_{1}^{2}-\sigma_{3}^{2}}<1$ is the mass of the pseudo-Nambu-Goldstone boson, the quantum of the $\chi_{2}$-field. The mass of the other pseudo-Nambu-Goldstone boson is 1 .

Interactions, however, come from the geometry:

$$
\begin{aligned}
& \frac{\left(\chi_{1} \partial_{\mu} \chi_{1}+\chi_{2} \partial_{\mu} \chi_{2}\right)\left(\chi_{1} \partial^{\mu} \chi_{1}+\chi_{2} \partial^{\mu} \chi_{2}\right)}{R^{2}-\chi_{1}^{2}-\chi_{2}^{2}} \simeq \\
\simeq & \frac{1}{R^{2}}\left(1+\frac{1}{R^{2}}\left(\chi_{1}^{2}+\chi_{2}^{2}\right)+\frac{1}{R^{4}}\left(\chi_{1}^{2}+\chi_{2}\right)^{2}+\cdots\right) \cdot\left(\chi_{1} \partial_{\mu} \chi_{1}+\chi_{2} \partial_{\mu} \chi_{2}\right)\left(\chi_{1} \partial^{\mu} \chi_{1}+\chi_{2} \partial^{\mu} \chi_{2}\right)
\end{aligned}
$$

which shows that $\frac{1}{R^{2}}$ is a non-dimensional coupling constant.

\subsubsection{TK1 topological $\mathbb{S}^{2}$-kinks}

Using spherical coordinates in the target space

$\chi_{1}(t, x)=R \sin \theta(t, x) \cos \varphi(t, x) \quad, \quad \chi_{2}(t, x)=R \sin \theta(t, x) \sin \varphi(t, x) \quad, \quad \chi_{3}(t, x)=R \cos \theta(t, x)$, the field equations become:

$\square \theta-\frac{1}{2} \sin 2 \theta\left(\partial^{\mu} \varphi \partial_{\mu} \varphi-\cos ^{2} \varphi-\sigma^{2} \sin ^{2} \varphi\right)=0 \quad, \quad \partial^{\mu}\left(\sin ^{2} \theta \partial_{\mu} \varphi\right)-\frac{1}{2} \bar{\sigma}^{2} \sin ^{2} \theta \sin 2 \varphi=0 \quad$. 
On the half-meridians $\varphi=\frac{\pi}{2}, \varphi=\frac{3 \pi}{2}$ and $\varphi=0, \varphi=\pi$ the field equations reduce to the sineGordon equation. Therefore, there are two types of two-component topological kinks joining the North and South poles:

1. $K 1$ kinks

$$
\begin{aligned}
& \varphi_{K_{1}}(x)=\frac{\pi}{2}, \varphi_{K_{1}^{*}}(x)=\frac{3 \pi}{2} \quad ; \quad \theta_{K_{1}}(x)=2 \arctan e^{ \pm \sigma\left(x-x_{0}\right)} \\
& \chi_{1}^{K_{1}}(x)=0 \quad, \quad \chi_{2}^{K_{1}}(x)= \pm \frac{R}{\cosh \left[\sigma\left(x-x_{0}\right)\right]} \quad, \quad \chi_{3}^{K_{1}}(x)= \pm R \tanh \left[\sigma\left(x-x_{0}\right)\right]
\end{aligned}
$$

2. $K 2$ kinks

$$
\begin{aligned}
& \varphi_{K_{2}}(x)=0, \varphi_{K_{1}^{*}}(x)=\pi \quad ; \quad \theta_{K_{2}}(x)=2 \arctan e^{ \pm\left(x-x_{0}\right)} \\
& \chi_{1}^{K_{2}}(x)= \pm \frac{R}{\cosh \left[\left(x-x_{0}\right)\right]} \quad, \quad \chi_{2}^{K_{2}}(x)=0 \quad, \quad \chi_{3}^{K_{2}}(x)= \pm R \tanh \left[\left(x-x_{0}\right)\right]
\end{aligned}
$$
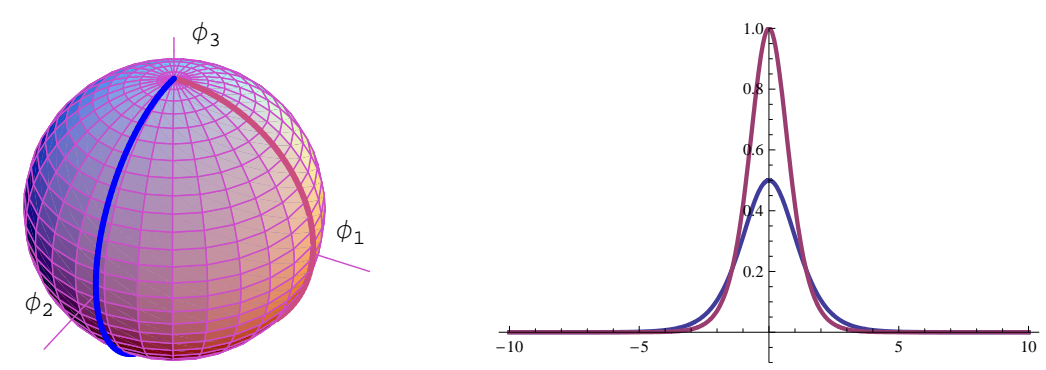

Figure 10: a) $K_{1}$ and $K_{2}\left(\sigma^{2}=\frac{1}{2}\right)$ kink orbits. b) $K_{1}$ (blue) and $K_{2}$ (red) kink energy densities

In Figure 10 the topological kink orbits are plotted, together with the corresponding Kink energy densities. The classical kink energies are:

$$
E_{K_{1}}^{C}=m \sqrt{2} R^{2} \sigma \quad, \quad E_{K_{2}}^{C}=m \sqrt{2} R^{2}
$$

and the $K_{2}$ kinks are heavier than the $K_{1}$-kinks.

\subsubsection{Kink fluctuations and one-loop mass shifts}

The geodesic deviation from the $K_{1}$ kink orbit plus the Hessian of the potential for the $K_{1}$ kink (in a parallel frame to the kink orbit) reads, see [34]:

$$
K=\left(\begin{array}{cc}
-\frac{d^{2}}{d x^{2}}+\sigma^{2}-\frac{2 \sigma^{2}}{\cosh ^{2} \sigma x} & 0 \\
0 & -\frac{d^{2}}{d x^{2}}+1-\frac{2 \sigma^{2}}{\cosh ^{2} \sigma x}
\end{array}\right)
$$

As might be expected, for parallel fluctuations to the orbit we find the same Schrödinger operator as for the sine-Gordon kink. What comes as a surprise is that the orthogonal fluctuations are also governed by an operator, which is of the transparent Pösch-Teller type of the $N=1$ class with slightly different threshold. 
The one-loop $K_{1}$ mass shift is immediately obtained from the Cahill-Comtet-Glauber formula:

$$
\begin{aligned}
E_{K_{1}}^{O L}(\sigma) & =E_{K_{1}}(\sigma)+\Delta M_{K_{1}}(\sigma)+\mathscr{O}\left(\frac{\hbar^{2}}{R^{2}}\right) \\
& =\sqrt{2} m R^{2} \sigma-\frac{\hbar m \sigma}{\sqrt{2} \pi}\left[\sin v_{1}+\frac{1}{\sigma} \sin v_{2}-v_{1} \cos v_{1}-\frac{1}{\sigma} v_{2} \cos v_{2}\right]+\mathscr{O}\left(\frac{\hbar^{2}}{R^{2}}\right)
\end{aligned}
$$

Here, $v_{1}=\arccos \left(\frac{0}{\sigma}\right)=\frac{\pi}{2}$ and $v_{2}=\arccos \left(\frac{\bar{\sigma}}{1}\right)$. The arguments are the square root of the value of the bound state eigenvalue $\bar{\sigma}^{2}=1-\sigma^{2}$ divided by the threshold of the continuous spectrum. For instance, as a function of $\sigma$ the correction reads:

$$
E_{K_{1}}^{O L}(\sigma)=\sqrt{2} m R^{2} \sigma-\frac{\hbar m \sigma}{\sqrt{2} \pi}\left[2-\frac{\bar{\sigma}}{\sigma} \arccos (\bar{\sigma})\right]+\mathscr{O}\left(\frac{\hbar^{2}}{R^{2}}\right)
$$

whereas for $\sigma=\frac{1}{2}$ we obtain :

$$
E_{K_{1}}^{O L}\left(\frac{1}{2}\right)=\frac{m}{\sqrt{2}} R^{2}-\frac{3 \hbar m}{2 \sqrt{2} \pi}\left(\frac{2}{3}-\frac{\pi}{6 \sqrt{3}}\right)+\mathscr{O}\left(\frac{\hbar^{2}}{R^{2}}\right)
$$

The second-order fluctuation operator for the $K_{2}$ kink (in a parallel frame to the kink orbit) is:

$$
K=\left(\begin{array}{cc}
-\frac{d^{2}}{d x^{2}}+1-\frac{2}{\cosh ^{2} x} & 0 \\
0 & -\frac{d^{2}}{d x^{2}}+\sigma^{2}-\frac{2}{\cosh ^{2} x}
\end{array}\right)
$$

The bound state eigenvalue $-\bar{\sigma}^{2}$ in the orthogonal direction to the orbit is negative, telling us that the $K_{2}$ kinks are unstable. The CCG formula applied to calculate the one-loop $K_{2}$ mass shift captures this fact, because $v_{2}$ ceases to be an angle and becomes arccosh $\bar{\sigma}$ :

$$
\begin{aligned}
E_{K_{2}}^{O L}(\sigma) & =E_{K_{2}}(\sigma)+\Delta M_{K_{2}}(\sigma)+\mathscr{O}\left(\frac{\hbar^{2}}{R^{2}}\right) \quad ; \quad v_{1}=\arccos (0)=\frac{\pi}{2}, v_{2}=\arccos (i \bar{\sigma}) \\
& =\sqrt{2} m R^{2}-\frac{\hbar m \sigma}{\sqrt{2} \pi}\left[\sin v_{1}+\frac{1}{\sigma} \sin v_{2}-v_{1} \cos v_{1}-\frac{1}{\sigma} v_{2} \cos v_{2}\right]+\mathscr{O}\left(\frac{\hbar}{R^{2}}\right)
\end{aligned}
$$

Therefore,

$$
E_{K_{2}}^{O L}(\sigma)=\sqrt{2} m R^{2}-\frac{\hbar m \sigma}{\sqrt{2} \pi}\left[\frac{1}{\sigma}+\sqrt{2-\sigma^{2}}-i \frac{\pi}{2} \bar{\sigma}+\bar{\sigma} \log \left(\sqrt{2-\sigma^{2}}-\bar{\sigma}\right)\right]+\mathscr{O}\left(\frac{\hbar^{2}}{R^{2}}\right)
$$

has an imaginary part, as corresponds to the energy of a resonant state in quantum physics. We end this first part by computing:

$$
E_{K_{2}}^{O L}\left(\frac{1}{2}\right)=\frac{m}{\sqrt{2}} R^{2}-\frac{\hbar m}{4 \sqrt{2} \pi}\left(4+\sqrt{7}+\sqrt{3} \log \left(\frac{\sqrt{7}-\sqrt{3}}{2}\right)-i \frac{\pi \sqrt{3}}{2}\right)+\mathscr{O}\left(\frac{\hbar^{2}}{R^{2}}\right)
$$




\section{Abelian Higgs models in a plane}

The second part will be devoted to discussing the quantum fluctuations of the topological solitons that arise in gauge theoretical models in $(2+1)$-dimensions with spontaneous breaking of the gauge symmetry. The prototype of these solitons is the Abrikosov-Nielsen-Olesen vortex, see [22], [22] . Because the spatial components of the gauge field form a purely vorticial vector field, the planar soliton is made from lumps of magnetic field. By extending these objects to the third spatial dimension magnetic flux lines arise like those existing in Type II superconductors, predicted by the phenomenological Ginzburg-Landau theory. In the relativistic Abelian Higgs model the NielsenOlesen vortices form strings that might confine magnetic monopoles. The one-loop mass shift due to quantum fluctuations of ANO vortices has been computed at the critical point between Type I and Type II superconductors in [[28], [29], and [30] using heat kernernel/zeta function regularization methods.

It was discovered in [24] that a generalization of the Higgs model, the so called semi-local Abelian Higgs model containing a doublet of complex scalar fields, also admits planar topological solitons similar to the ANO vortices. The moduli space of these solitons is, however, richer, encompassing both $\mathbb{C} P^{1}$-lumps and ANO vortices as limiting cases with very complicated mixtures of these two classes in between, see [46]. The one-loop fluctuations of self-dual semi-local topological solitons have been analyzed in [B] ] and [B2], with some surprising results. Our aim in this part is the description of these results. We remark that the semi-local Abelian Higgs model is the bosonic sector of the electro-weak theory when the Weinberg angle is $\frac{\pi}{2}$ and the $Z, W^{ \pm}$gauge fields decouple.

\subsection{The planar semi-local Abelian Higgs model}

\subsubsection{Action and field equations}

In the semi-local Abelian Higgs model there is a Higgs doublet

$$
\Phi\left(x^{\mu}\right)=\left(\begin{array}{l}
\Phi_{1}\left(x^{\mu}\right) \\
\Phi_{2}\left(x^{\mu}\right)
\end{array}\right)=\left(\begin{array}{l}
\phi_{1}\left(x^{\mu}\right)+i \phi_{2}\left(x^{\mu}\right) \\
\phi_{3}\left(x^{\mu}\right)+i \phi_{4}\left(x^{\mu}\right)
\end{array}\right): \mathbb{R}^{1,2} \longrightarrow \mathbb{C}^{2}
$$

and an Abelian gauge field:

$$
A_{\mu}\left(x^{\mu}\right) \frac{\partial}{\partial x^{\mu}}: \quad T \mathbb{R}^{1,2} \longrightarrow \quad \operatorname{Lie} \mathbb{U}(1)
$$

The action is built from the electromagnetic tensor, and interactions between the gauge and scalar fields determined from a minimal coupling principle by means of the covariant derivative and selfinteractions of the scalar field are induced by a quartic potential energy density:

$$
F_{\mu \nu}=\partial_{\mu} A_{v}-\partial_{v} A_{\mu} \quad, \quad D_{\mu} \Phi=\partial_{\mu} \Phi-i A_{\mu} \Phi \quad, \quad U(\Phi)=\frac{\lambda}{8 e^{2}}\left(\Phi^{\dagger} \Phi-1\right)^{2}
$$

The action governing the dynamics of the system is:

$$
S=\frac{v}{e} \int d x^{3}\left\{-\frac{1}{4} F_{\mu \nu} F^{\mu \nu}+\frac{1}{2}\left(D_{\mu} \Phi\right)^{\dagger} D^{\mu} \Phi-\frac{\kappa}{8}\left(\Phi^{\dagger} \Phi-1\right)^{2}\right\} \quad .
$$


There are the following (dimensional) parameters: $\left[v^{2}\right]=M$, the vacuum expectation value of the Higgs field, the scalar-vector and scalar-scalar couplings, $\left[e^{2}\right]=[\lambda]=M^{-1} L^{-2}$, and the Higgs and vector particle masses: $m^{2}=e^{2} v^{2}, \mu^{2}=\lambda v^{2}$. The ratio between masses $\kappa^{2}=\frac{\lambda}{e^{2}}$ is a very important parameter that determines whether we are in a Type I or Type II superconductivity-like regime. Needless to say, we continue working with non-dimensional fields, $\Phi, A_{\mu}$, and Minkowski coordinates $x_{\mu}, \mu=0,1,2$. The metric and volume integration are chosen such that:

$$
\begin{aligned}
& g_{\mu \nu}=\operatorname{diag}(1,-1,-1) \quad, \quad d x^{3}=d x_{0} d x_{1} d x_{2} \\
& x_{\mu} x^{\mu}=g_{\mu \nu} x^{\mu} x^{\nu}=x_{0}^{2}-x_{1}^{2}-x_{2}^{2} \quad, \quad \partial_{\mu} \partial^{\mu}=g_{\mu \nu} \partial^{\mu} \partial^{v}=\frac{\partial^{2}}{\partial x_{0}^{2}}-\frac{\partial^{2}}{\partial x_{1}^{2}}-\frac{\partial^{2}}{\partial x_{2}^{2}} \quad \text {. }
\end{aligned}
$$

The field equations are:

$$
\partial_{\mu} F^{\mu v}=i\left[\left(D^{v} \Phi\right)^{\dagger} \Phi-\Phi^{\dagger} D^{v} \Phi\right] \quad, \quad D_{\mu} D^{\mu} \Phi=\frac{\kappa^{2}}{4} \Phi\left(1-\Phi^{\dagger} \Phi\right)
$$

\subsubsection{Global and local symmetries: vacuum orbit structure}

The $\mathbb{S U}(2)$ global weak iso-spin transformations

$$
\Phi\left(x^{\mu}\right) \longrightarrow \Phi^{\prime}\left(x^{\mu}\right)=\exp \left(-\frac{i}{2} \vec{\theta} \cdot \vec{\sigma}\right) \Phi\left(x^{\mu}\right)
$$

as well as the $\mathbb{U}(1)$-gauge local transformations

$$
\Phi\left(x^{\mu}\right) \longrightarrow \Phi^{\prime}\left(x^{\mu}\right)=e^{i \alpha\left(x^{\mu}\right)} \Phi\left(x^{\mu}\right) \quad, \quad A_{\mu}\left(x^{\mu}\right) \longrightarrow A_{\mu}^{\prime}\left(x^{\mu}\right)=A_{\mu}\left(x^{\mu}\right)+\frac{\partial \alpha}{\partial x^{\mu}}\left(x^{\mu}\right)
$$

are symmetries of this system suggesting the slightly deceptive name for this model. The vacuum orbit has a very subtle structure due to the combined action of these two symmetries.

The manifold of zero energy configurations, $\Phi^{V}\left(x^{\mu}\right)=\Phi^{V}, A_{\mu}^{V}\left(x^{\mu}\right)=0_{\mu}$, is:

$$
\left(\Phi^{V}\right)^{\dagger} \Phi^{V}=\left(\Phi_{1}^{V}\right)^{*} \Phi_{1}^{V}+\left(\Phi_{2}^{V}\right)^{*} \Phi_{2}^{V}=1=\left(\phi_{1}^{V}\right)^{2}+\left(\phi_{2}^{V}\right)^{2}+\left(\phi_{3}^{V}\right)^{2}+\left(\phi_{4}^{V}\right)^{2}
$$

The Higgs vacuum orbit is the $\mathbb{S}^{3}$ unit sphere in $\mathbb{C}^{2}$ : the orbit of the point $\Phi_{0}^{V}=\left(\begin{array}{l}1 \\ 0\end{array}\right)$, the north pole of the $\mathbb{S}^{3}$ sphere, under the global $\mathbb{S U}(2)$ action, i.e., the Hopf fibre bundle:

$$
\mathbb{S}^{1} \longrightarrow \mathbb{S}^{3} \longrightarrow \mathbb{S}^{2}
$$

given by the action of a $\mathbb{U}(1)$ subgroup on each point of the $\mathbb{S}^{2}$ sphere.

Use of the Hopf coordinates $\theta_{1}, \theta_{2} \in[0,2 \pi], \psi \in\left[0, \frac{\pi}{2}\right]$ in the $\mathbb{S}^{3}$ sphere allows us to write the $\mathbb{S U}(2)$ action in the form:

$$
G \Phi_{0}^{V}=\left(\begin{array}{cc}
e^{i \theta_{1}} \sin \psi & e^{i \theta_{2}} \cos \psi \\
-e^{-i \theta_{2}} \cos \psi & e^{-i \theta_{1}} \sin \psi
\end{array}\right)\left(\begin{array}{l}
1 \\
0
\end{array}\right)=\left(\begin{array}{c}
e^{i \theta_{1}} \sin \psi \\
-e^{-i \theta_{2}} \cos \psi
\end{array}\right)
$$

such that the Higgs vacuum orbit is parametrized as follows:

$$
\phi_{1}^{V}=\cos \theta_{1} \sin \psi \quad, \quad \phi_{2}^{V}=\sin \theta_{1} \sin \psi \quad, \quad \phi_{3}^{V}=-\cos \theta_{2} \cos \psi \quad, \quad \phi_{4}^{V}=\sin \theta_{2} \cos \psi \quad .
$$


Let us consider the one form $\omega=\frac{1}{\pi}\left(\phi_{1}^{V} d \phi_{2}^{V}+\phi_{3}^{V} d \phi_{4}^{V}\right) \in \Omega^{1}\left(\mathbb{S}^{3}\right)$. The Hopf index, labeling the homotopy class of the third homotopy group of the 2 -sphere $\Pi_{3}\left(\mathbb{S}^{2}\right)=\mathbb{Z}$, is:

$$
h=\frac{1}{\pi^{2}} \int_{S^{3}} \omega \wedge d \omega=\frac{2}{\pi^{2}} \int_{S^{3}} \phi_{1}^{V} d \phi_{2}^{V} \wedge d \phi_{3}^{V} \wedge d \phi_{4}^{V}
$$

Thus,

$$
\phi_{1}^{V} d \phi_{2}^{V} \wedge d \phi_{3}^{V} \wedge d \phi_{4}^{V}=-\cos ^{2} \theta_{1} \sin ^{3} \psi \cos \psi d \psi \wedge d \theta_{1} \wedge d \theta_{2}
$$

and the Hopf index of the vacuum orbit is:

$$
h=\frac{2}{\pi^{2}}\left[2 \pi \cdot \int_{0}^{2 \pi} d \theta_{1} \cos ^{2} \theta_{1} \cdot \int_{0}^{\frac{\pi}{2}} d \psi \sin ^{3} \psi \cos \psi\right]=\frac{2}{\pi^{2}}\left[2 \pi \cdot \pi \cdot \frac{1}{4}\right]=1
$$

\subsubsection{Higgs mechanism and Feynman rules}

The choice of a point in the vacuum orbit $\mathbb{S}^{3}$ spontaneously breaks the $\mathbb{S U}(2)$-global symmetry and one would expect three Goldstone bosons. One of the three Goldstone bosons, however, will undergo the Higgs mechanism. We shift the scalar field away from the vacuum in $H\left(x^{\mu}\right)$-real Higgs-, $G\left(x^{\mu}\right)$-real Higgs ghost-, and $\varphi\left(x^{\mu}\right)$-complex Goldstone- fields:

$$
\Phi\left(x^{\mu}\right)=\left(\begin{array}{c}
1+H\left(x^{\mu}\right)+i G\left(x^{\mu}\right) \\
\sqrt{2} \varphi\left(x^{\mu}\right)
\end{array}\right)
$$

The choice of the Feynman-'t Hooft R-gauge

$$
R\left(A_{\mu}, G\right)=\partial_{\mu} A^{\mu}\left(x^{\mu}\right)+G\left(x^{\mu}\right) \quad, \quad S_{\text {g.f. }}=-\frac{1}{2} \int d^{3} x\left(\partial_{\mu} A^{\mu}\left(x^{\mu}\right)+G\left(x^{\mu}\right)\right)^{2}
$$

needs a Faddeev-Popov determinant to restore unitarity which amounts to introducing a complex ghost field:

$$
\begin{aligned}
& R\left(A_{\mu}^{\prime}, G^{\prime}\right) \simeq R\left(A_{\mu}, G\right)+\left(\square-1-H\left(x^{\mu}\right)\right) \cdot \delta \alpha\left(x^{\mu}\right) \\
& \operatorname{Det} \frac{\delta R}{\delta \alpha}=\int\left[d \chi^{*}\left(x^{\mu}\right)\right]\left[d \chi\left(x^{\mu}\right)\right] \exp \left\{i \int d x^{3} \chi^{*}\left(x^{\mu}\right)\left(\square-1-H\left(x^{\mu}\right)\right) \chi\left(x^{\mu}\right)\right\}
\end{aligned}
$$

The action becomes:

$$
\begin{aligned}
S & =\frac{v}{e} \int d^{3} x\left[-\frac{1}{2} A_{\mu}\left[-g^{\mu v}(\square+1)\right] A_{v}+\partial_{\mu} \chi^{*} \partial^{\mu} \chi-\chi^{*} \chi\right. \\
& +\frac{1}{2} \partial_{\mu} G \partial^{\mu} G-\frac{1}{2} G^{2}+\frac{1}{2} \partial_{\mu} H \partial^{\mu} H-\frac{\kappa^{2}}{2} H^{2}+\partial_{\mu} \varphi^{*} \partial^{\mu} \varphi \\
& -\frac{\kappa^{2}}{2} H\left(H^{2}+G^{2}\right)+A_{\mu}\left(\partial^{\mu} H G-\partial^{\mu} G H\right)+H\left(A_{\mu} A^{\mu}-\chi^{*} \chi\right)+i A_{\mu}\left(\varphi^{*} \partial^{\mu} \varphi-\varphi \partial^{\mu} \varphi^{*}\right) \\
& \left.+A_{\mu} A^{\mu}|\varphi|^{2}-\frac{\kappa^{2}}{8}\left(H^{2}+G^{2}\right)^{2}+\frac{1}{2}\left(G^{2}+H^{2}\right) A_{\mu} A^{\mu}-\frac{\kappa^{2}}{2}|\varphi|^{2}\left(|\varphi|^{2}+H^{2}+G^{2}+2 H\right)\right]
\end{aligned}
$$

The Feynman rules are summarized in the next two Tables. Table 4 gives the propagators in the $R$-gauge. There is a Higgs field propagating with mass $\kappa^{2}$, a Higgs ghost of mass 1 , and a vector particle with mass also 1. A Faddeev-Popov (anti-commuting) ghost of mass 1 must be included to 


\begin{tabular}{|c|c|c|c|}
\hline Particle & Field & Propagator & Diagram \\
\hline Higgs & $H(x)$ & $\frac{i e \hbar}{v\left(k^{2}-\kappa^{2}+i \varepsilon\right)}$ & - $\quad k$ \\
\hline Higgs Ghost & $G(x)$ & $\frac{i e \hbar}{v\left(k^{2}-1+i \varepsilon\right)}$ & $k$ \\
\hline Complex Goldstone & $\varphi(x)$ & $\frac{i e \hbar}{v\left(k^{2}+i \varepsilon\right)}$ & • \\
\hline Ghost & $\chi(x)$ & $\frac{i e \hbar}{v\left(k^{2}-1+i \varepsilon\right)}$ & $\bullet-\stackrel{k}{\rightarrow}-\bullet$ \\
\hline Vector Boson & $A_{\mu}(x)$ & $\frac{-i e \hbar g^{\mu v}}{v\left(k^{2}-1+i \varepsilon\right)}$ & $\sim_{e_{\mu}} \sim \sim^{k} \sim_{e_{\nu}}$ \\
\hline
\end{tabular}

Table 4: Propagators

\begin{tabular}{|c|c|c|c|c|c|c|c|}
\hline Vertex & Weight & Vertex & Weight & Vertex & Weight & Vertex & Weight \\
\hline & $-3 i \kappa^{2} \frac{1}{\hbar}$ & & $-3 i \kappa^{2} \frac{v}{\hbar \epsilon}$ & & $i\left(k^{\mu}+\right.$ & & $2 i g^{\mu v} \frac{v}{\hbar e}$ \\
\hline & $-i \kappa^{2} \frac{v}{\hbar e}$ & & $-3 i \kappa^{2} \frac{v}{\hbar c}$ & & $\left(k^{\mu}-q\right.$ & & $2 i \frac{v}{\hbar e} g^{\mu v}$ \\
\hline & $2 i \frac{v}{\hbar e} g^{\mu v}$ & & $-i \kappa^{2} \frac{v}{\hbar e}$ & & $-2 i \kappa^{2}$ & & $-i \kappa^{2} \frac{v}{\hbar e}$ \\
\hline & $-i \frac{v}{\hbar e}$ & & $2 i \frac{v}{\hbar e} g^{\mu v}$ & & $-i \kappa^{2} \frac{v}{\hbar e}$ & & $-i \kappa^{2} \frac{v}{\hbar e}$ \\
\hline
\end{tabular}

Table 5: Third- and fourth-valent vertices

compensate the non-physical Higgs ghost. Finally, there is a complex Goldstone boson, as expected from this partial Higgs mechanism. The vertices are read from the cubic and quartic terms in action (B.D) and shown in Table 5. There are accordingly three-valent and four-valent vertices. The cubic terms with derivatives in (B.]) provide weights proportional not only to the coupling constants but also dependent on the momenta. 


\subsection{Topological solitons}

The search for time-independent finite energy solutions requires the use of the Weyl gauge: $A_{0}(x)=0$; otherwise, time-dependent gauge transformations would spoil the time independence. We thus look for solutions of

$$
\text { 1) } \left.-\partial_{0}^{2} A_{i}+\partial_{i} F_{i j}=\frac{i}{2}\left(\Phi^{\dagger} D_{i} \Phi-\left(D_{i} \Phi\right)^{\dagger} \Phi\right) \quad, \quad 2\right)-\frac{1}{2} \partial_{0}^{2} \Phi+\frac{1}{2} D_{i} D_{i} \Phi=\frac{\kappa}{4} \Phi\left(\Phi^{\dagger} \Phi-1\right)
$$

such that their energy

$$
E\left(\Phi, A_{i}\right)=\int d^{2} x\left\{\frac{1}{4} F_{i j} F_{i j}+\frac{1}{2}\left(D_{i} \Phi\right)^{\dagger} D_{i} \Phi+\frac{\kappa}{8}\left(\Phi^{\dagger} \Phi-1\right)^{2}\right\}
$$

is finite.

\subsubsection{Topology of the configuration space}

The configuration space

$$
\mathscr{C}=\left\{\Phi(\vec{x}) \in \operatorname{Maps}\left(\mathbb{R}^{2}, \mathbb{C}^{2}\right), A_{i}(\vec{x}) \in \operatorname{Maps}\left(\mathbb{R}^{2}, T \mathbb{R}^{2}\right) / E\left(\Phi, A_{i}\right)<+\infty\right\}
$$

is the set of all the field configurations of finite energy. Consider polar coordinates in the plane: $r=+\sqrt{x_{1}^{2}+x_{2}^{2}}, \theta=\arctan \frac{x_{2}}{x_{1}}$. The equations

$$
\text { 1) }\left.\Phi^{\dagger} \Phi\right|_{\mathbb{S}_{\infty}^{1}}=1 \quad \text { 2) }\left.D_{i} \Phi\right|_{\mathbb{S}_{\infty}^{1}}=\left.\left(\partial_{i} \Phi-i A_{i} \Phi\right)\right|_{\mathbb{S}_{\infty}^{1}}=0
$$

are the necessary conditions to be satisfied by finite energy static field configurations at the boundary of $\mathbb{R}^{2}$ at infinity: $\mathbb{S}_{\infty}^{1} \equiv\left\{x_{1}, x_{2} / \lim _{r \rightarrow \infty}\left(x_{1}^{2}+x_{2}^{2}=r^{2}\right)\right\} \equiv \partial \mathbb{R}^{2}$. Therefore, equation (B.4(1)) determines a map from $\mathbb{S}_{\infty}^{1}$ in $\mathbb{S}^{3}$. If $\left.\Phi_{2}\right|_{\mathbb{S}_{\infty}^{1}}=0$

$$
\left.\Phi\right|_{\mathbb{S}_{\infty}^{1}}=\left(\begin{array}{c}
\left.\Phi_{1}\right|_{\mathbb{S}_{\infty}^{1}} \\
\left.\Phi_{2}\right|_{\mathbb{S}_{\infty}^{1}}
\end{array}\right)=\left(\begin{array}{c}
\left.\phi_{1}\right|_{\mathbb{S}_{\infty}^{1}}+\left.i \phi_{2}\right|_{\mathbb{S}_{\infty}^{1}} \\
\left.\phi_{3}\right|_{\mathbb{S}_{\infty}^{1}}+\left.i \phi_{4}\right|_{\mathbb{S}_{\infty}^{1}}
\end{array}\right)=\left(\begin{array}{c}
e^{i l \theta} \\
0
\end{array}\right)=\Phi_{l}^{V} \quad, \quad l \in \mathbb{Z}
$$

are all the single-valued maps complying with (B.Z(1)). Acting with the $\mathbb{S U}(2)$ matrices parametrized by Hopf coordinates we obtain the general solution by changing the base point in $\mathbb{S}^{2}$ at which $\mathbb{S}^{1}$ is fibred:

$$
G \Phi_{l}^{V}=\left(\begin{array}{cc}
e^{i \theta_{1}} \sin \psi & e^{i \theta_{2}} \cos \psi \\
-e^{-i \theta_{2}} \cos \psi & e^{-i \theta_{1}} \sin \psi
\end{array}\right)\left(\begin{array}{c}
e^{i l \theta} \\
0
\end{array}\right)=\left(\begin{array}{c}
e^{i\left(\theta_{1}+l \theta\right)} \sin \psi \\
-e^{-i\left(\theta_{2}-l \theta\right)} \cos \psi
\end{array}\right)
$$

Equation $(\mathbf{B . 4}(2))$ is consequently solved by:

$$
\begin{aligned}
\left.A_{i}\right|_{\mathbb{S}_{\infty}^{1}} & =-\left.i \Phi^{\dagger} \partial_{i} \Phi\right|_{\mathbb{S}_{\infty}^{1}}=l \frac{\partial \theta}{\partial x_{i}} \\
& =\left.\phi_{1}(\vec{x}) \frac{\partial \phi_{2}}{\partial x_{i}}(\vec{x})\right|_{\mathbb{S}_{\infty}^{1}}-\left.\phi_{2}(\vec{x}) \frac{\partial \phi_{1}}{\partial x_{i}}(\vec{x})\right|_{\mathbb{S}_{\infty}^{1}}+\left.\phi_{3}(\vec{x}) \frac{\partial \phi_{4}}{\partial x_{i}}(\vec{x})\right|_{\mathbb{S}_{\infty}^{1}}-\left.\phi_{4}(\vec{x}) \frac{\partial \phi_{3}}{\partial x_{i}}(\vec{x})\right|_{\mathbb{S}_{\infty}^{1}}
\end{aligned}
$$

showing that the vector field $A_{i}\left(x_{1}, x_{2}\right)$ is asymptotically vorticial. The integer number $l$ has correlated mathematical and physical meanings: 
1. It is the winding number of the map provided by the Higgs field at infinity:

$$
\mathbb{S}_{\infty}^{1} \quad \longrightarrow \quad \mathbb{S}_{1}^{1} \quad, \quad \mathscr{C}=\sqcup_{l \in \mathbb{Z}} \mathscr{C}_{l} \quad,
$$

where $\mathbb{S}_{1}^{1}$ is the fiber at the north pole of $\mathbb{S}^{2}$.

2. It is the magnetic flux of the field configuration:

$$
g=\oint_{\mathbb{S}_{\infty}^{1}}\left(A_{1}(\vec{x}) d x^{1}+A_{2}(\vec{x}) d x^{2}\right)=l \oint_{\mathbb{S}_{\infty}^{1}}\left(\frac{\partial \theta}{\partial x_{1}} d x^{1}+\frac{\partial \theta}{\partial x_{2}} d x^{2}\right)=l \int_{0}^{2 \pi} d \theta=2 \pi l
$$

Because the first homotopy group of a circle is non-trivial, $\Pi_{1}\left(\mathbb{S}_{1}^{1}\right)=\mathbb{Z}$, the configuration space is the union of numerable infinite disconnected sectors distinguished by the integer $l: \mathscr{C}=\bigsqcup_{l} \mathscr{C}_{l}$.

\subsubsection{Self-dual semi-local topological solitons}

At the critical point between Type II and Type I superconductivity where the masses of Higgs and vector particles are equal, $\kappa^{2}=1$, it is possible to write the energy, up to a total derivative, as a Bogomolny splitting:

$$
E=\int \frac{d^{2} x}{2}\left(\left(D_{1} \Phi \pm i D_{2} \Phi\right)^{\dagger}\left(D_{1} \Phi \pm i D_{2} \Phi\right)+\left[F_{12} \pm \frac{1}{2}\left(\Phi^{\dagger} \Phi-1\right)\right]^{2}\right)+\frac{1}{2}|g|
$$

The solutions of the first-order system of partial differential equations (B. d)

$$
D_{1} \Phi \pm i D_{2} \Phi=0 \quad, \quad F_{12} \pm \frac{1}{2}\left(\Phi^{\dagger} \Phi-1\right)=0
$$

are absolute minima of the energy and saturate the topological bound proportional to the quantized magnetic flux in each topological sector. Because the first-order vortex equations can be obtained as a dimensional reduction of the self-duality equations of Euclidean Yang-Mills theory in four dimensions, solutions of the PDE system (B.6) -that also solve the second order PDE system (B.2)are usually called self-dual.

The structure of the moduli space of solutions of (3.6) has been completely unveiled in [46], see also [45]. The parameters underlying the $4 l$ dimensional moduli space of topological solitons are the coordinates of the $l$ zeroes of $\Phi_{1}$, the coordinates of the $l-1$ zeroes of $\Phi_{2}$, and the scale and phase of $\Phi_{2}$. Full details can also be found in [B]].

\subsubsection{Self-dual semi-local topological solitons with mixed circle-symmetry}

We shall restrict ourselves to study solutions enjoying symmetry with respect to combined circle transformations in the $\mathbb{R}^{2}$ plane and the internal space $\mathbb{C}^{2}$. This symmetry is materialized by means of the ansatz:

$$
\Phi\left(x_{1}, x_{2}\right)=\left(\begin{array}{c}
f(r) e^{i l \theta} \\
|h(r)| e^{i(\omega+m \theta)}
\end{array}\right) \quad, \quad l, m \in \mathbb{Z}^{+}, \omega \in \mathbb{R} \quad, \quad A_{i}\left(x_{1}, x_{2}\right)=-l \varepsilon_{i j} \frac{\alpha(r)}{r^{2}} x_{j} .
$$

Note that by taking some care with the behavior of $\alpha$ at the origin the vector field $A_{i}\left(x_{1}, x_{2}\right)$ is divergence-free (purely rotational) in the whole plane. The PDE system (B.6) reduces to another first-order non-linear ODE system linking $f(r), \alpha(r)$, and $h(r)$ :

$$
\frac{1}{r} \frac{d \alpha}{d r}=-\frac{1}{2 l}\left(f^{2}(r)+|h(r)|^{2}-1\right) \quad, \quad \frac{d f}{d r}=\frac{l}{r} f(r)[1-\alpha(r)] \quad, \quad \frac{d|h|}{d r}=\frac{l}{r}|h|(r)\left(\frac{m}{l}-\alpha(r)\right) .
$$


Finite energy solutions, regular at the origin where the (multi) vortex sits, require us to solve (3.7) with asymptotic and core behavior:

$$
\begin{array}{lcccc}
\lim _{r \rightarrow \infty} f(r)=1 \quad & \lim _{r \rightarrow \infty} h(r)=0 \quad & \lim _{r \rightarrow \infty} \alpha(r)=1 & \\
f(0)=0 & , & |h(0)|=\left|h_{0}\right| \delta_{m, 0} & \alpha(0)=0, & m<l
\end{array}
$$

We stress that the contribution to the vorticity of the $\Phi_{2}$ field $m$ must be always smaller than the vorticity $l$ of the $\Phi_{1}$ for the topological solution to have finite energy. For later use, we give the magnetic field $B(r)=\frac{l}{2 r} \frac{d \alpha}{d r}$ and the energy density of these circle configurations:

$$
\mathscr{E}(r)=\frac{1}{8}\left(\frac{1}{l^{2}}+1\right)\left(1-f^{2}(r)-|h(r)|^{2}\right)^{2}+\frac{l^{2} f^{2}(r)}{r^{2}}(1-\alpha(r))^{2}+\frac{l^{2}|h(r)|^{2}}{r^{2}}\left(\frac{m}{l}-\alpha(r)\right)^{2}
$$

\subsubsection{Topological solutions of one quantum of magnetic flux}

We now go on to the most elementary solutions that carry a quantum of magnetic flux, or, $l=1=m+1$. We are guided by the procedure developed in [47] to solve the non-linear ODE system (B.7) with boundary conditions (B.8)-(B.9). First, we consider small values of $r$ and in the first-order differential equations we test the power series

$$
\begin{aligned}
f(r) & \equiv f_{1} \cdot r+f_{2} \cdot r^{2}+f_{3} \cdot r^{3}+f_{4} r^{4}+\cdots \\
\alpha(r) & \equiv \alpha_{1} \cdot r+\alpha_{2} \cdot r^{2}+\alpha_{3} \cdot r^{3}+\alpha_{4} \cdot r^{4}+\cdots \\
h(r) & \equiv h_{0}+h_{1} \cdot r+h_{2} \cdot r^{2}+h_{3} \cdot r^{3}+h_{4} \cdot r^{4}+\cdots
\end{aligned}
$$

where $f_{j}$ and $\alpha_{j}, j=1,2,3, \cdots$, are real, whereas $h_{j}, j=0,1,2, \cdots$, are complex coefficients. The coupled first-order ODE's are solved at this limit by (B.DU)-(B.DI)-(B.D2) if

$$
\begin{aligned}
f(r) & \simeq f_{1} \cdot r+\frac{f_{1}}{8}\left(\left|h_{0}\right|^{2}-1\right) \cdot r^{3}+\frac{f_{1}}{128}\left[\left(\left|h_{0}\right|^{2}-1\right)\left(2\left|h_{0}\right|^{2}-1\right)+4 f_{1}^{2}\right] \cdot r^{5}+\ldots \\
\alpha(r) & \simeq \frac{1}{4}\left(1-\left|h_{0}\right|^{2}\right) \cdot r^{2}-\left[\frac{1}{32}\left|h_{0}\right|^{2}\left(\left|h_{0}\right|^{2}-1\right)+\frac{1}{8} f_{1}^{2}\right] \cdot r^{4}- \\
& -\left[\frac{1}{768}\left|h_{0}\right|^{2}\left(\left|h_{0}\right|^{2}-1\right)\left(3\left|h_{0}\right|^{2}-2\right)+\frac{1}{192} f_{1}^{2}\left(5\left|h_{0}\right|^{2}-4\right)\right] \cdot r^{6}+\ldots \\
h(r) & \simeq h_{0}+\frac{h_{0}}{8}\left(\left|h_{0}\right|^{2}-1\right) \cdot r^{2}+\frac{h_{0}}{128}\left[\left(\left|h_{0}\right|^{2}-1\right)\left(2\left|h_{0}\right|^{2}-1\right)+4 f_{1}^{2}\right] \cdot r^{4}+\ldots
\end{aligned} .
$$

We stress that $h_{0} \in[0,1]$ is determined by the behavior of the solution at the origin such that only a free parameter, $f_{1}$, is left. Second, a numerical scheme is implemented by setting a boundary condition at a non-singular point of the ODE system, which is obtained from the power series for a small value of $r$ ( $r=0.001$ in our case). This scheme prompts a shooting procedure by varying $f_{1}$, where the correct asymptotic behavior of the solutions is obtained setting a optimal value for $f_{1}$ for a given value of $h_{0}$. Finally, the first-order ODE system is solved for long $r$ by means of a power series in $\frac{1}{r}$ :

$$
f(r)=\sum_{j=0} f^{j} \cdot r^{-j} \quad \alpha(r)=\sum_{j=0} \alpha^{j} \cdot r^{-j} \quad h(r)=\sum_{j=1} h^{j} \cdot r^{-j}
$$


with the result that

$$
\begin{aligned}
f(r) \simeq & 1-\frac{\left|h^{1}\right|^{2}}{2} \cdot r^{-2}+\left(-2\left|h^{1}\right|^{2}+\frac{3}{8}\left|h^{1}\right|^{4}\right) \cdot r^{-4}+\left|h^{1}\right|^{2}\left(-32+5\left|h^{1}\right|^{2}-\frac{5}{16}\left|h^{1}\right|^{4}\right) \cdot r^{-6}+ \\
+ & \left|h^{1}\right|^{2}\left(-1152+158\left|h^{1}\right|^{2}-\frac{35}{4}\left|h^{1}\right|^{4}+\frac{35}{128}\left|h^{1}\right|^{6}\right) \cdot r^{-8}+\ldots \\
\alpha(r) \simeq & 1-\left|h^{1}\right|^{2} \cdot r^{-2}+\left|h^{1}\right|^{2}\left(-8+\left|h^{1}\right|^{2}\right) \cdot r^{-4}+\left|h^{1}\right|^{2}\left(-192+24\left|h^{1}\right|^{2}-\left|h^{1}\right|^{4}\right) \cdot r^{-6} \\
& +\left|h^{1}\right|^{2}\left(9216+1120\left|h^{1}\right|^{2}-48\left|h^{1}\right|^{4}+\left|h^{1}\right|^{6}\right) \cdot r^{-8}+\ldots \\
|h(r)| \simeq \simeq & |h|^{1} \cdot r^{-1}+-\frac{\left|h^{1}\right|^{3}}{2} \cdot r^{-3}+\left|h^{1}\right|^{3}\left(-2+\frac{3}{8}\left|h^{1}\right|^{2}\right) \cdot r^{-5}+ \\
+ & \left|h^{1}\right|^{3}\left(-32+5\left|h^{1}\right|^{2}-\frac{5}{16}\left|h^{1}\right|^{4}\right) \cdot r^{-7}+\ldots
\end{aligned}
$$

Again, only one free parameter $h^{1}$ is left. The value of $h^{1}$ is fixed by demanding the continuity of the solution at intermediate distances ( $r=15$ in our case) obtained by gluing the short- $r$ and long$r$ approximations. In particular, this has the important implication that $\left|h_{0}\right|=0 \Rightarrow\left|h^{1}\right|=0$ linking the null value of $\left|h_{0}\right|$, which gives the embedded ANO vortex, with the null value of the constant $\left|h^{1}\right|$ setting the behavior of the solution for very long $r$. Another important remark is that the long $r$ behavior of self-dual semi-local defects differs from the long $r$ behavior of self-dual ANO vortices that decay exponentially.

The following Figures show the results obtained with this procedure for several values of $h_{0}$. Note that $h_{0}=0$ for the ANO vortices, and $h_{0}=1$ for the $\mathrm{CP}^{1}$-lumps. It may be observed in the graphics that the field profiles reach their vacuum values at distances of the order of $r=$ 15. Consequently, practically identical numerical solutions would be generated by sewing the numerical to the asymptotic solution at $r$ greater than 15 .
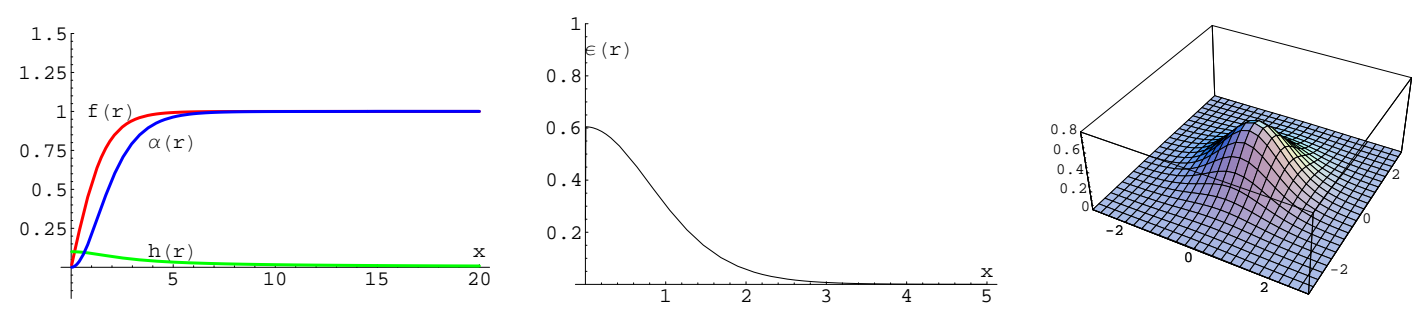

a) Functions $f(r), h(r)$ and $\alpha(r)$ and $b), c)$ Energy density for $h_{0}=0.1$
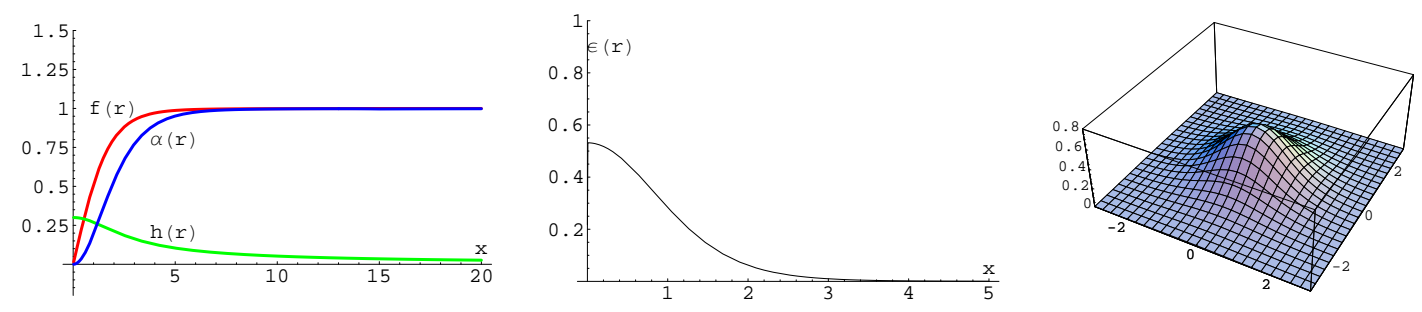

a) Functions $f(r), h(r)$ and $\alpha(r)$ and $b), c)$ Energy density for $h_{0}=0.3$ 

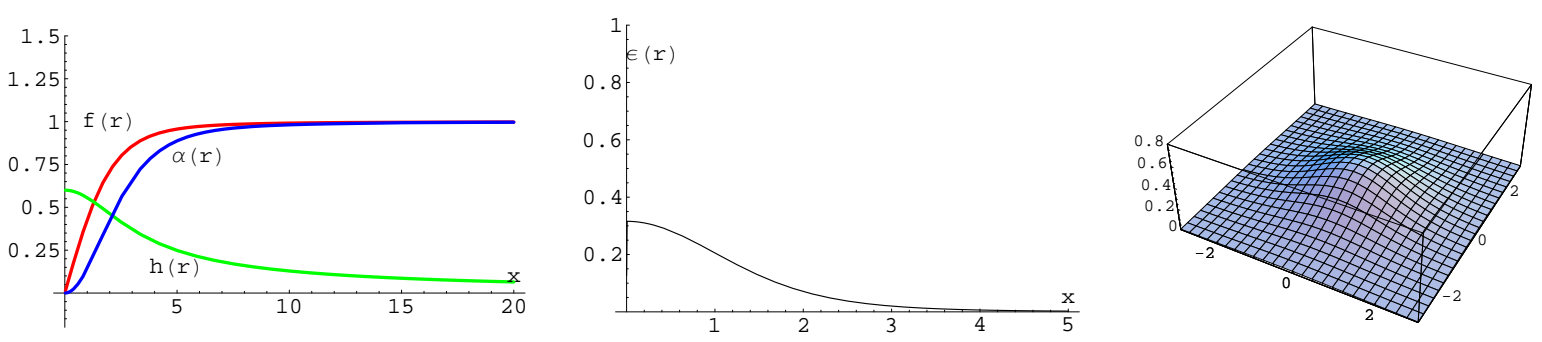

a) Functions $f(r), h(r)$ and $\alpha(r)$ and $b), c)$ Energy density for $h_{0}=0.6$
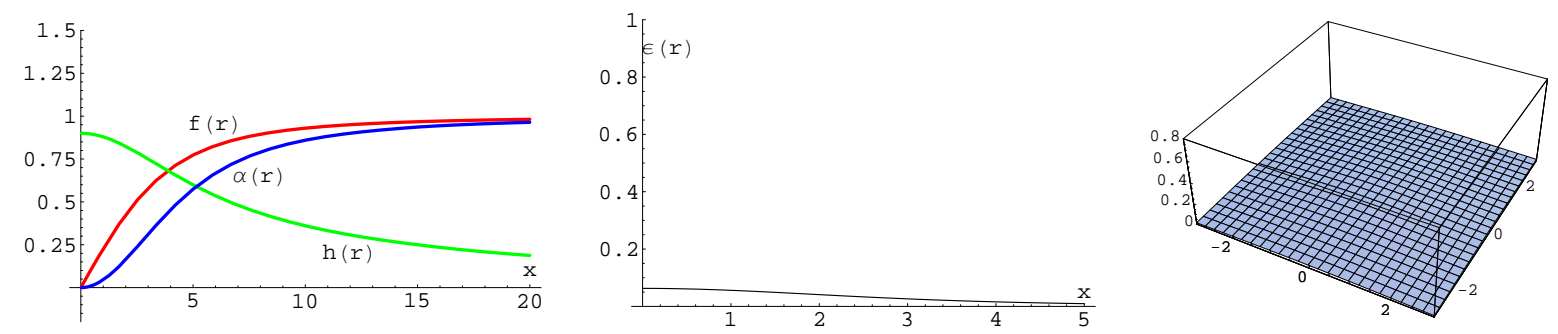

a) Functions $f(r), h(r)$ and $\alpha(r)$ and $b), c)$ Energy density for $h_{0}=0.9$

Figure 11: Field Profiles and Energy Densities for Semi-local Topological Defects

\subsubsection{Deformation of the first-order equations}

It is interesting at this point to consider small deformations of the solutions of the (13.6) system

$$
\Phi(\vec{x})=S(\vec{x})+\delta S(\vec{x}) \quad, \quad A_{j}(\vec{x})=V_{j}(\vec{x})+\delta a_{j}(\vec{x})
$$

which are still solutions of the same PDE system. The necessary and sufficient conditions for this are tantamount to the linear PDE system:

$$
\begin{aligned}
& -\partial_{2} \delta a_{1}+\partial_{1} \delta a_{2}+\frac{1}{2}\left(S^{\dagger} \delta S+\delta S^{\dagger} S\right)=0 \\
& \left(\frac{\partial}{\partial x_{1}}-i V_{1}(\vec{x})+i \frac{\partial}{\partial x_{2}}+V_{2}(\vec{x})\right) \delta S-i\left(\delta a_{1}+i \delta a_{2}\right) S=0
\end{aligned}
$$

Pure gauge fluctuations are discarded from the solutions of (B.13) by setting the background gauge:

$$
\partial_{j} \delta a_{j}(\vec{x})+\frac{i}{2}\left(S^{\dagger} \delta S-\delta S^{\dagger} S\right)=0
$$

The tangent space to the moduli space of self-dual topological solitons with a given magnetic charge $2 \pi l$ is therefore the kernel of the first-order deformation operator $\mathscr{D}$ :

$$
\mathscr{D} \xi(\vec{x})=\left(\begin{array}{cccccc}
-\partial_{2} & \partial_{1} & S_{1}^{1} & S_{1}^{2} & S_{2}^{1} & S_{2}^{2} \\
-\partial_{1}-\partial_{2} & -S_{1}^{2} & S_{1}^{1} & -S_{2}^{2} & S_{2}^{1} \\
S_{1}^{1} & -S_{1}^{2} & -\partial_{2}+V_{1} & -\partial_{1}-V_{2} & 0 & 0 \\
S_{1}^{2} & S_{1}^{1} & \partial_{1}+V_{2} & -\partial_{2}+V_{1} & 0 & 0 \\
S_{2}^{1} & -S_{2}^{2} & 0 & 0 & -\partial_{2}+V_{1} & -\partial_{1}-V_{2} \\
S_{2}^{2} & S_{2}^{1} & 0 & 0 & \partial_{1}+V_{2} & -\partial_{2}+V_{1}
\end{array}\right)\left(\begin{array}{l}
\delta a_{1}(\vec{x}) \\
\delta a_{2}(\vec{x}) \\
\delta S_{1}^{1}(\vec{x}) \\
\delta S_{1}^{2}(\vec{x}) \\
\delta S_{2}^{1}(\vec{x}) \\
\delta S_{2}^{2}(\vec{x})
\end{array}\right)=0
$$


Accordingly, $\triangle_{+}=\mathscr{D}^{\dagger} \mathscr{D}$ is the following $6 \times 6$ matrix partial differential operator:

$$
\begin{aligned}
& \triangle_{+}=\left(\begin{array}{cccccc}
A & 0 & -2 \nabla_{1} S_{1}^{2} & 2 \nabla_{1} S_{1}^{1} & -2 \nabla_{1} S_{2}^{2} & 2 \nabla_{1} S_{2}^{1} \\
0 & A & -2 \nabla_{2} S_{1}^{2} & 2 \nabla_{2} S_{1}^{1} & -2 \nabla_{2} S_{2}^{2} & 2 \nabla_{2} S_{2}^{1} \\
-2 \nabla_{1} S_{1}^{2} & -2 \nabla_{2} S_{1}^{2} & B & -2 V_{k} \partial_{k} & S_{1}^{1} S_{2}^{1}+S_{1}^{2} S_{2}^{2} & S_{1}^{1} S_{2}^{2}-S_{1}^{2} S_{2}^{1} \\
2 \nabla_{1} S_{1}^{1} & 2 \nabla_{2} S_{1}^{1} & 2 V_{k} \partial_{k} & B & -S_{1}^{1} S_{2}^{2}+S_{1}^{2} S_{2}^{1} & S_{1}^{1} S_{2}^{1}+S_{1}^{2} S_{2}^{2} \\
-2 \nabla_{1} S_{2}^{2} & -2 \nabla_{2} S_{2}^{2} & S_{1}^{1} S_{2}^{1}+S_{1}^{2} S_{2}^{2} & -S_{1}^{1} S_{2}^{2}+S_{1}^{2} S_{2}^{1} & C & -2 V_{k} \partial_{k} \\
2 \nabla_{1} S_{2}^{1} & 2 \nabla_{2} S_{2}^{1} & S_{1}^{1} S_{2}^{2}-S_{1}^{2} S_{2}^{1} & S_{1}^{1} S_{2}^{1}+S_{1}^{2} S_{2}^{2} & 2 V_{k} \partial_{k} & C
\end{array}\right) \\
& A=-\partial_{k} \partial_{k}+\left|S_{1}\right|^{2}+\left|S_{2}\right|^{2}, j, k=1,2 \quad, \quad \partial_{k} \partial_{k}=\frac{\partial^{2}}{\partial x_{1}^{2}}+\frac{\partial^{2}}{\partial x_{2}^{2}} \quad, \quad V_{k}(\vec{x}) V_{k}(\vec{x})=V_{1}^{2}(\vec{x})+V_{2}^{2}(\vec{x}) \\
& B=-\partial_{k} \partial_{k}+\frac{1}{2}\left(3\left|S_{1}\right|^{2}+\left|S_{2}\right|^{2}+2 V_{k} V_{k}-1\right) \quad, \quad C=-\partial_{k} \partial_{k}+\frac{1}{2}\left(\left|S_{1}\right|^{2}+3\left|S_{2}\right|^{2}+2 V_{k} V_{k}-1\right) \\
& \nabla_{j} S_{M}^{A}=\partial_{j} S_{M}^{A}+\varepsilon^{A B} V_{j} S_{M}^{B} \quad, \quad M=1,2, A, B=1,2 \quad, \quad \varepsilon^{12}=-\varepsilon^{21}=1, \varepsilon^{11}=\varepsilon^{22}=0 .
\end{aligned}
$$

One easily checks that $\triangle_{+}$has a supersymmetric partner ${ }^{3}: \triangle_{-}=\mathscr{D}^{\dagger}$ :

$$
\begin{gathered}
\triangle_{-}=\left(\begin{array}{cccccc}
A & 0 & 0 & 0 & 0 & 0 \\
0 & A & 0 & 0 & 0 & 0 \\
0 & 0 & B_{-} & -2 V_{k} \partial_{k} & S_{1}^{1} S_{2}^{1}+S_{1}^{2} S_{2}^{2} & S_{1}^{1} S_{2}^{2}-S_{1}^{2} S_{2}^{1} \\
0 & 0 & 2 V_{k} \partial_{k} & B_{-} & -S_{1}^{1} S_{2}^{2}+S_{1}^{2} S_{2}^{1} & S_{1}^{1} S_{2}^{1}+S_{1}^{2} S_{2}^{2} \\
0 & 0 & S_{1}^{1} S_{2}^{1}+S_{1}^{2} S_{2}^{2} & -S_{1}^{1} S_{2}^{2}+S_{1}^{2} S_{2}^{1} & C_{-} & -2 V_{k} \partial_{k} \\
0 & 0 & S_{1}^{1} S_{2}^{2}-S_{1}^{2} S_{2}^{1} & S_{1}^{1} S_{2}^{1}+S_{1}^{2} S_{2}^{2} & 2 V_{k} \partial_{k} & C_{-}
\end{array}\right) \quad, \quad V_{k} \partial_{k}=V_{1}(\vec{x}) \frac{\partial}{\partial x_{1}}+V_{1}(\vec{x}) \frac{\partial}{\partial x_{1}} \\
B_{-}=-\partial_{k} \partial_{k}+\frac{1}{2}\left(\left|S_{1}\right|^{2}-\left|S_{2}\right|^{2}+2 V_{k} V_{k}+1\right) \quad, \quad C_{-}=-\partial_{k} \partial_{k}+\frac{1}{2}\left(-\left|S_{1}\right|^{2}+\left|S_{2}\right|^{2}+2 V_{k} V_{k}+1\right) .
\end{gathered}
$$

The index of the deformation operator - ind $\mathscr{D}=\operatorname{dimKer} \mathscr{D}-\operatorname{dimKer} \mathscr{D}^{\dagger}-$ is in this case equal to the dimension of $\operatorname{Ker} \triangle_{+}$because $\operatorname{dim} \operatorname{Ker} \mathscr{D}^{\dagger}=0, \triangle_{-}$being definite positive.

Because $\triangle_{+}$and $\triangle_{-}$are iso-spectral up to zero modes the index of $\mathscr{D}$ can be regularized in the following form:

$$
\text { index } \mathscr{D}=\lim _{\beta \rightarrow 0}\left[\operatorname{Tr}_{L^{2}} e^{-\beta \triangle_{+}}-\operatorname{Tr}_{L^{2}} e^{-\beta \triangle_{-}}\right] .
$$

Let us use the heat trace expansion: $\operatorname{Tr}_{L^{2}} e^{-\beta \triangle_{ \pm}}=\operatorname{Tr}_{L^{2}} e^{-\beta \triangle} \sum_{n=0}^{\infty} c_{n}\left[\triangle_{ \pm}\right] \beta^{n}$,

$$
\triangle=\left(\begin{array}{cccccc}
-\partial_{k} \partial_{k}+1 & 0 & 0 & 0 & 0 & 0 \\
0 & -\partial_{k} \partial_{k}+1 & 0 & 0 & 0 & 0 \\
0 & 0 & -\partial_{k} \partial_{k}+1 & 0 & 0 & 0 \\
0 & 0 & 0 & -\partial_{k} \partial_{k}+1 & 0 & 0 \\
0 & 0 & 0 & 0 & -\partial_{k} \partial_{k} & 0 \\
0 & 0 & 0 & 0 & 0 & -\partial_{k} \partial_{k}
\end{array}\right)
$$

Because

$$
\operatorname{Tr}_{L^{2}} e^{-\beta \triangle}=\frac{e^{-\beta}}{\pi}+\frac{1}{2 \pi \beta} \quad, \quad c_{0}\left[\triangle_{ \pm}\right]=6 l^{2}, \quad, \quad c_{1}\left[\triangle_{ \pm}\right]=\int d x^{2} \operatorname{tr}\left(\triangle_{ \pm}(\vec{x})-\triangle\right)
$$

\footnotetext{
${ }^{3}$ This is an example of hidden bosonic supersymmetry [48]].
} 
we obtain:

$$
\operatorname{ind} \mathscr{D}=-\frac{1}{\pi} \int d x^{2} \operatorname{tr}\left(\triangle_{+}(\vec{x})-\triangle_{-}(\vec{x})\right)=\frac{2}{\pi} \int d x^{2}\left(\left|S_{1}\right|^{2}+\left|S_{2}\right|^{2}-1\right)=\frac{2}{\pi} \int d x^{2} F_{12}=4 l .
$$

In the derivation of (B.14) we have used the vortex equation (B.6) and tr means trace in the matrix sense. We find that the number of zero modes is twice the magnetic flux $(\bmod \pi)$, in perfect agreement with the number of parameters of the self-dual topological solitons.

\subsection{One-loop correction to the masses of semi-local self-dual topological solitons (SSTS)}

\subsubsection{SSTS fluctuations}

Let us now consider time-dependent small fluctuations of the self-dual topological solitons:

$$
\Phi(\vec{x})=S(\vec{x})+\delta S\left(x_{0}, \vec{x}\right) \quad, \quad A_{j}(\vec{x})=V_{j}(\vec{x})+\delta a_{j}\left(x_{0}, \vec{x}\right) \quad, \quad \chi(\vec{x})=\delta \chi(\vec{x})
$$

In order to discard pure gauge fluctuations we impose the Weyl/background gauge condition (the $\mathrm{R}$ gauge in the topological sector of magnetic flux 1):

$$
A_{0}\left(x_{0}, \vec{x}\right)=0 \quad, \quad \partial_{j} \delta a_{j}\left(x_{0}, \vec{x}\right)+\frac{i}{2}\left(S^{\dagger}(\vec{x}) \delta S\left(x_{0}, \vec{x}\right)-\delta S^{\dagger}\left(x_{0}, \vec{x}\right) S(\vec{x})\right)=0 \quad .
$$

The classical energy up to $\mathscr{O}\left(\delta^{3}\right)$ order (one-loop) of the SSTS fluctuations is:

$$
H^{(2)}=H_{B}^{(2)}+H_{F}^{(2)}=\frac{v^{2}}{2} \int d^{2} x\left\{\frac{\partial \delta \xi^{T}}{\partial x_{0}} \frac{\partial \delta \xi}{\partial x_{0}}+\delta \xi^{T} K \delta \xi+\delta \chi^{*} K^{G} \delta \chi\right\}
$$

where

$$
\delta \xi^{T}\left(x_{0}, \vec{x}\right)=\left(\delta a_{1}\left(x_{0}, \vec{x}\right) \delta a_{2}\left(x_{0}, \vec{x}\right) \quad \delta S_{1}^{1}\left(x_{0}, \vec{x}\right) \quad \delta S_{1}^{2}\left(x_{0}, \vec{x}\right) \delta S_{2}^{1}\left(x_{0}, \vec{x}\right) \delta S_{2}^{2}\left(x_{0}, \vec{x}\right)\right)
$$

is a file vector assembling the fluctuations of the two polarizations of vector particles, the Higgs and Higgs ghost fluctuations, and Goldstone fluctuations around the topological soliton solution, i.e., the bosonic fluctuations and the second-order operator determining the small fluctuations of these extended objects is precisely $K=\triangle_{+}$.

We shall impose periodic boundary conditions on the fluctuations $\delta \xi_{a}\left(x_{1}, x_{2}\right)=\delta \xi_{a}\left(x_{1}+\right.$ $\left.l, x_{2}+l\right), a=1,2, \cdots, 6, l=m L$. Therefore, $K$ acts on the Hilbert space $L^{2}=\bigoplus_{a=1}^{6} L_{a}^{2}\left(\mathbb{S}^{1} \otimes \mathbb{S}^{1}\right)$. Assuming the ortho-normality and completeness of the eigenfunctions of $K, K \xi_{n}(\vec{x})=\varepsilon_{n}^{2} \xi_{n}(\vec{x})$, in the sub-space orthogonal to its kernel, one finds the quantum Hamiltonian of the one-loop bosonic fluctuations:

$$
\hat{H}_{B}^{(2)}=\hbar m \sum_{\operatorname{Spec}_{+} K} \varepsilon_{n}\left(\hat{B}_{n}^{\dagger} \hat{B}_{n}+\frac{1}{2}\right)
$$

where $\left[\hat{B}_{n}^{\dagger}, \hat{B}_{m}\right]=\delta_{n m}$ are the expansion coefficients promoted to creation and annihilation operators. The index theorem argument in the previous Section shows that the number of normalizable zero modes is: $\operatorname{dimKer} \mathscr{D}=4 l$ where $\mathscr{D}$ is the partial differential operator arising in the deformation of the first-order equation. Because $K=\mathscr{D}^{\dagger} \mathscr{D}$, standard supersymmetry strategies allow us to conclude that the spectrum of $K$ is formed by $4 l$ zero modes and positive eigenvalues giving rise to $\mathrm{Spec}_{+} K$. 
The topological soliton ground state is a coherent state annihilated by all destruction operators:

$$
\hat{B}_{n}|0 ; T S\rangle=0, \forall n \quad \Rightarrow \quad \begin{aligned}
& \hat{\Phi}\left(x_{0}, \vec{x}\right)|0 ; T S\rangle=S(\vec{x})|0 ; T S\rangle \\
& \hat{A}_{i}\left(x_{0}, \vec{x}\right)|0 ; T S\rangle=V_{i}(\vec{x})|0 ; T S\rangle
\end{aligned}
$$

Therefore, the bosonic energy of the ground state in a topological sector of magnetic charge $l \neq 0$ is:

$$
\left\langle 0 ; T S\left|\hat{H}_{B}^{(2)}\right| T S ; 0\right\rangle=\frac{\hbar m}{2} \operatorname{Tr}_{L^{2}} K^{\frac{1}{2}} \quad .
$$

In the Figure 12 we show the diagonal potential wells/barriers in the differential operator $K$ for magnetic flux $2 \pi$. The vector bosons "essentially" feel -there are also non-diagonal terms- the potential $A$; Higgs bosons and Higgs ghosts feel the potential $B$, and the Goldstone bosons move along the potential $C$. Leaving apart the non-diagonal exchange interactions, all three types of particles move through attractive potentials, exponentially decaying to their vacuum values if the background is the ANO vortex, $h_{0}=0$. If the background is a $h_{0}=0.3$ topological soliton, both vector and Goldstone bosons move in less attractive potential wells that, moreover, only decay as some negative power of $r$ near infinity. For $h_{0}=0.9$, a value giving almost a $\mathbb{C P}^{1}$-lump, the vector bosons pass through the background feeling a extremely weak attraction, the Higgs boson and ghost note a considerably weaker attraction as compared to the attraction of the previous backgrounds, and the Goldstone bosons are repelled by the topological soliton. The decay at infinity is extremely slow. The analysis of these physical features will give qualitative support to our results on the one-loop corrections to be presented later.
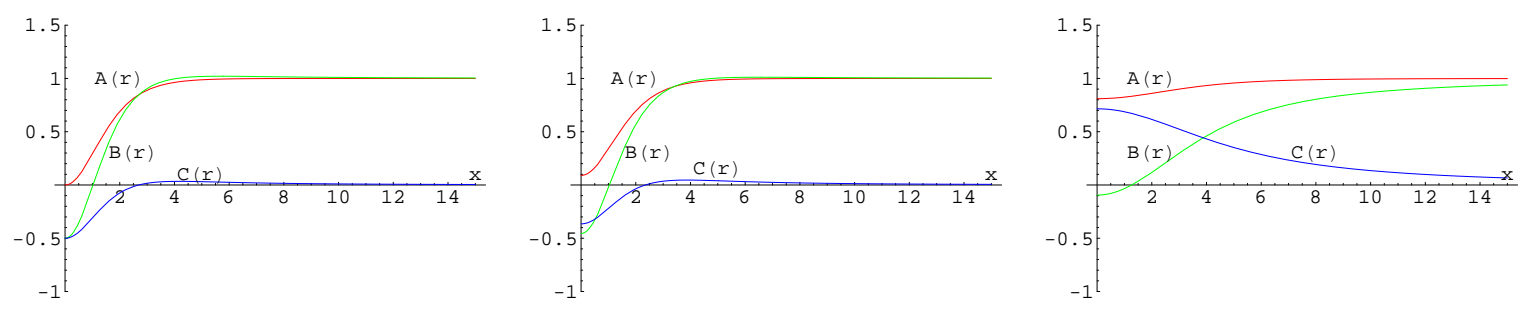

Figure 12: Potential wells for $l=1 V_{A}(r), V_{B}(r), V_{C}(r):$ a) $h_{0}=0.0$, b) $h_{0}=0.3$, c) $h_{0}=0.9$

It remains to get rid off the contribution of the Higgs ghosts by considering the ground state energy of the (fermionic) Faddeev-Popov ghosts. The FP ghost fluctuations (with PBC) are determined by the Schödinger operator:

$$
K^{G}=-\partial_{k} \partial_{k}+\left|S_{1}\right|^{2}+\left|S_{2}\right|^{2} \quad, \quad \delta \chi\left(x_{1}, x_{2}\right)=\delta \chi\left(x_{1}+l, x_{2}+l\right) \quad,
$$

such that $K^{G}$ acts on $L_{G}^{2}=L^{2}\left(\mathbb{S}^{1} \otimes \mathbb{S}^{1}\right)$. Again assuming the ortho-normality and completeness of the eigenfunctions of $K^{G}, K^{G} \chi_{n}(\vec{x})=\varepsilon_{n}^{2} \chi_{n}(\vec{x})$, in the sub-space orthogonal to its kernel, one finds the quantum Hamiltonian of the one-loop fermionic fluctuations:

$$
\hat{H}_{F}^{(2)}=\hbar m \sum_{\operatorname{Spec}_{+} K^{G}} \varepsilon_{n}\left(\hat{C}_{n}^{\dagger} \hat{C}_{n}-\frac{1}{2}\right),
$$

where $\left\{\hat{C}_{n}^{\dagger}, \hat{C}_{m}\right\}=\delta_{n m}$ are the expansion coefficients promoted to fermionic creation and annihilation operators. There are no fermionic ghosts in the topological soliton ground state. Henceforth, 
$\hat{C}_{n}|0 ; T S\rangle=0, \forall n$ and the ground state energy of the topological soliton is:

$$
\left\langle 0 ; T S\left|\hat{H}_{B}^{(2)}\right| 0 ; T S\right\rangle+\left\langle 0 ; T S\left|\hat{H}_{F}^{(2)}\right| 0 ; T S\right\rangle=\frac{\hbar m}{2}\left(\operatorname{Tr}_{L^{2}} K^{\frac{1}{2}}-\operatorname{Tr}_{L^{2}}\left(K^{G}\right)^{\frac{1}{2}}\right)=\frac{\hbar m}{2} \operatorname{STr}_{L^{2}} K^{\frac{1}{2}}
$$

\subsubsection{Vacuum fluctuations}

It is instructive to specify this analysis for the vacuum fluctuations:

$$
S^{V}(\vec{x})=\left(\begin{array}{l}
1 \\
0
\end{array}\right) \quad, \quad V_{i}^{V}(\vec{x})=0_{i} \quad .
$$

In this case the second-order operator fluctuations are $K_{0}=\triangle$ and $K_{0}^{G}=-\partial_{k} \partial_{k}+1$ such that the spectrum is completely known. In a normalization square of area $l^{2}$, periodic boundary conditions on the fluctuations plus canonical quantization produce the following bosonic and fermionic free Hamiltonians:

$$
\begin{aligned}
& \hat{H}_{B}^{(2)}=\hbar m \sum_{a=1}^{6} \sum_{n_{1} \in \mathbb{Z}} \sum_{n_{2} \in \mathbb{Z}} \omega_{a}\left(n_{1}, n_{2}\right)\left(\hat{b}_{n_{1}}^{a \dagger} \hat{b}_{n_{1}}^{a}+\hat{b}_{n_{2}}^{a \dagger} \hat{b}_{n_{2}}^{a}+1\right) \quad, \quad\left[\hat{b}_{n_{\alpha}}^{a \dagger}, \hat{b}_{m_{\beta}}^{c}\right]=\delta^{a c} \delta_{n_{\alpha} m_{\beta}} \\
& \hat{H}_{F}^{(2)}=\hbar m \sum_{n_{1} \in \mathbb{Z}} \sum_{n_{2} \in \mathbb{Z}} \omega\left(n_{1}, n_{2}\right)\left(\hat{c}_{n_{1}}^{\dagger} \hat{c}_{n_{1}}+\hat{c}_{n_{2}}^{\dagger} \hat{c}_{n_{2}}-1\right) \quad, \quad\left\{\hat{c}_{n_{\alpha}}^{\dagger}, \hat{c}_{m_{\beta}}\right\}=\delta_{n_{\alpha} m_{\beta}} \\
& \omega\left(n_{1}, n_{2}\right)=\omega_{a}\left(n_{1}, n_{2}\right)=\frac{4 \pi^{2}}{l^{2}}\left(n_{1}^{2}+n_{2}^{2}\right)+1, a=1,2,3,4 \\
& \omega_{a}\left(n_{1}^{2}, n_{2}^{2}\right)=\frac{4 \pi^{2}}{l^{2}}\left(n_{1}^{2}+n_{2}^{2}\right), a=5,6 .
\end{aligned}
$$

Therefore, the ground state in the vacuum sector is also a coherent state:

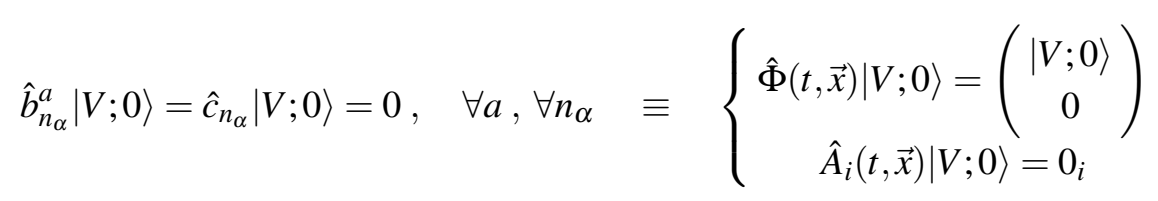

such that the ground state energy follows immediately:

$$
\left\langle 0 ; V\left|\left(\hat{H}_{B}^{(2)}+\hat{H}_{F}^{(2)}\right)\right| V ; 0\right\rangle=\operatorname{Tr}_{L^{2}} K_{0}^{\frac{1}{2}}-\operatorname{Tr}_{L^{2}}\left(K_{0}^{G}\right)^{\frac{1}{2}}=\operatorname{STr}_{L^{2}}\left(K_{0}\right)^{\frac{1}{2}} \quad .
$$

It is clear that the ghosts cancel the contribution to the vacuum energy of the non-physical Higgs ghosts and render the theory unitary.

\subsection{Zero point energy and mass renormalizations}

\subsubsection{Topological soliton Casimir energy}

Subtracting the ground state vacuum energy from the ground state energy of the topological solitons

$$
\begin{aligned}
\triangle E_{\mathrm{TS}}^{C} & =\left\langle 0 ; T S\left|\left(\hat{H}_{B}^{(2)}+\hat{H}_{F}^{(2)}\right)\right| T S ; 0 \mid\right\rangle-\left\langle 0 ; V\left|\left(\hat{H}_{B}^{(2)}+\hat{H}_{F}^{(2)}\right)\right| V ; 0 \mid\right\rangle \\
& =\triangle E_{\mathrm{TS}}-\triangle E_{0}=\frac{\hbar m}{2}\left(\mathrm{~S} \operatorname{Tr} K^{\frac{1}{2}}-\mathrm{S} \operatorname{Tr} K_{0}^{\frac{1}{2}}\right)
\end{aligned}
$$

one formally measures the semi-local self-dual topological soliton Casimir energy. 


\subsubsection{Mass renormalization counter-terms}

In $(2+1)$-dimensions, the semi-local Abelian Higgs model is super-renormalizable. This means that there are a finite number of divergent graphs. We list the one-loop graphs that diverge.

1. Higgs boson tadpole:

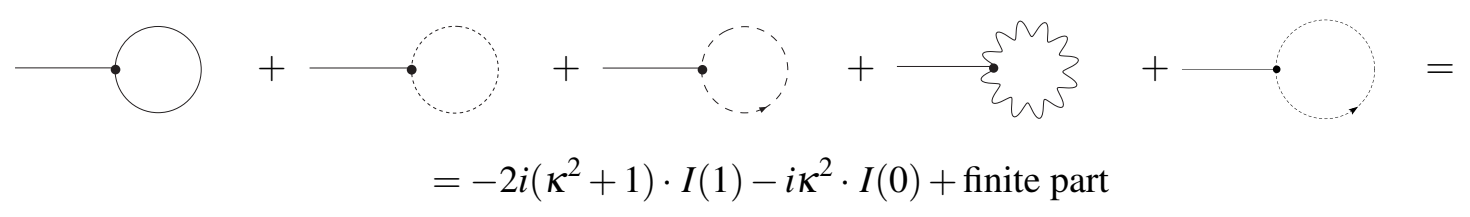

2. Higgs boson self-energy:

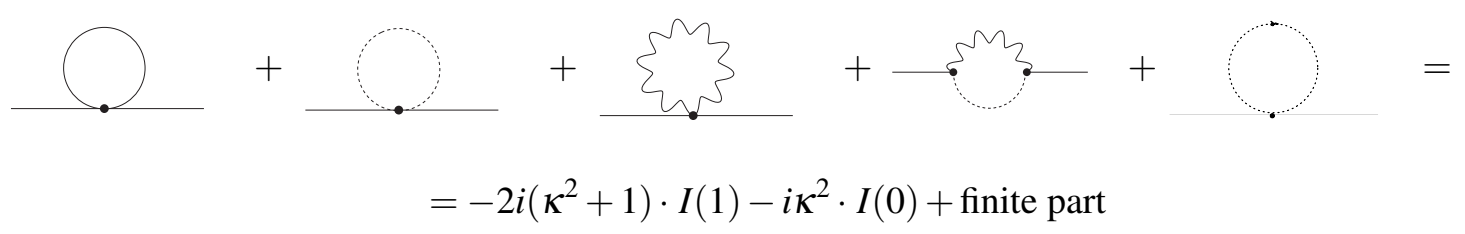

3. Higgs ghost self-energy:

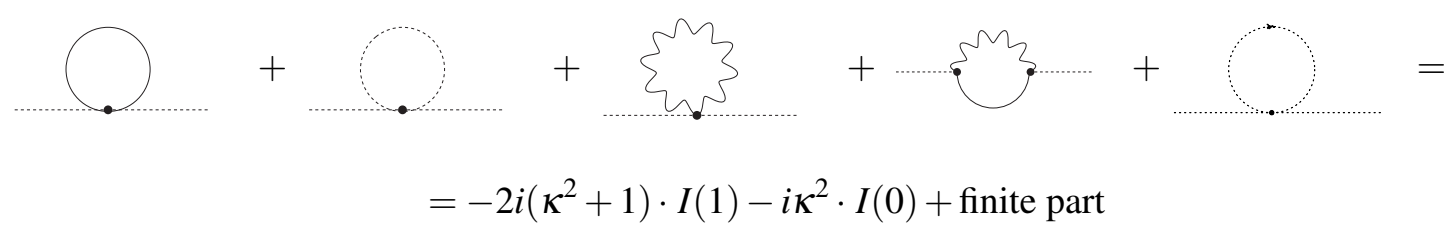

4. Goldstone boson self-energy:

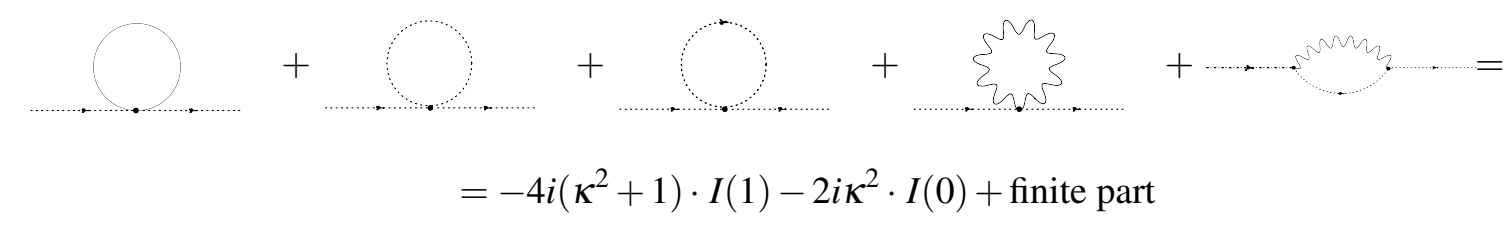

5. Vector boson self-energy:

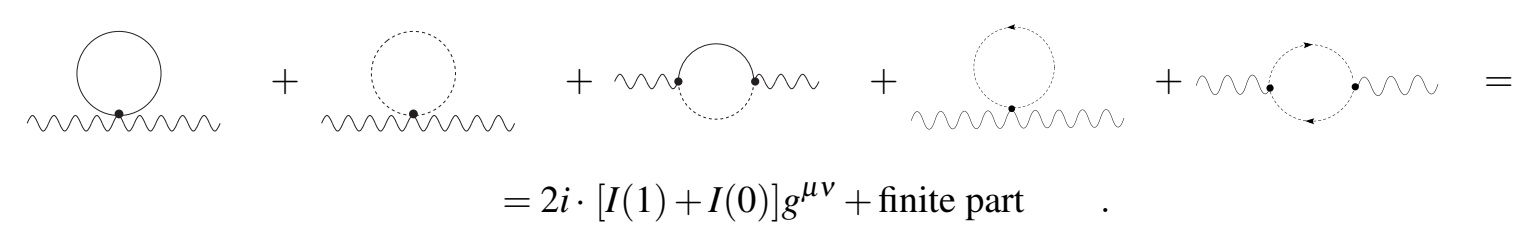

Note that the ultraviolet divergences come from integrals of the form:

$$
I\left(c^{2}\right)=\int \frac{d^{3} k}{(2 \pi)^{3}} \cdot \frac{i}{k^{2}-c^{2}+i \varepsilon},
$$

and that, unlike in $(1+1)$-dimensional scalar field theory, normal ordering is not enough. 


\begin{tabular}{cc}
\hline Diagram & Weight \\
\hline & $i\left[2\left(\kappa^{2}+1\right) I(1)+\kappa^{2} I(0)\right]$ \\
& $i\left[2\left(\kappa^{2}+1\right) I(1)+\kappa^{2} I(0)\right]$ \\
& $i\left[2\left(\kappa^{2}+1\right) I(1)+\kappa^{2} I(0)\right]$ \\
& $i\left[4\left(\kappa^{2}+1\right) I(1)+2 \kappa^{2} I(0)\right]$ \\
& $-2 i[I(1)+I(0)] g^{\mu \nu}$
\end{tabular}

Table 6: Counter-term vertices

We add the following counter-terms to cancel these divergences:

$$
\begin{aligned}
& \mathscr{L}_{\text {c.t. }}^{S}=\frac{\hbar}{2}\left[2\left(\kappa^{2}+1\right) \cdot I(1)+\kappa^{2} \cdot I(0)\right] \cdot\left[\Phi_{1}^{*}\left(x^{\mu}\right) \Phi_{1}\left(x^{\mu}\right)+\Phi_{2}^{*}\left(x^{\mu}\right) \Phi_{2}\left(x^{\mu}\right)-1\right] \\
& \mathscr{L}_{\text {c.t. }}^{A}=-\hbar[I(1)+I(0)] \cdot A_{\mu}\left(x^{\mu}\right) A^{\mu}\left(x^{\mu}\right)
\end{aligned}
$$

We have used a minimal subtraction prescription and the main criteria to set finite renormalizations have been: 1) The divergence due to the tadpole graph is exactly canceled in the self-dual limit $\kappa^{2}=1$. 2) The global $\mathbb{S U}(2)$ symmetry remains unbroken after one-loop renormalizations. Interested readers can find a fully detailed description of our renormalization conventions in [32].

\subsubsection{Mass renormalization counter-term energies}

Therefore, the topological soliton energy due to mass renormalization counter-terms in the self-dual limit $\kappa^{2}=1$ receives the following contribution from the scalar and vector fields:

$$
\Delta E_{C T}^{S}=\frac{\hbar m}{2}[4 I(1)+I(0)] \int d^{2} x\left(1-\left|S_{1}\right|^{2}-\left|S_{2}\right|^{2}\right), \quad \Delta E_{C T}^{A}=-\hbar m[I(1)+I(0)] \int d^{2} x V_{k} V_{k} .
$$

We reshuffle the sum of these two quantities into two pieces $\triangle E_{C T}^{\mathrm{I}(1)}=\frac{\hbar m}{2} I(1) \Sigma^{(1)}\left(S, V_{k}\right)$ and $\triangle E_{C T}^{\mathrm{I}(0)}=\frac{\hbar m}{2} I(0) \Sigma^{(0)}\left(S, V_{k}\right)$, respectively proportional to $I(1)$ and $I(0)$ :

$\Sigma^{(1)}\left(S, V_{k}\right)=4 \int d^{2} x\left(1-\left|S_{1}\right|^{2}-\left|S_{2}\right|^{2}-\frac{1}{2} V_{k} V_{k}\right), \Sigma^{(0)}\left(S, V_{k}\right)=\int d^{2} x\left(1-\left|S_{1}\right|^{2}-\left|S_{2}\right|^{2}-2 V_{k} V_{k}\right)$.

As in the zero point renormalization one must subtract the energy induced by the counter-terms in the vacuum from the same quantity for the topological soliton:

$$
\triangle E_{T S}^{R}=\triangle E_{C T}^{\mathrm{I}(1)}(T S)-\triangle E_{C T}^{\mathrm{I}(1)}(V)+\triangle E_{C T}^{\mathrm{I}(0)}(T S)-\triangle E_{C T}^{\mathrm{I}(0)}(V) .
$$

\subsection{High-temperature one-loop mass shift formula for self-dual semilocal topological solitons}

\subsubsection{Spectral zeta-function regularization}

Both $\triangle E_{T S}^{C}$ and $\triangle E_{T S}^{R}$ are divergent quantities that we shall regularize by means of the zeta function procedure before being added. We recall that the spectral zeta functions of elliptic operators $A$ (with positive definite and discrete spectrum) are formally defined as infinite sums of 
complex powers of their eigenvalues:

$$
\zeta_{A}(s)=\sum_{\operatorname{Spec} A} \lambda_{n}^{-s} \quad, \quad s \in \mathbb{C} \quad .
$$

These sums are usually convergent for $\operatorname{Re} s>s_{0}>0$, where $s_{0}$ is a positive real constant, but are susceptible to being analytically continued to the complex $s$-plane. In many favorable cases, their analytic continuations are meromorphic functions of $s$ and we shall regularize: 1) the ground state energy in topological sectors, 2) the ground state energy in the vacuum sector, and 3) the SSTS Casimir energy, by assigning to these quantities the values

$$
\begin{aligned}
\Delta E_{T S}(s) & =\frac{\hbar \mu}{2}\left(\frac{\mu^{2}}{m^{2}}\right)^{s}\left\{\zeta_{K}(s)-\zeta_{K^{G}}(s)\right\} & \Delta E_{0}(s) & =\frac{\hbar \mu}{2}\left(\frac{\mu^{2}}{m^{2}}\right)^{s}\left\{\zeta_{K_{0}}(s)-\zeta_{K_{0}^{G}}(s)\right\} \\
\Delta E_{T S}^{C}(s) & =\Delta E_{T S}(s)-\Delta E_{0}(s) & \Delta E_{T S}^{C} & =\lim _{s \rightarrow-\frac{1}{2}} \Delta M_{T S}^{C}(s)
\end{aligned}
$$

at a regular point of the spectral zeta functions in the complex $s$-plane. The spectral zeta functions of $K_{0}$ and $K_{0}^{G}$ with periodic boundary conditions on the edges of a square of area $l^{2}$ are given by meromorphic Euler Gamma functions:

$$
\zeta_{K_{0}}(s)=\frac{l^{2}}{\pi} \cdot \frac{\Gamma[s-1]}{\Gamma(s)}+\frac{l^{2}}{2 \pi} \cdot \frac{1}{(s-1) \Gamma(s)} \quad, \quad \zeta_{K_{0}^{G}}(s)=\frac{l^{2}}{4 \pi} \cdot \frac{\Gamma[s-1]}{\Gamma(s)}
$$

$\Delta E_{T S}^{R}$ can be regularized in a similar vein. On a square of area $l^{2} I(1)$ and $I(0)$ become infinite sums over discrete momenta:

$$
I(c)=\frac{1}{2} \int \frac{d^{2} k}{(2 \pi)^{2}} \frac{1}{\sqrt{\vec{k} \cdot \vec{k}+c^{2}}}=\frac{1}{2} \frac{1}{l^{2}} \sum_{\vec{k} \in \mathbb{Z}^{2}} \frac{1}{\sqrt{\vec{k} \cdot \vec{k}+c^{2}}} .
$$

Therefore, we regularize $\Delta E_{T S}^{R}$ in the same form:

$$
\Delta E_{T S}^{R}(s)=\frac{\hbar}{2 l^{2}}\left(\frac{\mu^{2}}{m^{2}}\right)^{s}\left(\zeta_{-\Delta+1}(s) \Sigma^{(1)}\left(S, V_{k}\right)+\zeta_{-\Delta}(s) \Sigma^{(0)}\left(S, V_{k}\right)\right) \quad, \quad \Delta E_{T S}^{R}=\lim _{s \rightarrow \frac{1}{2}} \Delta E_{T S}^{R}(s)
$$

knowing that:

$$
I(1)=\frac{1}{2 l^{2}} \zeta_{-\triangle+1}\left(\frac{1}{2}\right)=\frac{1}{8 \pi} \frac{\Gamma\left(-\frac{1}{2}\right)}{\Gamma\left(\frac{1}{2}\right)}=-\frac{1}{4 \pi} \quad, \quad I(0)=\frac{1}{2 l^{2}} \zeta_{-\triangle}\left(\frac{1}{2}\right)=-\frac{1}{4 \pi \sqrt{\pi}} .
$$

\subsubsection{The heat kernel expansion of elliptic differential operators}

Because the SSTS solutions are not known analytically, it is not possible to compute the spectral functions of $\zeta_{K}(s)$ and $\zeta_{K^{G}}(s)$. One possible loophole is to rely on the high-temperature asymptotic expansion of the heat traces and to build approximations to the spectral zeta functions from the Mellin transform of these approximated heat traces.

This construction is particularly complicated for the second-order fluctuation operator $K$. The Hessian is of the general form:

$$
K=K_{0}+Q_{k}(\vec{x}) \partial_{k}+V(\vec{x})
$$


The logical sequence is as follows: the $K$-heat equation kernel is the solution of the $K$-heat equation complying with the delta function infinite temperature condition:

$$
\left(\frac{\partial}{\partial \beta} \mathbb{I}+K\right) K_{K}(\vec{x}, \vec{y} ; \beta)=0 \quad, \quad K_{K}(\vec{x}, \vec{y} ; 0)=\mathbb{I} \cdot \delta^{(2)}(\vec{x}-\vec{y})
$$

In our problem $\mathbb{I}$ is the six $\times$ six unit matrix. The $K$-heat trace, or $K$-partition function, is the integral over the whole $\mathbb{R}^{2}$-plane of the $K$-heat equation kernel at the diagonal of $\mathbb{R}^{2} \otimes \mathbb{R}^{2}$, whereas the spectral $K$-zeta function is the Mellin transform of the $K$-heat trace:

$$
\operatorname{Tr} e^{-\beta K}=\operatorname{tr} \int_{\mathbb{R}^{2}} d^{2} \vec{x} K_{K}(\vec{x}, \vec{x} ; \beta) \quad, \quad \zeta_{K}(s)=\frac{1}{\Gamma(s)} \cdot \int_{0}^{\infty} d \beta \beta^{s-1} \operatorname{Tr} e^{-\beta K}
$$

Given the structure of $K$,

$$
K_{K}(\vec{x}, \vec{y} ; \beta)=C_{K}(\vec{x}, \vec{y} ; \beta) K_{K_{0}}(\vec{x}, \vec{y} ; \beta)
$$

is a good ansatz to solve (B.2I). Plugging the ansatz in (B.2J), one finds the transfer PDE and the high-temperature condition that $C(\vec{x}, \vec{y} ; \beta)$ must satisfy:

$$
\left\{\frac{\partial}{\partial \beta} \mathbb{I}+\frac{x_{k}-y_{k}}{\beta}\left(\partial_{k} \mathbb{I}-\frac{1}{2} Q_{k}\right)-\triangle \mathbb{I}+Q_{k} \partial_{k}+V\right\} C_{K}(\vec{x}, \vec{y} ; \beta)=0 \quad, \quad C_{K}(\vec{x}, \vec{y} ; 0)=\mathbb{I}
$$

The solution of (B.22) by means of a high-temperature power series expansion

$$
C_{K}(\vec{x}, \vec{y} ; \beta)=\sum_{n=0}^{\infty} c_{n}(\vec{x}, \vec{y} ; K) \beta^{n} \quad, \quad c_{0}(\vec{x}, \vec{y} ; K)=\mathbb{I}
$$

is tantamount to the solving of the recurrence relations:

$$
\left[n \mathbb{I}+\left(x_{k}-y_{k}\right)\left(\partial_{k} \mathbb{I}-\frac{1}{2} Q_{k}\right)\right] c_{n}(\vec{x}, \vec{y} ; K)=\left[\triangle \mathbb{I}-Q_{k} \partial_{k}-V\right] c_{n-1}(\vec{x}, \vec{y} ; K)
$$

It is easy to find the first Seeley density, $c_{1}(\vec{x}, \vec{x} ; K)=-V(\vec{x})$, a result used in the subsection above addressing the index theorem. Higher-order Seeley densities are harder to find. It is convenient to introduce the notation

$$
{ }^{\left(\alpha_{1}, \alpha_{2}\right)} C_{n}^{a b}(\vec{x})=\lim _{\vec{y} \rightarrow \vec{x}} \frac{\partial^{\alpha_{1}+\alpha_{2}}\left[c_{n}\right]_{a b}(\vec{x}, \vec{y} ; K)}{\partial x_{1}^{\alpha_{1}} \partial x_{2}^{\alpha_{2}}} \quad, \quad\left[c_{n}\right]_{a b}(\vec{x}, \vec{x} ; K)={ }^{(0,0)} C_{n}^{a b}(\vec{x})
$$

because the recurrence relations between derivatives of the Seeley densities can be written in the compact form 


$$
\begin{aligned}
& \left(k+\alpha_{1}+\alpha_{2}+1\right)^{\left(\alpha_{1}, \alpha_{2}\right)} C_{k+1}^{a b}(\vec{x})={ }^{\left(\alpha_{1}+2, \alpha_{2}\right)} C_{k}^{a b}(\vec{x})+{ }^{\left(\alpha_{1}, \alpha_{2}+2\right)} C_{k}^{a b}(\vec{x})- \\
& -\sum_{d=1}^{6} \sum_{r=0}^{\alpha_{1}} \sum_{t=0}^{\alpha_{2}}\left(\begin{array}{c}
\alpha_{1} \\
r
\end{array}\right)\left(\begin{array}{c}
\alpha_{2} \\
t
\end{array}\right)\left[\frac{\partial^{r+t} Q_{1}^{a d}}{\partial x_{1}^{r} \partial x_{2}^{t}}\left(\alpha_{1}-r+1, \alpha_{2}-t\right) C_{k}^{d b}(\vec{x})+\right. \\
& \left.+\frac{\partial^{r+t} Q_{2}^{a d}}{\partial x_{1}^{r} \partial x_{2}^{t}}\left(\alpha_{1}-r, \alpha_{2}-t+1\right) C_{k}^{d b}(\vec{x})\right]+ \\
& +\frac{1}{2} \sum_{d=1}^{6} \sum_{r=0}^{\alpha_{1}-1} \sum_{t=0}^{\alpha_{2}} \alpha_{1}\left(\begin{array}{c}
\alpha_{1}-1 \\
r
\end{array}\right)\left(\begin{array}{c}
\alpha_{2} \\
t
\end{array}\right) \frac{\partial^{r+t} Q_{1}^{a d}}{\partial x_{1}^{r} \partial x_{2}^{t}}\left(\alpha_{1}-1-r, \alpha_{2}-t\right) C_{k+1}^{d b}(\vec{x})+ \\
& +\frac{1}{2} \sum_{d=1}^{6} \sum_{r=0}^{\alpha_{2}-1} \sum_{t=0}^{\alpha_{1}} \alpha_{2}\left(\begin{array}{c}
\alpha_{2}-1 \\
r
\end{array}\right)\left(\begin{array}{c}
\alpha_{1} \\
t
\end{array}\right) \frac{\partial^{r+t} Q_{2}^{a d}}{\partial x_{1}^{t} \partial x_{2}^{r}}\left(\alpha_{1}-t, \alpha_{2}-1-r\right) C_{k+1}^{d b}(\vec{x})- \\
& -\sum_{d=1}^{6} \sum_{r=0}^{\alpha_{2}} \sum_{t=0}^{\alpha_{1}}\left(\begin{array}{c}
\alpha_{1} \\
t
\end{array}\right)\left(\begin{array}{c}
\alpha_{2} \\
r
\end{array}\right) \frac{\partial^{r+t} V^{a d}}{\partial x_{1}^{t} \partial x_{2}^{r}}\left(\alpha_{1}-t, \alpha_{2}-r\right) C_{k}^{d b}(\vec{x}) \\
& c_{0}(\vec{x}, \vec{x} ; K)=\mathbb{I} \Rightarrow\left\{\begin{array}{c}
{ }^{(\alpha, \beta)} C_{0}^{a b}(\vec{x})=0, \text { if } \alpha \neq 0, \text { and } / \text { or } \beta \neq 0 \\
{ }^{(0,0)} C_{0}^{a a}(\vec{x})=1, a=1,2, \cdots, 6
\end{array}\right.
\end{aligned}
$$

susceptible to being solved with the help of a symbolic programm implemented in Mathematica.

\subsubsection{Mellin transform of the heat trace asymptotic expansion}

The spectral zeta functions of of both $K$ and $K^{G}$ are obtained from the high-temperature expansion of the heat traces via the Mellin transform:

$$
\begin{aligned}
\zeta_{K}(s) & =\frac{1}{\Gamma(s)} \int_{0}^{1} d \beta \beta^{s-1}\left\{\frac{1}{4 \pi \beta} \sum_{n=0}^{\infty} \beta^{n} \cdot\left(e^{-\beta} \sum_{a=1}^{4}\left[c_{n}^{I}(K)\right]_{a a}+\sum_{a=5}^{6}\left[c_{n}^{O}(K)\right]_{a a}\right)\right\} \\
& +\frac{1}{\Gamma(s)} \int_{1}^{\infty} d \beta \beta^{s-1} \operatorname{Tr} e^{-\beta K} \\
\zeta_{K^{G}}(s) & =\frac{1}{\Gamma(s)} \int_{0}^{1} d \beta \beta^{s-1}\left\{\frac{e^{-\beta}}{4 \pi \beta} \sum_{n=0}^{\infty} \beta^{n} c_{n}\left(K^{\mathrm{G}}\right)\right\}+\frac{1}{\Gamma(s)} \int_{1}^{\infty} d \beta \beta^{s-1} \operatorname{Tr} e^{-\beta K^{G}}
\end{aligned}
$$

Here the Seeley coefficients $\left[c_{n}^{I}(K)\right]_{a a},\left[c_{n}^{O}(K)\right]_{a a}$, and $c_{n}\left(K^{G}\right)$ are obtained through integration over the whole $\mathbb{R}^{2}$-plane of the Seeley densities:

$$
\begin{aligned}
{\left[c_{n}^{I}(K)\right]_{a a} } & =\int d^{2} x\left[c_{n}\right]_{a a}(\vec{x}, \vec{x} ; K) \quad, \quad a=1,2,3,4 \\
{\left[c_{n}^{O}(K)\right]_{a a} } & =\int d^{2} x\left[c_{n}\right]_{a a}(\vec{x}, \vec{x} ; K) \quad, \quad a=5,6 \quad, \quad\left[c_{n}\left(K^{G}\right)\right]=\int d^{2} x\left[c_{n}\right]\left(\vec{x}, \vec{x} ; K^{G}\right) .
\end{aligned}
$$

Finally, we find the spectral zeta functions as sums of meromorphic and entire parts:

$$
\begin{aligned}
\zeta_{K}(s) & =\sum_{n=0}^{\infty}\left\{\sum_{a=1}^{4}\left[c_{n}^{I}(K)\right]_{a a} \frac{\gamma[s+n-1,1]}{4 \pi \Gamma(s)}+\sum_{a=5}^{6}\left[c_{n}^{O}(K)\right]_{a a} \frac{1}{4 \pi \Gamma(s)(s+n-1)}\right\}+\frac{1}{\Gamma(s)} B_{K}(s) \\
\zeta_{K^{G}}(s) & =\sum_{n=0}^{\infty} c_{n}\left(K^{G}\right) \frac{\gamma[s+n-1,1]}{4 \pi \Gamma(s)}+\frac{1}{\Gamma(s)} B_{K^{G}}(s) .
\end{aligned}
$$


An important warning: the physical point $s=-\frac{1}{2}$ is a regular point -not a pole- of $\zeta_{K}(s)$, $\zeta_{K^{G}}(s), \zeta_{K_{0}}(s)$, and $\zeta_{K_{0}^{G}}(s)$. Unlike in kink cases, after zeta function regularization is performed only finite renormalizations are left in $(2+1)$-dimensional field theories.

\subsubsection{High-temperature one-loop mass shift formula for self-dual semi-local topological solitons}

Neglecting the entire parts and truncating the zeta functions at a finite number of summands $N_{0}$, the high-temperature one-loop formula giving the semi-classical shift to the masses of semilocal self-dual topological solitons is obtained:

$$
\begin{aligned}
\Delta M_{T S} & =\triangle E_{T S}^{C}+\triangle E_{T s}^{R}= \\
& =-\frac{\hbar m}{16 \pi \sqrt{\pi}}\left[\sum_{n=2}^{N_{0}}\left\{\left[\sum_{a=1}^{4}\left[c_{n}(K)\right]_{a a}-c_{n}\left(K^{\mathrm{G}}\right)\right] \cdot \gamma\left[n-\frac{3}{2}, 1\right]+\sum_{a=5}^{6} \frac{\left[c_{n}(K)\right]_{a a}}{n-\frac{3}{2}}\right\}+4 l \cdot 8 \pi\right] \\
& -\frac{\hbar m}{8 \pi \sqrt{\pi}} \cdot \int d^{2} x\left|S_{2}\right|^{2}\left(x_{1}, x_{2}\right) \cdot\left(\gamma\left[-\frac{1}{2}, 1\right]-2\right)
\end{aligned}
$$

The following remarks are meaningful:

1. The factor $-2 l \frac{\hbar m}{\sqrt{\pi}}$ is due to the subtraction of the $4 l$ zero modes by exactly the same procedure as the regularization method used for kinks.

2. Unlike in theories with only massive particles, the criterion of the exact cancelation of tadpole graphs is not completely equivalent to the cancelation of the contributions of the first-order diagonal Seeley coefficient. The term in the second row of formula (3.25) is the mismatch between these two criteria due to the massless particles in the semi-local Abelian Higgs model.

3. The ANO vortices correspond to the SSTS solitons with $S_{2}(\vec{x})=0$. Freezing the $\delta S_{2}\left(x_{0}, \vec{x}\right)$ fluctuations and dropping away the associated $2 l$ zero modes, the one-loop mass shifts for ANO vortices are attained.

\subsection{Mathematica calculations}

In this last Section we shall solve the recurrence relations to find the Seeley densities and their associated Seeley coefficients by a mixture of symbolic and numerical Programs implemented in Mathematica. We shall focus on circle symmetric SSTS solutions,

$$
\begin{array}{llrl}
S_{1}^{1}\left(x_{1}, x_{2}\right) & =f(r) \cos \theta & & S_{1}^{2}\left(x_{1}, x_{2}\right)=f(r) \sin \theta \\
S_{2}^{1}\left(x_{1}, x_{2}\right) & =h(r) & & S_{2}^{2}\left(x_{1}, x_{2}\right)=0 \\
V_{1}\left(x_{1}, x_{2}\right) & =-\frac{\alpha(r)}{r} \sin \theta & & V_{2}\left(x_{1}, x_{2}\right)=\frac{\alpha(r)}{r} \cos \theta
\end{array}
$$

the only ones at our disposal, although $f(r), h(r)$, and $\alpha(r)$ have been found numerically. 


\subsubsection{Seeley densities for circle symmetric semi-local vortices}

The first step is to use the first-order rotationally symmetric ODE (B.7) to write the field derivatives in terms of the field profiles themselves:

$$
\begin{array}{lrrr}
\frac{\partial S_{1}^{1}}{\partial x_{1}}=\frac{f(r)}{r}\left[1-\alpha(r) \cos ^{2} \theta\right] & \frac{\partial S_{1}^{2}}{\partial x_{2}}=\frac{f(r)}{r}\left[1-\alpha(r) \sin ^{2} \theta\right] & \frac{\partial S_{2}^{1}}{\partial x_{2}}=-\frac{h(r)}{r} \alpha(r) \cos \theta \\
\frac{\partial S_{1}^{1}}{\partial x_{2}}=-\frac{f(r)}{r} \alpha(r) \cos \theta \sin \theta & \left.\frac{\partial S_{1}^{2}}{\partial x_{1}}=-\frac{f(r)}{r} \alpha(r)\right) \sin \theta \cos \theta & \left.\frac{\partial S_{2}^{1}}{\partial x_{1}}=-\frac{h(r)}{r} \alpha(r)\right) \sin \theta \\
\frac{\partial V_{1}}{\partial x_{1}}=\frac{\cos 2 \theta}{2}\left[\frac{2 f(r) \alpha(r)}{r}+\frac{f^{2}(r)+h^{2}(r)-1}{2}\right] & \frac{\partial V_{1}}{\partial x_{2}}=-\cos 2 \theta \frac{\alpha(r)}{r^{2}}+\frac{\sin ^{2} \theta}{2}\left(f^{2}(r)+h^{2}(r)-1\right) \\
\frac{\partial V_{2}}{\partial x_{1}}=-\cos 2 \theta \frac{\alpha(r)}{r^{2}}-\frac{\cos ^{2} \theta}{2}\left(f^{2}(r)+h^{2}(r)-1\right) & \frac{\partial V_{2}}{\partial x_{2}}=-\frac{\cos 2 \theta}{2}\left[\frac{2 f(r) \alpha(r)}{r}+\frac{f^{2}(r)+h^{2}(r)-1}{2}\right] .
\end{array}
$$

This step is important because numerical calculations on field derivatives cause considerable errors. The next step is the Mathematica solution of the rotationally symmetric recurrence relations. Below we list the three first-order circle symmetric Seeley densities:

$$
\begin{aligned}
& \operatorname{tr} c_{1}^{I}(r)=5-\frac{2 \alpha(r)^{2}}{r^{2}}-5 f(r)^{2}-3 h(r)^{2} \\
& \begin{aligned}
\operatorname{tr} c_{2}^{I}(r)=\frac{1}{12 r^{4}}[ & 4 \alpha(r)^{4}+27 r^{4} f(r)^{4}-8 r^{2} \alpha(r)\left(-1+14 f(r)^{2}+h(r)^{2}\right)+ \\
+ & 8 \alpha(r)^{2}\left(-2-3 r^{2}+9 r^{2} f(r)^{2}+3 r^{2} h(r)^{2}\right)+ \\
& \left.+f(r)^{2}\left(56 r^{2}-64 r^{4}+34 r^{4} h(r)^{2}\right)+r^{4}\left(37-32 h(r)^{2}+7 h(r)^{4}\right)\right] \\
\operatorname{tr} c_{3}^{I}(r)=\frac{1}{120 r^{6}}\{ & -4 \alpha(r)^{6}-4 r^{2} \alpha(r)^{3}\left(14+35 f(r)^{2}-36 h(r)^{2}\right)+ \\
+ & 4 \alpha(r)^{4}\left(20+9 r^{2}+32 r^{2} f(r)^{2}+26 r^{2} h(r)^{2}\right)- \\
-2 r^{2} \alpha(r)\left[57 r^{2} f(r)^{4}+f(r)^{2}\left(32+331 r^{2}-75 r^{2} h(r)^{2}\right)-4\left(-1+h(r)^{2}\right)\left(-16-9 r^{2}+r^{2} h(r)^{2}\right)\right]+ & \\
+ & \alpha(r)^{2}\left[-256-144 r^{2}-117 r^{4}+99 r^{4} f(r)^{4}-16 r^{2} h(r)^{2}+94 r^{4} h(r)^{2}-61 r^{4} h(r)^{4}+\right. \\
\quad & \left.2 r^{2} f(r)^{2}\left(56+183 r^{2}+19 r^{2} h(r)^{2}\right)\right]+ \\
+ & r^{4}\left[-16+151 r^{2}-29 r^{2} f(r)^{6}+\left(32-135 r^{2}\right) h(r)^{2}+\left(-16+23 r^{2}\right) h(r)^{4}+r^{2} h(r)^{6}+\right. \\
+f(r)^{4} & \left.\left.\left(-20+199 r^{2}-57 r^{2} h(r)^{2}\right)+f(r)^{2}\left(392-321 r^{2}+2\left(-68+111 r^{2}\right) h(r)^{2}-27 r^{2} h(r)^{4}\right)\right]\right\} .
\end{aligned}
\end{aligned}
$$

Here, tr means that we have summed up to the fourth diagonal density.

$$
\begin{aligned}
& \operatorname{tr} c_{1}^{O}(r)=1-\frac{2 \alpha(r)^{2}}{r^{2}}-f(r)^{2}-3 h(r)^{2} \\
& \begin{aligned}
\operatorname{tr} c_{2}^{O}(r)=\frac{1}{12 r^{4}}[ & \alpha \alpha(r)^{4}-r^{4} f(r)^{4}+8 r^{2} \alpha(r)\left(1+2 f(r)^{2}-h(r)^{2}\right)- \\
& -8 \alpha(r)^{2}\left(2+r^{2}+r^{2} f(r)^{2}-5 r^{2} h(r)^{2}\right)+2 r^{2} f(r)^{2}\left(-4+9 r^{2} h(r)^{2}\right)+ \\
& \left.+r^{4}\left(1-8 h(r)^{2}+19 h(r)^{4}\right)\right]
\end{aligned}
\end{aligned}
$$




$$
\begin{aligned}
& \operatorname{tr} c_{3}^{O}(r)=\frac{-1}{120 r^{6}}\left\{4 \alpha(r)^{6}-4 r^{2} \alpha(r)^{3}\left(-14+9 f(r)^{2}+84 h(r)^{2}\right)-\right. \\
&-4 \alpha(r)^{4}\left(20+3 r^{2}+2 r^{2}\left(f(r)^{2}+4 h(r)^{2}\right)\right)+ \\
&+\alpha(r)^{2}\left[256+48 r^{2}-3 r^{4}+45 r^{4} f(r)^{4}+2 r^{2}\left(-40+89 r^{2}\right) h(r)^{2}-115 r^{4} h(r)^{4}+\right. \\
&\left.\quad+2 r^{2} f(r)^{2}\left(8+5 r^{2}-35 r^{2} h(r)^{2}\right)\right]- \\
&-2 r^{2} \alpha(r)\left[53 r^{2} f(r)^{4}+4\left(-1+h(r)^{2}\right)\left(-16-3 r^{2}+7 r^{2} h(r)^{2}\right)-f(r)^{2}\left(32+17 r^{2}+47 r^{2} h(r)^{2}\right)\right]+ \\
&+r^{4}\left[16+3 r^{2}+3 r^{2} f(r)^{6}-\left(32+19 r^{2}\right) h(r)^{2}+\left(16+23 r^{2}\right) h(r)^{4}+33 r^{2} h(r)^{6}+\right. \\
&\left.\left.\quad+f(r)^{4}\left(52-r^{2}+39 r^{2} h(r)^{2}\right)+f(r)^{2}\left(-24-5 r^{2}+\left(-72+22 r^{2}\right) h(r)^{2}+69 r^{2} h(r)^{4}\right)\right]\right\} .
\end{aligned}
$$

tr now means that we have summed $\left[c_{n}^{O}\right]_{55}$ and $\left[c_{n}^{O}\right]_{66}$.

$$
\begin{aligned}
& c_{1}^{G}(r)=1-f(r)^{2}-h(r)^{2} \\
& c_{2}^{G}(r)=\frac{1}{6 r^{2}}[ 3 r^{2}+2 r^{2} f(r)^{4}-\left(5 r^{2}+4 \alpha(r)^{2}\right) h(r)^{2}+2 r^{2} h(r)^{4}+ \\
&\left.+f(r)^{2}\left(-4-5 r^{2}+8 \alpha(r)-4 \alpha(r)^{2}+4 r^{2} h(r)^{2}\right)\right] \\
& c_{3}^{G}(r)=\frac{-1}{60 r^{4}}\{-10 r^{4}+4 r^{4} f(r)^{6}+\left[23 r^{4}-8 r^{2} \alpha(r)+16\left(1+r^{2}\right) \alpha(r)^{2}+32 \alpha(r)^{3}+16 \alpha(r)^{4}\right] h(r)^{2}+ \\
&+r^{2}\left[-17 r^{2}+8 \alpha(r)-16 \alpha(r)^{2}\right] h(r)^{4}+4 r^{4} h(r)^{6}+ \\
&+r^{2} f(r)^{4}\left[-24-17 r^{2}+40 \alpha(r)-16 \alpha(r)^{2}+12 r^{2} h(r)^{2}\right]+ \\
&+ f(r)^{2}\left[-32 \alpha(r)^{3}+16 \alpha(r)^{4}+8 r^{2} \alpha(r)\left(-5+6 h(r)^{2}\right)+16 \alpha(r)^{2}\left(1+r^{2}-2 r^{2} h(r)^{2}\right)+\right. \\
&\left.\left.+r^{2}\left(24+23 r^{2}-2\left(10+17 r^{2}\right) h(r)^{2}+12 r^{2} h(r)^{4}\right)\right]\right\} .
\end{aligned}
$$

\subsubsection{One-loop SSTS mass shifts}

In this sub Section we offer some Tables with Mathematica calculations of the one-loop corrections to the masses of topological solitons in the semi-local Abelian Higgs model. We denote the Seeley coefficients, calculated by means of numerical integration of the circle symmetric Seeley densities evaluated at the numerical solutions for the field profiles, in the form:

$$
\operatorname{tr} c_{n}^{I}=2 \pi \int_{0}^{\infty} d r r \operatorname{tr} c_{n}^{I}(r) \quad, \quad \operatorname{tr} c_{n}^{O}=2 \pi \int_{0}^{\infty} d r r \operatorname{tr} c_{n}^{O}(r) \quad, \quad c_{n}^{G}=2 \pi \int_{0}^{\infty} d r r c_{n}^{G}(r) \quad .
$$

First, we focus on the embedded ANO vortices, the $h_{0}$ topological solitons. Second, we give results for several values of $h_{0}$ up to a value of $h_{0}$ close to $h_{0}=1$, which corresponds to the $\mathbb{C P}^{1}$-lumps. 
1. One-loop vortex mass shifts in units of $\hbar m$ :

Because the ANO vortex solutions were generated numerically, integration over the whole plane of the Seeley densities can also only be performed numerically. Therefore, we are forced to put a cut-off in the area and replace the infinite plane by a discus of radius $R$, which in the calculations shown below was chosen to be $R=10.000$

\begin{tabular}{|c|c|c|c|}
\hline & \multicolumn{3}{|c|}{$h_{0}=0.0$} \\
\hline$n$ & $\operatorname{tr} c_{n}^{I}$ & $\operatorname{tr} c_{n}^{O}$ & $c_{n}^{G}$ \\
\hline 1 & -41.4469 & -91.8429 & 12.599 \\
\hline 2 & 30.3736 & 0.96286 & 2.61518 \\
\hline 3 & 12.9447 & -0.0592415 & 0.32005 \\
\hline 4 & 4.22603 & 0.001512548 & 0.0230445 \\
\hline 5 & 1.05059 & 0.000758663 & 0.0013023 \\
\hline 6 & 0.20900 & -0.00023912 & 0.0000698185 \\
\hline
\end{tabular}

\begin{tabular}{|c|c|}
\hline$N_{0}$ & $\Delta M_{V}\left(N_{0}\right)$ \\
& $l=1$ \\
\hline 2 & -1.61536 \\
3 & -1.66862 \\
4 & -1.67809 \\
5 & -1.67966 \\
6 & -1.67989 \\
\hline
\end{tabular}

Table 7: Seeley coefficients for the (left) and Quantum Mass Correction (right) to the soliton in the semilocal Abelian Higgs model with $h_{0}=0.0$.

In the Table on the left, the first six Seeley coefficients are given. The next coefficients are very small and one expects that the approximation to the exact value of the one-loop mass shift is quite good, as shown in the Table on the right: the mass shift obtained by counting five or six coefficients agrees up to the third decimal figure. In fact, the thresholds in the wells are $\leq 1.1$ is the expectation value of the scalar field at the vacuum, and we find a similar situation to sine-Gordon kinks (see the first Part), where keeping six coefficients provides a fairly good approximation.

From the same Table one can read the one-loop mass shifts of ANO vortices with $l=1$ in the Abelian Higgs model: just take $\operatorname{tr} c_{n}^{O}(K)$ equal to zero and subtract two zero modes, not 4 . The ratio is:

$$
\frac{\Delta M_{V}^{\mathrm{SLAHM}}}{\Delta M_{V}^{\mathrm{AHM}}}=\frac{1.67989}{1.09449}=1.53486
$$

A similar proportion exists between the ratios of kink mass shifts in the $\lambda(\phi)_{2}^{4}$ model and the BNRT model, both treated in the first Part:

$\frac{\Delta M_{K}^{\mathrm{BNRTM}}}{\Delta M_{K}^{\lambda \Phi \mathrm{M}}}=\frac{0.693943}{0.471113}=1.47299, \sigma=0.99 \quad, \quad \frac{\Delta M_{K}^{\mathrm{BNRTM}}}{\Delta M_{K}^{\lambda \Phi \mathrm{M}}}=\frac{0.698445}{0.471113}=1.48254, \sigma=1.01$.

\section{SSTS one-loop mass shifts}

Whereas the one-loop mass shift of ANO embedded vortices is always negative and varies extremely slowly as the area increases towards more negative values, one-loop mass shifts of genuine semilocal topological solitons with $\left|h_{0}\right|>0$ become less negative, and even positive, for larger areas, as is shown in the following Table.

The classical degeneracy in energy between semi-local topological defects seems to be broken by one-loop fluctuations, the ANO embedded vortices becoming the ground states in the topological sector of one quantum of magnetic flux. It is remarkable how strong this effect becomes for topological solitons close to $\mathbb{C P}^{1}$-lumps, $\left|h_{0}\right| \simeq 1$. 


\begin{tabular}{|c|c|c|c|c|c|}
\hline$R$ & $\Delta M_{V}\left(N_{0}=6, R\right)$ & $\Delta M_{V}\left(N_{0}=6, R\right)$ & $\Delta M_{V}\left(N_{0}=6, R\right)$ & $\Delta M_{V}\left(N_{0}=6, R\right)$ & $\Delta M_{V}\left(N_{0}=6, R\right)$ \\
& $h_{0}=0.0$ & $h_{0}=0.1$ & $h_{0}=0.3$ & $h_{0}=0.6$ & $h_{0}=0.9$ \\
\hline $10^{2}$ & -1.67955 & -1.61672 & -1.05000 & 2.10142 & 24.6066 \\
$10^{3}$ & -1.67971 & -1.58311 & -0.626167 & 4.5485 & 42.7747 \\
$10^{4}$ & -1.67989 & -1.55133 & -0.252586 & 6.41655 & 60.9433 \\
$10^{5}$ & -1.68005 & -1.51957 & 0.12086 & 8.5741 & 79.1116 \\
$10^{6}$ & -1.68026 & -1.48779 & 0.49433 & 10.7203 & 97.2798 \\
\hline
\end{tabular}

Table 8: One-loop mass shifts for semi-local topological solitons: Five values of $h_{0}$, five values of $R$, and fixed $N_{0}=6$.

\subsubsection{Infrared divergences: quantum fate of semi-local topological solitons}

The origin of the degeneracy breaking is the slow decay (non-exponential) to their vacuum values of genuine semilocal topological solitons as compared with ANO vortices. Plugging the asymptotic form of the circle symmetric topological soliton solutions in the Seeley densities, we find the following behavior at infinity in terms of the parameter $\left|h^{1}\right|$ (which sets the long $r$ behavior of the solutions):

$$
\begin{aligned}
& 2 \pi r \operatorname{tr} c_{1}^{I}(r) \stackrel{r \rightarrow \infty}{\simeq}-\frac{4 \pi}{r}\left(1-\left|h^{1}\right|^{2}\right)+\frac{4 \pi}{r^{3}}\left(12\left|h^{1}\right|^{2}-\left|h^{1}\right|^{4}\right)+\mathscr{O}\left(\frac{1}{r^{5}}\right) \\
& 2 \pi r \operatorname{tr} c_{1}^{O}(r) \stackrel{r \rightarrow \infty}{\simeq}-\frac{4 \pi}{r}\left(1+\left|h^{1}\right|^{2}\right)+\frac{4 \pi}{r^{3}}\left(4\left|h^{1}\right|^{2}+\left|h^{1}\right|^{4}\right)+\mathscr{O}\left(\frac{1}{r^{5}}\right) \\
& 2 \pi r c_{1}^{G}(r) \stackrel{r \rightarrow \infty}{\simeq} \frac{8 \pi}{r^{3}}\left|h^{1}\right|^{2}+\mathscr{O}\left(\frac{1}{r^{5}}\right) \\
& 2 \pi r \operatorname{tr} c_{2}^{I}(r) \stackrel{r \rightarrow \infty}{\simeq} \frac{2 \pi}{r}\left|h^{1}\right|^{2}+\frac{2 \pi}{r^{3}}\left(-1+4\left|h^{1}\right|^{2}-\left|h^{1}\right|^{4}\right)+\mathscr{O}\left(\frac{1}{r^{5}}\right) \\
& 2 \pi r \operatorname{tr} c_{2}^{O}(r) \stackrel{r \rightarrow \infty}{\simeq} \frac{2 \pi}{r}\left|h^{1}\right|^{2}+\frac{2 \pi}{r^{3}}\left(-1+4\left|h^{1}\right|^{2}-\left|h^{1}\right|^{4}\right)+\mathscr{O}\left(\frac{1}{r^{5}}\right) \\
& 2 \pi r c_{2}^{G}(r) \stackrel{r \rightarrow \infty}{\simeq} \frac{64 \pi}{3 r^{5}}\left|h^{1}\right|^{2}+\frac{\pi}{r^{7}}\left(768\left|h^{1}\right|^{2}-80\left|h^{1}\right|^{4}\right)+\mathscr{O}\left(\frac{1}{r^{9}}\right) \\
& 2 \pi r \operatorname{tr} c_{3}^{I}(r) \stackrel{r \rightarrow \infty}{\simeq} \frac{2 \pi}{3 r}\left|h^{1}\right|^{2}+\frac{2 \pi}{3 r^{3}}\left(3\left|h^{1}\right|^{2}-\left|h^{1}\right|^{4}\right)+\mathscr{O}\left(\frac{1}{r^{5}}\right) \\
& 2 \pi r \operatorname{tr} c_{3}^{O}(r) \stackrel{r \rightarrow \infty}{\simeq}-\frac{2 \pi^{2}}{3 r}\left|h^{1}\right|^{2}+\frac{2 \pi}{3 r^{3}}\left(-4\left|h^{1}\right|^{2}+\left|h^{1}\right|^{4}\right)+\mathscr{O}\left(\frac{1}{r^{5}}\right) \\
& 2 \pi r c_{3}^{G}(r) \stackrel{r \rightarrow \infty}{\simeq} \frac{384 \pi}{5 r^{7}}\left|h^{1}\right|^{2}+\mathscr{O}\left(\frac{1}{r^{8}}\right) \\
& 2 \pi r \operatorname{tr} c_{4}^{I}(r) \stackrel{r \rightarrow \infty}{\simeq} \frac{\pi}{6 r}\left|h^{1}\right|^{2}+\frac{\pi}{6 r^{3}}\left(\frac{12}{5}\left|h^{1}\right|^{2}-\left|h^{1}\right|^{4}\right)+\mathscr{O}\left(\frac{1}{r^{5}}\right) \\
& 2 \pi r \operatorname{tr} c_{4}^{O}(r) \stackrel{r \rightarrow \infty}{\simeq}-\frac{\pi}{6 r}\left|h^{1}\right|^{2}+\frac{\pi}{6 r^{3}}\left(4\left|h^{1}\right|^{2}-\left|h^{1}\right|^{4}\right)+\mathscr{O}\left(\frac{1}{r^{5}}\right) \\
& 2 \pi r c_{4}^{G}(r) \stackrel{r \rightarrow \infty}{\simeq} \frac{832 \pi}{21 r^{9}}\left|h^{1}\right|^{4}-\frac{43 \pi}{30 r^{9}}\left|h^{1}\right|^{6}+\frac{\pi}{48 r^{9}}\left|h^{1}\right|^{8}+\frac{\pi}{768 r^{9}}\left|h^{1}\right|^{10}+\mathscr{O}\left(\frac{1}{r^{11}}\right) \\
& 2 \pi r \operatorname{trc} c_{5}^{I}(r) \stackrel{r \rightarrow \infty}{\simeq} \frac{\pi}{30 r}\left|h^{1}\right|^{2}+\frac{\pi}{30 r^{3}}\left(2\left|h^{1}\right|^{2}-\left|h^{1}\right|^{4}\right)+\mathscr{O}\left(\frac{1}{r^{5}}\right)
\end{aligned}
$$




$$
\begin{aligned}
2 \pi r \operatorname{trc} c_{5}^{O}(r) \stackrel{r \rightarrow \infty}{\simeq}-\frac{\pi}{30 r}\left|h^{1}\right|^{2}+\frac{\pi}{30 r^{3}}\left(-4\left|h^{1}\right|^{2}+\left|h^{1}\right|^{4}\right)+\mathscr{O}\left(\frac{1}{r^{5}}\right) \\
2 \pi r c_{5}^{G}(r) \stackrel{r \rightarrow \infty}{\simeq}-\frac{\pi}{r^{9}}\left(\frac{2048}{105}\left|h^{1}\right|^{2}-\frac{416}{189}\left|h^{1}\right|^{4}+\frac{43}{540}\left|h^{1}\right|^{6}-\frac{1}{864}\left|h^{1}\right|^{8}+\frac{1}{138240}\left|h^{1}\right|^{10}\right)+\mathscr{O}\left(\frac{1}{r^{11}}\right) \\
2 \pi r \operatorname{trc} c_{6}^{I}(r) \stackrel{r \rightarrow \infty}{\simeq} \frac{\pi}{180 r}\left|h^{1}\right|^{2}+\frac{\pi}{15 r^{3}}\left(\frac{1}{7}\left|h^{1}\right|^{2}-\frac{1}{12}\left|h^{1}\right|^{4}\right)+\mathscr{O}\left(\frac{1}{r^{5}}\right) \\
2 \pi r \operatorname{trc} c_{6}^{O}(r) \stackrel{r \rightarrow \infty}{\simeq} \frac{\pi}{180 r}\left|h^{1}\right|^{2}+\frac{\pi}{45 r^{3}}\left(\left|h^{1}\right|^{2}-\frac{1}{4}\left|h^{1}\right|^{4}\right)+\mathscr{O}\left(\frac{1}{r^{5}}\right)
\end{aligned}
$$

The key observation is the appearance of infrared logarithmic divergences in the Seeley coefficients $\operatorname{tr} c_{n}^{I}(K)$ and $\operatorname{tr} c_{n}^{O}(K)$ for all $n$. The ghost coefficients $c_{n}^{G}\left(K^{G}\right)$, however, are infrared convergent. The combination of the signs that we have seen in the previous sub-sections with the long $r$ behavior shows that one-loop mass shifts of semi-local topological solitons tend to $+\infty$ in the infinite area limit. Semi-local topological defects grow infinitely massive due to the infrared effects of one-loop fluctuations. This phenomenon seems to be amazingly close to the non-existence of Goldstone bosons in (1+1)-dimensions.

There is a very important exception: for ANO vortices, $\left|h^{1}\right|=0$ and only the first-order coefficients are infrared divergent. However, the contribution of these coefficients is totally canceled by mass renormalization counter-terms. Our results suggest that only the ANO vortices between all the semi-local topological solitons survive one-loop quantum fluctuations. It would be very interesting to try a more analytic approach to this problem in order to fully elucidate this delicate issue.

\subsubsection{Circle symmetric self-dual Abrikosov-Nielsen-Olesen vortices: one-loop mass shifts up to $l=4$}

Finally, we consider the problem of computing the one-loop mass shifts for superimposed ANO vortices at the $\kappa=1$ limit up to four quanta $-8 \pi$ - of magnetic flux in the Abelian Higgs model (no fluctuations in the $a=5,6$ directions and $2 l$ zero modes). The Figure 13 shows the
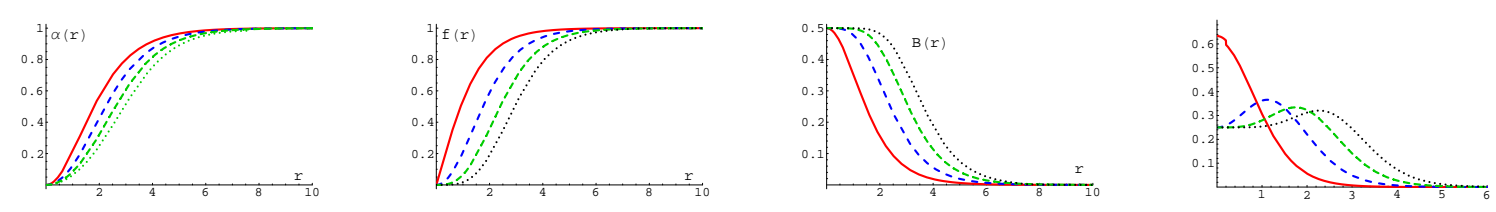

Figure 13: Plots of the field profiles $\alpha(r)$ (a) and $f(r)$ (b), the magnetic field $B(r)$ (c), and the energy density $\varepsilon(r)$ for self-dual vortices with $l=1$ (solid line), $l=2$ (broken line), $l=3$ (broken-dotted line) and $l=4$ (dotted line).

field profiles, the magnetic field, and the energy density for the numerically generated solutions for $l=1, l=2, l=3$, and $l=4$ in [2G]. The Figure 14, however, encompass the 3D plots of these solutions.

The Seeley coefficients for the different values of $l$ as well as the one-loop mass shifts are displayed in the Tables below 4 

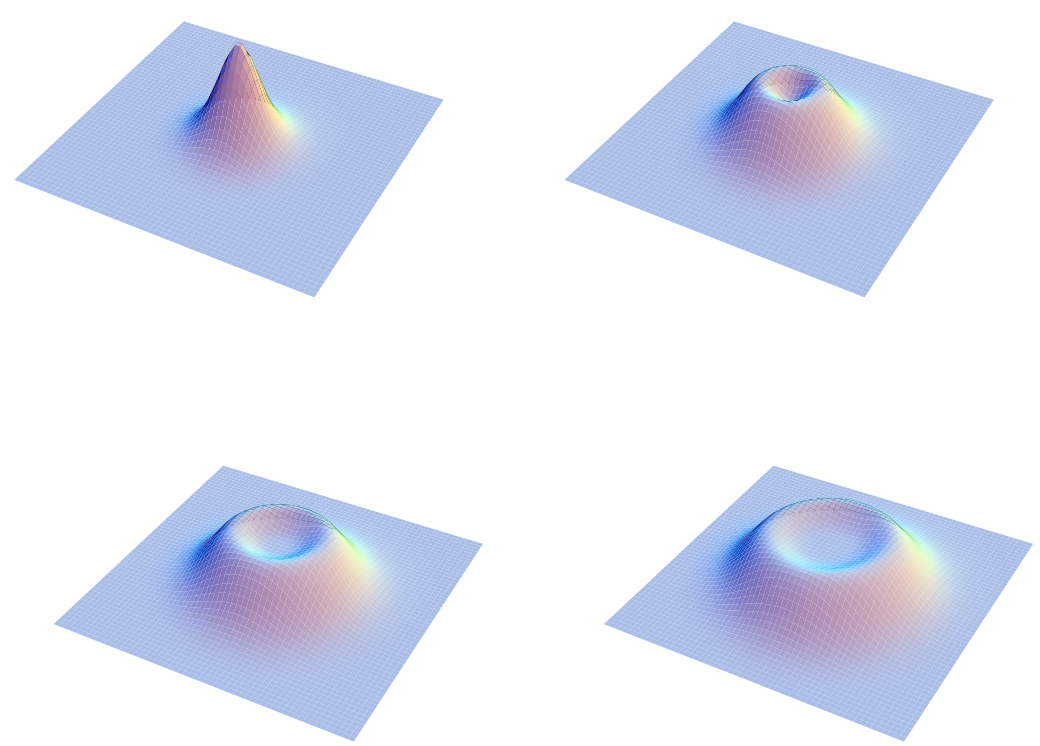

Figure 14: 3D graphics of the energy density for $l=1, l=2, l=3$ and $l=4$ self-dual symmetric ANO vortices.

\begin{tabular}{|c|cc|cc||c|cc|cc|}
\hline & \multicolumn{2}{|c|}{$l=1$} & \multicolumn{2}{c|}{$l=2$} & \multicolumn{2}{c|}{$l=3$} & \multicolumn{2}{c|}{$l=4$} \\
\hline$n$ & $\operatorname{tr} c_{n}(K)$ & $c_{n}\left(K^{G}\right)$ & $\operatorname{tr} c_{n}(K)$ & $c_{n}\left(K^{G}\right)$ & $n$ & $\operatorname{tr} c_{n}(K)$ & $c_{n}\left(K^{G}\right)$ & $\operatorname{tr} c_{n}(K)$ & $c_{n}\left(K^{G}\right)$ \\
\hline 2 & 30.36316 & 2.60773 & 61.06679 & 6.81760 & 2 & 90.20440 & 11.51035 & 118.67540 & 16.46895 \\
3 & 12.94926 & 0.31851 & 25.61572 & 1.34209 & 3 & 36.68235 & 2.60898 & 46.01141 & 4.00762 \\
4 & 4.22814 & 0.022887 & 8.21053 & 0.20481 & 4 & 11.69979 & 0.46721 & 14.64761 & 0.77193 \\
5 & 1.05116 & 0.0011928 & 2.02107 & 0.023714 & 5 & 2.86756 & 0.067279 & 3.58906 & 0.11747 \\
6 & 0.20094 & 0.00008803 & 0.40233 & 0.002212 & 6 & 0.566227 & 0.0079269 & 0.667202 & 0.01620 \\
\hline
\end{tabular}

Table 9: Seeley Coefficients for $l=1, l=2, l=3$ and $l=4$ self-dual symmetric ANO vortices.

\begin{tabular}{|c|cccc|}
\hline$N_{0}$ & $\Delta M_{V}\left(N_{0}\right)$ & $\Delta M_{V}\left(N_{0}\right)$ & $\Delta M_{V}\left(N_{0}\right)$ & $\Delta M_{V}\left(N_{0}\right)$ \\
& $l=1$ & $l=2$ & $l=3$ & $l=4$ \\
\hline 2 & -1.02951 & -2.03787 & -3.01187 & -3.97025 \\
3 & -1.08323 & -2.14111 & -3.15680 & -4.14891 \\
4 & -1.09270 & -2.15913 & -3.18208 & -4.18014 \\
5 & -1.09427 & -2.16212 & -3.18628 & -4.18534 \\
6 & -1.09449 & -2.16257 & -3.18690 & -4.18606 \\
\hline
\end{tabular}

Table 10: One-loop mass shift for $l=1, l=2, l=3$ and $l=4$ self-dual symmetric ANO vortices.

and the last Table provides the one-loop mass shifts of circle symmetric self-dual ANO vortices up to four quanta of magnetic flux taking into account $N_{0}=6$ Seeley coefficients.

\footnotetext{
${ }^{4}$ The Seeley densities corresponding to self-dual ANO vortices superposed at the origin up to four quanta of magnetic flux are given in [ㅁ].
} 


\begin{tabular}{|c|c|}
\hline$l$ & $\Delta M_{V} / \hbar m$ \\
\hline 1 & -1.09449 \\
2 & -2.16257 \\
3 & -3.18690 \\
4 & -4.18606 \\
\hline
\end{tabular}

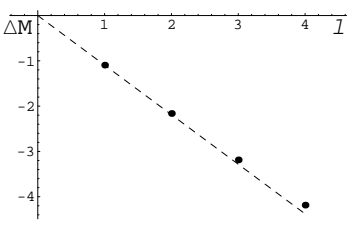

It is remarkable that the one-loop mass shift seems to be linear in $l$. We have found that the oneloop correction to the mass of a circle symmetric vortex of magnetic flux $8 \pi$ is extremely close to four times the one-loop mass shift of a similar vortex of magnetic flux $2 \pi$. This result strongly supports our method: after extremely sophisticated calculations we end with the natural answer.

\section{Prospects on the future of the subject}

We finish by offering a summary of other possible approaches to this subject as well as pointing to other playgrounds where similar methods may work and should be applied.

1. In [49], and [50] Blas and Carrion explored a generalized sine-Gordon model containing as many scalar fields as the rank of the $\mathbb{S L}(N, \mathbb{C})$ Lie group. The potential energy density is determined from the simple roots in such a way that the integrability of the sine-Gordon model is enjoyed by the generalized model. Clearly, there is a correspondence between this model and the ordinary sine-Gordon model that is analogous to the correspondence between the deformed linear $\mathbb{O}(N)$-sigma model and the $\lambda \phi^{4}$ model. There are several types of kinks in this system, which have also been dealt with by the authors in a quantum setting. Nevertheless, it seems to us that the generalized sine-Gordon models offer another excellent arena for applying and improving the quantization method described in this report.

2. In our Lectures we have addressed a massive non-linear $\mathbb{S}^{2}$-sigma model having a rich kink manifold, see [B3]]. In fact, we also computed the one-loop correction to the classical mass of the topological kinks using the Cahill-Comtet-Glauber formula in [B4]. We look forward to attacking this problem by means of the heat kernel/zeta function approach, not only in this system but also in other similar non-linear sigma models where we vary the potential energy density; e.g., adding quartic terms, and/or we change the target space, e.g., to $\mathbb{S}^{3}$.

3. In a very interesting paper [ $[$ ] $]$ Vafa et al. analyzed $\mathscr{N}=2$ supersymmetric LandauGinzburg models that are integrable deformations of the $\mathscr{N}=2$ supersymmetric $A_{k}$ minimal series. The BPS states of these integrable $(1+1)$-dimensional field theories were identified by these authors as "holomorphic" kinks with very noticeable properties. Almost ten years later several of us went through the same set of kinks in [52]. We focused only upon the bosonic sector of the model and chose a real analytic point of view. We believe that the techniques developed in these Lectures provide a procedure for computing the one-loop kink mass shifts due only to bosonic fluctuations. $k^{2}$ is the threshold of the wells and hence we expect to need at least $N_{0}=20$ coefficients in order to reach a good approximation. In the $\mathscr{N}=2$ supersymmetric version of this model, the one-loop correction including bosonic and fermionic fluctuations is zero. Therefore, there is no need to compute the effect of fermionic fluctuations: bosons and fermions created in the kink background cancel each other exactly. 
4. Another strategy may be fruitful. The heat trace, or partition function, which is basic in our approach, admits another conceptual understanding as a path integral over closed world lines:

$$
\begin{aligned}
S_{E}\left[z_{1}, \cdots, z_{N}\right] & =\int_{0}^{1} d \tau\left[\frac{1}{2} \sum_{a=1}^{N} \frac{d z_{a}}{d \tau} \cdot \frac{d z_{a}}{d \tau}+U\left[z_{1}(\tau), \cdots z_{N}(\tau)\right]\right] \\
Z(\beta, l) & =\operatorname{Tr}_{L^{2}} e^{-\beta K}=\Pi_{a=1}^{N} \int_{-\frac{l}{2}}^{\frac{l}{2}} d x_{a} \int_{z_{a}(0)=x_{a}}^{z_{a}(1)=x_{a}} \mathscr{D} z(\tau) e^{-\beta S_{E}\left[z_{1}, \cdots, z_{N}\right]}
\end{aligned}
$$

Here, $\tau=i t$ is imaginary time, $S_{E}$ is the Euclidean action for a particle moving in a cube $\mathbb{I}^{N}$ with varying positions $z_{a}(\tau)$. The path integral is over all the closed paths, and ordinary integration over all the base points of the loops is necessary. It is well known that the Feynman treatment of these path integrals in the high-temperature regime [53] reproduces the heat kernel expansion. What seems more promising is the numerical computation of these world line path integrals using Montecarlo methods, see e.g [54]. Applied to the ANO vortices and semilocal strings this should provide reliable results to be contrasted against our calculations.

5. The Lagrangian density

$$
\mathscr{L}=\frac{1}{2}\left\{\partial_{\mu} \phi \partial^{\mu} \phi-\left[\frac{\lambda_{-}}{l} \delta\left(z+\frac{l}{2}\right)+\frac{\lambda_{+}}{l} \delta\left(z-\frac{l}{2}\right)+4 \sigma^{2}-\frac{6 \sigma^{2}}{\cosh ^{2} \sigma z}\right] \phi^{2}\left(x_{\mu}\right)\right\}
$$

describes the dynamics of a rara avis scalar quantum field theory. The mesons do not move freely even though they do not interact because they are constrained by a background. If $\sigma=0$, the background in (4.2) is formed by two parallel plates located at a distance $l$ from each other in two planes orthogonal to the $z$-axis. The effect of the plates is mimicked by two $\delta$-function potentials of strength $\lambda_{ \pm}$. When $l=\infty$ and $\sigma=1$, the background corresponds to a kink living in the $z$-axis, or to a solitonic/thick domain wall orthogonal to the $z$-axis. Other choices of the couplings provide more complex backgrounds built from these two basic backgrounds.

In Reference [55] Milton wrote the Green's function for the two-plate setup (using Dirichlet boundary conditions). This leads to the energy momentum tensor encoding the Casimir energy -essentially the $T_{00}$ component- and the Casimir force -essentially the $T_{z z}$ component-. It is tempting to perform the same calculation for the kink background. This should provide not only information about the one-loop kink mass shift but should also shed light on the qualitative nature of the forces exerted by the scalar fluctuations on the kink profiles.

6. Over the last year, an interesting paper by Baacke and Kevlishvili was published [56] in which the one-loop shifts to the classical masses of Nielsen-Olesen vortices were obtained by using Green's function methods. The remarkable fact is that the authors gave the quantum corrections with no restriction in the ratio of Higgs and particle masses.

It seems that the time is ready to tackle the problem of the quantum corrections of $Z$ electroweak strings. These topological defects are embedded NO vortices in the neutral $Z_{\mu}$ massive vector field of electroweak theory, and the calculation may will be of experimental interest (even though some imaginary contribution to the energy will arise, because 
electroweak strings are unstable). In fact, there is an interesting paper on this subject, see [57], at the non-physical value of the weak angle equal to zero, and even a status report [58].

7. In a longer perspective, one might think of studying the quantum fluctuations of BPS magnetic monopoles. The work in [5] suggests that the bosonic sector of $\mathscr{N}=2$ supersymmetric Yang-Mills is the right model to look into this problem. Even though van Nieuwenhuizen et al. succeeded in computing the one-loop mass shift to $\mathscr{N}=2$ SUSY monopoles, the difficulties in a purely bosonic framework seem insurmountable. First, the second-order operator governing the fluctuations is (for the $\mathbb{S} U(2)$ group) a $21 \times 21$ matrix-Schrödinger operator in three dimensions. Second, the theory is renormalizable, not super-renormalizable. The coupling constant also receives one-loop divergent contributions that must be canceled by the secod-order Seeley coefficient. So who is afraid of this big bad wolf?

\section{References}

[1] T. Tao, "Why are solitons stable?", Bull. Amer. Math. Soc. 46 (2009) 1, [arXiv:0712.1295]

[2] T. H. R. Skyrme, "Particle states of a quantized meson theory", Proc. Roy. Soc. A262 (1961) 233

[3] R. Dashen, B. Hasslacher and A. Neveu, "Non-perturbative methods and extended hadron models in field theory: 1, semiclassical functional methods; 2, two-dimensional models and extended hadrons; 3, four-dimensional non-Abelian models", Phys. Rev. D10 (1974) 4130.

[4] L. D. Faddeev and V. E. Korepin, "Quantum theory of solitons", Phys. Rept. 42C (1978) 1-87.

[5] K. Cahill, A. Comtet, and R. Glauber, "Mass fomulas for static solitons", Phys. Lett. 64B (1976) 283-285.

[6] S. F. Coleman, "Aspects of symmetry", Cambridge University Press, Cambridge, 1985. Chapter 6: "Classical lumps and their quantum descendants".

[7] A. Alonso-Izquierdo, W. Garcia Fuertes, M. A. Gonzalez Leon, J. Mateos Guilarte, J. M. Muñoz Castañeda, and M. de la Torre Mayado, "Lectures on the mass of topological solitons. Heat kernel/zeta function control of one-loop divergences", [arXiv:hep-th/0611180]

[8] A. Alonso-Izquierdo, W. Garcia Fuertes, M.A.Gonzalez Leon, and J. Mateos Guilarte, "Generalized zeta functions and one-loop corrections to quantum kink masses", Nucl. Phys. B 635 (2002) 525-557, [arXiv:hep-th/0201084]

[9] A. Alonso-Izquierdo, W. Garcia Fuertes, M.A. Gonzalez Leon, and J. Mateos Guilarte, "Semi-classical masses of quantum k-component topological kinks", Nucl. Phys. B 638 (2002)378, [arXiv:hep-th/0205137]

[10] A. Alonso-Izquierdo, W. Garcia Fuertes, M.A. Gonzalez Leon, and J. Mateos Guilarte, "One-loop corrections to classical masses of kink families", Nucl. Phys. B 681 (2004) 163-194, [arXiv:0304125]

[11] A. Rebhan, and P. van Nieuwenhuizen, "No saturation of the quantum Bogomolny bound by two-dimensional $\mathscr{N}=1$ solitons", Nucl. Phys. B508 (1997) 449, [arXiv:hep-th/9707163]

[12] H. Nastase, M. Stephanov, A. Rebhan, and P. van Nieuwenhuizen, "Topological boundary conditions, the BPS bound and elimination of ambiguities in quantum mass of solitons", Nucl. Phys. B542 (1999) 471, [arXiv:hep-th/9802074] 
[13] M. Shifman, A. Vainsthein, and M. Voloshin, "Anomaly and quantum corrections to solitons in two-dimensional theories with minimal supersymmetry", Phys. Rev. D59 (1999) 045016, [arXiv:hep-th/9810068]

[14] N. Graham and R. Jaffe, "Energy, central charge, and the BPS bound for $1+1$ dimensional supersymmetric solitons", Nucl. Phys. B544 (1999) 432-447, [arXiv:hep-th/9808140]

[15] R. Wimmer, "Quantization of supersymmetric solitons", Ph. D Thesis, Wien University (2001), [arXiv:hep-th/0109119]

[16] A. Goldhaber, A. Rebhan, P. van Nieuwenhuizen, and R. Wimmer, "Quantum corrections to mass and central charge of supersymmetric solitons", Phys. Rept. 398C (2004) 179, [arXiv:hep-th/0401152]

[17] Anton Rebhan, Peter van Nieuwenhuizen, Robert Wimmer, "New developments in the quantization of supersymmetric solitons (kinks, vortices and monopoles)", Braz. J. Phys. 34 (2004) 1273-1287, [arXiv:0404223]

[18] D. Bazeia, J. R. Nascimento, R. Ribeiro, and D. Toledo, "Soliton stability in systems of two real scalar fields", J. Phys. A30 (1997) 8157-8166, [arXiv:hep-th/9705224]

[19] M.A. Shifman, and M.B. Voloshin, "Degenerate domain wall solutions in supersymmetric theories", Phys. Rev. D57 (1998) 2590-2598, [arXiv:hep-th/9709137]

[20] A. Alonso-Izquierdo, M.A. Gonzalez Leon, J. Mateos Guilarte, "The kink variety in systems of two coupled scalar fields in two space-time dimensions", Phys. Rev. D65 (2002) 085012, [arXiv:hep-th/0201200]

[21] M. Bordag, A. Goldhaber, P. van Nieuwenhuizen and D. Vassilevich, "Heat kernel and zeta-function regularization for the mass of the SUSY kink", Phys. Rev. D66 (2002) 125014, [arXiv:hep-th/0203066]

[22] A. A. Abrikosov, "On the magnetic properties of superconductors of the second group", Sov. Phys. JETP5, 1174 (1957)

[23] H. Nielsen and P. Olesen, "Vortex line models for dual strings", Nucl.Phys.B61, 45 (1973)

[24] A. Achucarro, and T. Vachaspati, "Semilocal cosmic strings", Phys. Rev. D44 (1991) 3067.

[25] D. Vassilevich, "Quantum corrections to the mass of the supersymmetric vortex", Phys. Rev. D 68 (2003) 045005, [arXiv:hep-th/0304267]

[26] A. Rebhan, P. van Nieuwenhuizen and R. Wimmer, "Nonvanishing quantum corrections to the mass and central charge of the $N=2$ vortex and BPS saturation", Nucl. Phys. B 679 (2004) 382-394; [arXiv:hep-th/0307282]

[27] M. Bordag and I. Drozdov, "Fermionic vacuum energy from a Nielsen-Olesen vortex", Phys. Rev. D68(2003) 065026, [arXiv:hep-th/0305002]

[28] A. Alonso Izquierdo, W. Garcia Fuertes, J. Mateos Guilarte, and M. de la Torre Mayado, "Quantum corrections to the mass of self-dual vortices", Phys. Rev. D70 (2004) 061702(R), [arXiv:hep-th/0406129]

[29] A. Alonso Izquierdo, W. Garcia Fuertes, J. Mateos Guilarte, and M. de la Torre Mayado, "Quantum oscillations of self-dual Abrikosov-Nielsen-Olesen vortices", Phys. Rev. D71 (2005) 125010, [arXiv:hep-th/0504143]

[30] A. Alonso Izquierdo, W. Garcia Fuertes, J. Mateos Guilarte, and M. de la Torre Mayado, “One-loop mass shift formula for kinks and self-dual vortices", Jour. Phys. A39 (2006) 6463-6472, [arXiv:hep-th/0510234] 
[31] A. Alonso Izquierdo, W. Garcia Fuertes, J.M. Guilarte, and M. de la Torre. Mayado, “One loop corrections to the mass of self-dual semi-local planar topological solitons", Nucl.Phys. B797, (2008)431-463, [arXiv:0707.4592]

[32] A. Alonso Izquierdo, W. Garcia Fuertes, J. Mateos Guilarte, and M. de la Torre Mayado, “One-loop fluctuations of semi-local self-dual vortices", J. Phys. A41:164050,2008, [arXiv:0710.3135]

[33] A. Alonso-Izquierdo, M. A. Gonzalez Leon and J. Mateos Guilarte. "Kinks in a non-linear massive sigma model", Physical Review Letters. PRL 101: 131602,2008, [arXiv:0808.3052]

[34] A. Alonso-Izquierdo, M. A. G. Leon and J. M. Guilarte, "BPS and non-BPS kinks in a non-linear $\mathbb{S}^{2}$-massive sigma model", Phys. Rev. D79: 125003, 2009, [arXiv:0903.0593]

[35] E. Elizalde, S. Odintsov, A. Romeo, A Bytsenko, and S. Zerbini, "Zeta regularization techniques with applications", Singapore, World Scientifique, 1994.

[36] K. Kirsten, "Spectral functions in mathematics and physics, Chapman and Hall/CRC, New York, 2002

[37] D. V. Vassilevich, “Heat kernel expansion: user's manual", Phys. Rep. 388C (2003) 279-360, [arXiv:hep-th/0306138]

[38] S. F. Coleman, "There are no Goldstone bosons in two dimensions", Comm. Math. Phys. 31(1973) 259

[39] M. Schlichenmaier, "An introduction to Riemann surfaces, alebraic curves and moduli spaces", Lecture Notes in Physics, Springer Verlag, Berlin 1989

[40] J. Baacke, and N. Kevlishvili, "Self-consistent bounces in two-dimensions", Phys. Rev. D71 (2005) 025008, [arXiv:hep-th/0411162]]

[41] I. G. Avramidi, "Heat kernel approach in quantum field theory", Nucl. Phys. Proc. Supl. 104 (2002) 3-32, [arXiv:hep-th/0107018]

[42] M. Abramowitz and I. Stegun, "Handbook of mathematical functions with formulas, graphs and mathematical tables", Dover, New York, 1992 .

[43] G. Barton, "Levinson's theorem in one dimension: heuristics", Jour. Phys. A18 (1985) 479

[44] L. J. Boya, "Quantum-mechanical scattering in one dimension", Riv. Nuov. Cim. 31 (2008) 75

[45] A. Achucarro, and T. Vachaspati, "Semilocal and electroweak strings", Phys. Rept. 327C (2000) 347, [arXiv:hep-ph/9904229]

[46] G.W. Gibbons, M.E. Ortiz, F. Ruiz Ruiz and T.M. Samols, "Semilocal strings and monopoles", Nucl.Phys. B385 (1992) 127, [arXiv:hep-th/9203023]

[47] H. J. de Vega and F. A. Schaposnik, "A classical vortex solution of the Abelian Higgs model", Phys. Rev. D14 (1976) 1100.

[48] F. Correa, and M. S. Plyushchay, "Hidden supersymmetry in quantum bosonic systems", Ann. Phys. 322:2493-2500,2007,. [hep-th/0605104]

[49] H. Blas, H. L. Carrion, "Solitons, kinks and extended hadron model based on the generalized sine-Gordon theory", JHEP 0701:027,2007, [arXiv:hep-th/0610107]

[50] H. Blas, H. L. Carrion, "Solitons as baryons and qualitons as constituent quarks in two-dimensional QCD", “The Physics of Quarks: New Research", Nova Science Publisher, 2008, [arXiv:0803.0536]

[51] P. Fendley, S. D. Mathur, C. Vafa, and N. P. Warner, “Integrable deformations and scattering matrices for the $\mathscr{N}=2$ supersymmetric discrete series", Phys. Lett. B243, (1990) 257-264 
[52] A. Alonso-Izquierdo, M. A. G. Leon and J. M. Guilarte, "N=2 supersymmetric kinks and real algebraic curves", Phys. Lett. B480 (2000) 373-380, [arXiv:hep-th/0002082]

[53] R. P. Feynman, "Statistical Mechanics", The Benjamin/Cummings Publishing Company, Reading, Massachussets, 1981

[54] H. Gies, K. Langfeld, and L. Moyaerts, "Casimir effect on the world line", JHEP (2003) 0306:018, [arXiv:hep-th/0303264]

[55] K. A. Milton, "The Casimir effect: Recent controversies and progress", J. Phys. A37 (2004) R209, [arXiv:hep-th/0406024]

[56] J. Baacke, N. Kevlishvili, "One-loop corrections to the string tension of the vortex in the Abelian Higgs model”, Phys.Rev. D78 (2008)085008, [arXiv:0806.4349]

[57] N. Graham, M. Quandt, O. Schroeder, and H. Weigel, "Quantum energies of strings in a $(2+1)$-dimensional gauge theory", Nucl. Phys. B758(2006)112-143, [arXiv:hep-th/0607092]

[58] N. Graham, M. Quandt, O. Schroeder, and H. Weigel, “Quantum stabilization of Z-strings, a status report on $D=(3+1)$-dimensions", J. Phys. A41(2008)164099, [arXiv:0710.4386]

[59] A. Rebhan, P. van Nieuwenhuizen, and R. Wimmer, "A new anomalous contribution to the central charge of the $\mathscr{N}=2$ monopole", Phys. Lett. B594 (2004) 234, [arXiv:hep-th/0401116] 